\title{
A mixed formulation of the Stefan problem with surface tension
}

\author{
CHRistopher B. DAVIS \\ Department of Mathematics, Tennessee Tech University, \\ 1 William L Jones Dr, Cookeville, TN 38505, U.S.A. \\ E-mail: cbdavis@tntech.edu \\ SHAWN W. WALKER \\ Department of Mathematics, Louisiana State University, Baton Rouge, LA 70803-4918, U.S.A. \\ E-mail:walker@math.lsu.edu
}

[Received 20 December 2013 and in revised form 16 January 2015]

\begin{abstract}
A dual formulation and finite element method is proposed and analyzed for simulating the Stefan problem with surface tension. The method uses a mixed form of the heat equation in the solid and liquid (bulk) domains, and imposes a weak formulation of the interface motion law (on the solidliquid interface) as a constraint. The basic unknowns are the heat fluxes and temperatures in the bulk, and the velocity and temperature on the interface. The formulation, as well as its discretization, is viewed as a saddle point system. Well-posedness of the time semi-discrete and fully discrete formulations is proved in three dimensions, as well as an a priori (stability) bound and conservation law. Simulations of interface growth (in two dimensions) are presented to illustrate the method.
\end{abstract}

2010 Mathematics Subject Classification: Primary 65M60; Secondary 35K20

Keywords: Stefan problem; mixed method; energy stability; interface motion; semi-implicit scheme.

\section{Introduction}

\section{$1.1 \quad$ Background}

The Stefan problem describes the geometric evolution of a solidifying (or melting) interface. It is a classic problem in phase transitions. The model consists of time-dependent heat diffusion in the solid and liquid phases, with an interfacial condition on the solid-liquid interface known as the Gibbs-Thomson relation with kinetic undercooling [41, 42, 61]. A thermodynamic derivation of the model can be found in [29]. Applications range from modeling the freezing (or melting) of water to the solidification of crystals from a melt and dendritic growth [15, 30, 38, 51, 52, 59]. Mathematical theory for the Stefan problem with Gibbs-Thomson law is available for local and global in time solutions [13, 25, 36, 39, 45-48]. Well-posedness results are also available if the heat equation in the bulk phases is replaced by a quasi-static approximation (i.e. the Mullins-Sekerka problem) [18, 20, 24, 40, 49].

Efficient numerical schemes for simulating these models is necessary to allow for design, prediction, and optimization of these processes. Phase-field methods have been used for simulating solidification and dendrite growth $[6,35,55]$. Level set methods have also been used to handle the evolutions of the two phase interface $[12,23,44,54]$. The method we present uses a fronttracking approach where the interface parametrization conforms to a surrounding bulk mesh. Other front-tracking methods for the Stefan problem have also been given [2, 4, 34, 35, 50-53]. 
Our paper presents a completely mixed formulation of the Stefan problem, including the bulk heat equations [8]. In other words, we formulate the problem in a saddle-point framework, where the heat equations are in mixed form, and the interface motion law appears as a constraint in the system of equations with a balancing Lagrange multiplier that represents the interface temperature. To the best of our knowledge, this is a new method for the Stefan problem with surface tension. Some highlights of our method are the following.

- We prove that both the time semi-discrete and fully discrete systems have a priori bounds (in time) that mimic the continuous model. This assumes the interface velocity is reasonably regular and that there are no topological changes. Moreover, we can prove that both the time semi-discrete and fully discrete systems maintain conservation of thermal energy. In [5], they only achieve this for their discrete in space, continuous in time, scheme.

- The interface is represented by a surface triangulation that conforms to the bulk mesh which deforms with the interface. Hence, occasional re-meshing is needed, which is done by the method in [63]. One advantage of this method is that all integrals in the finite element formulation can be computed exactly. In addition, we do not need to compute the intersection of meshes at adjacent time steps to transfer solution variables from one mesh to the next (e.g. for computing $L^{2}$ projections from one mesh to another).

- Our method can be modified to include anisotropic surface tension via [5], which is relevant to crystal growth. The well-posedness of the method remains unchanged, as well as the a priori bound and conservation law.

- Other variations of the Stefan problem (e.g. Mullins-Sekerka) can be formulated with our approach by straightforward modifications. One can even include moving contact line effects when the solid phase is attached to a rigid boundary [60, 64].

\subsection{Summary}

In Section 2, we describe the governing equations. Section 3 describes the fully continuous weak formulation and derives a formal a priori bound and conservation law. Section 4 explains the timediscretization and how the interface motion is handled. A variational formulation of the time semidiscrete problem is given, its well-posedness is shown, and an a priori bound and conservation law is proved. We then do the same for the fully-discrete formulation (Section 5). Section 6 concludes with numerical simulations to demonstrate the method.

\section{Model for the Stefan problem with surface tension}

The particular mathematical model we consider can be found in [5, 29]. In this section, we present the strong form of the Stefan problem.

\subsection{Notation}

Let $\Omega$ be a fixed domain in $\mathbb{R}^{d}$ (for $d=2,3$ ), with outer boundary $\partial \Omega$, that contains two phases, liquid and solid, denoted respectively by the open sets $\Omega_{1}$ and $\Omega_{\mathrm{s}}$, i.e. $\Omega=\operatorname{int}\left(\overline{\Omega_{1}} \cup \overline{\Omega_{\mathrm{s}}}\right)$ and $\Omega_{1} \cap \Omega_{\mathrm{s}}=\emptyset$ (see Figure 1). Furthermore, $\partial \Omega$ partitions into two pieces: $\partial \Omega=\overline{\partial_{\mathrm{D}} \Omega} \cup \overline{\partial_{\mathrm{N}} \Omega}$ such that $\partial_{\mathrm{D}} \Omega \cap \partial_{\mathrm{N}} \Omega=\emptyset$ and $\left|\partial_{\mathrm{D}} \Omega\right|>0$ (set of positive measure).

The solid-liquid interface between the phases is $\Gamma=\overline{\Omega_{1}} \cap \overline{\Omega_{\mathrm{s}}}$ (a closed surface). The domains $\Omega_{1}, \Omega_{\mathrm{s}}$, and $\Gamma$ are time-dependent, and we assume that $\Gamma(t) \subset \Omega$ for all $t$. Moreover, in order to 
write the strong form of the Stefan problem (Section 2.2), we assume $\Gamma(t)$ is smooth and let $\mathbf{X}(t)$ denote a parametrization of $\Gamma(t)$ :

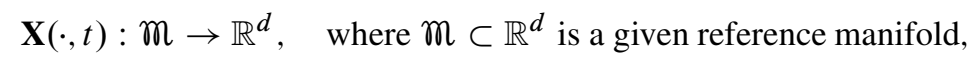

i.e. $\Gamma(t)=\mathbf{X}(m, t)$. Furthermore, we introduce fixed reference domains $\widehat{\Omega}_{1}, \widehat{\Omega}_{\mathrm{s}}$ for the liquid and

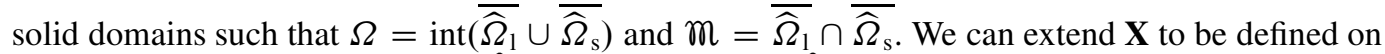
all of $\Omega$ and such that $\Omega_{1}(t)=\mathbf{X}\left(\widehat{\Omega}_{1}, t\right)$ and $\Omega_{\mathrm{s}}(t)=\mathbf{X}\left(\widehat{\Omega}_{\mathrm{s}}, t\right)$ (slight abuse of notation here). This is needed later when specifying the function spaces.

The surface $\Gamma$ has a unit normal vector $\nu$ that is assumed to point into $\Omega_{1}$ (see Figure 1). For quantities $q$ in $\Omega_{1}\left(\Omega_{\mathrm{s}}\right)$, we append a subscript: $q_{1}\left(q_{\mathrm{s}}\right)$. The symbol $\kappa$ represents the summed curvature of the interface $\Gamma$ (sum of the principle curvatures), and we assume the convention that $\kappa$ is positive when $\Omega_{\mathrm{s}}$ is convex (contrary to [5]).

Table 1 summarizes the notation we use for the physical domain and the physical variables (e.g. temperature, etc.). The physical coefficient symbols that appear in the model, as well as their values, are given in Table 2. The non-dimensional parameters are given in Table 3.
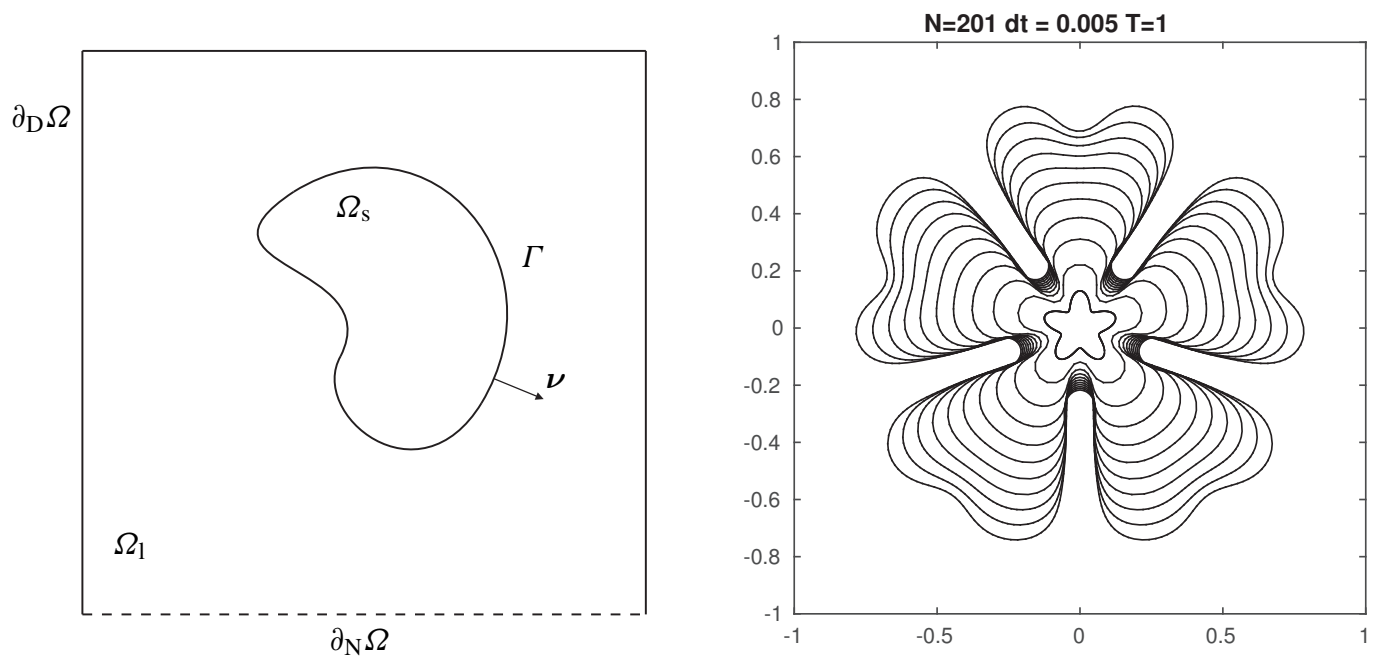

FIG. 1: Left: Domains in the Stefan problem. The entire "box" is $\Omega=\operatorname{int}\left(\overline{\Omega_{1}} \cup \overline{\Omega_{\mathrm{s}}}\right.$ ) (containing two phases $\Omega_{1}, \Omega_{\mathrm{s}}$ ) with Dirichlet boundary $\partial_{\mathrm{D}} \Omega$ denoted by the dashed line. A Neumann condition is applied on the remaining sides $\partial_{\mathrm{N}} \Omega$. The interface between the phases is $\Gamma=\overline{\Omega_{1}} \cap \overline{\Omega_{\mathrm{s}}}$ with unit normal vector $\boldsymbol{\nu}$ pointing into $\Omega_{1}$. Right: Simulation using the method developed in this paper (isotropic surface tension). Several time-lapses are shown to illustrate the evolution with initial interface having a "star" shape. See Section 6 for more simulations. 
TABLE 1: General notation and symbols

\begin{tabular}{lll}
\hline Symbol & Name & Units \\
\hline$\Omega, \Omega_{1}, \Omega_{\mathrm{s}}$ & Bulk Domains: Entire, Liquid, Solid & - \\
$\partial \Omega$ & Boundary of $\Omega$ & - \\
$\partial_{\mathrm{D}} \Omega, \partial_{\mathrm{N}} \Omega$ & Partition of $\partial \Omega=\overline{\partial_{\mathrm{D}} \Omega} \cup \overline{\partial_{\mathrm{N}} \Omega}$ & - \\
$\Gamma$ & Interface between $\Omega_{1}$ and $\Omega_{\mathrm{s}}$ phases & - \\
$\mathbf{X}, \mathbf{V}$ & Interface $(\Gamma)$ Parametrization and Velocity & $\mathrm{m}, \mathrm{m} \mathrm{s}$ \\
$u_{1}, u_{\mathrm{s}}$ & Temperature in $\Omega_{1}$ and $\Omega_{\mathrm{s}}$ & $\mathrm{K}(\mathrm{deg}$. Kelvin) \\
$f_{1}, f_{\mathrm{s}}$ & Heat sources in $\Omega_{1}$ and $\Omega_{\mathrm{s}}$ & $\mathrm{J} \mathrm{m} \mathrm{s}^{-1}$ \\
$\nabla_{\Gamma}, \Delta_{\Gamma}$ & Surface Gradient and Laplace-Beltrami Opera- & $\mathrm{m}^{-1}, \mathrm{~m}^{-2}$ \\
$\nu$ & tor & \\
$\nabla_{\Gamma} \mathbf{X}:=\mathbf{I}-\boldsymbol{\nu} \otimes \boldsymbol{\nu}$ & Unit Normal Vector of $\Gamma$ & - \\
$\kappa, \kappa \boldsymbol{\nu}:=-\Delta_{\Gamma} \mathbf{X}$ & Projection onto Tangent Space of $\Gamma$ & - \\
\hline
\end{tabular}

TABLe 2: Physical parameters and values

\begin{tabular}{lll}
\hline Symbol & Name & Units \\
\hline$\vartheta$ & Volumetric Heat Capacity & $\mathrm{J} \mathrm{m}^{-3} \mathrm{~K}^{-1}$ \\
$K_{1}, K_{\mathrm{S}}$ & Thermal Conductivity in $\Omega_{1}$ and $\Omega_{\mathrm{s}}$ & $\mathrm{J} \mathrm{s}^{-1} \mathrm{~m}^{-1} \mathrm{~K}^{-1}$ \\
$L$ & Latent Heat Coefficient & $\mathrm{J} \mathrm{m}^{-3}$ \\
$\alpha$ & Surface Tension Coefficient of $\Gamma$ & $\mathrm{J} \mathrm{m}^{-2}$ \\
$S$ & Volumetric Entropy Coefficient & $\mathrm{J} \mathrm{m}^{-3} \mathrm{~K}^{-1}$ \\
$\rho$ & Kinetic Coefficient & $\mathrm{J} \mathrm{s} \mathrm{m}$ \\
$\beta$ & Mobility Coefficient & - \\
$D$ & Length Scale & $\mathrm{m}$ \\
$U_{0}=T_{\mathrm{M}}$ & Temperature Scale & $\mathrm{K}$ \\
$t_{0}$ & Time Scale & $\mathrm{seconds}(\mathrm{s})$ \\
$F_{0}=\vartheta U_{0} / t_{0}$ & Heat Source Scale & $\mathrm{J} \mathrm{m}^{-3} \mathrm{~s}^{-1}$ \\
\hline
\end{tabular}

TABLE 3: Nondimensional parameters

\begin{tabular}{lll}
\hline Symbol & Name & Value \\
\hline$\widehat{S}=S / \vartheta$ & entropy coefficient & 2 \\
$\widehat{\beta}_{0}=\vartheta U_{0} t_{0} /(\rho D)$ & mobility coefficient & 0.01 \\
$\widehat{\beta}=\widehat{\beta}_{0} \beta$ & mobility function & - \\
$\widehat{K}_{1}=K_{1} t_{0} /\left(D^{2} \vartheta\right)$ & liquid conductivity & 1 \\
$\widehat{K}_{\mathrm{s}}=K_{\mathrm{s}} t_{0} /\left(D^{2} \vartheta\right)$ & solid conductivity & 1 \\
$\widehat{\alpha}=\alpha /\left(U_{0} D \vartheta\right)$ & surface tension coefficient & 0.0005 \\
\hline
\end{tabular}




\subsection{Strong formulation}

The Stefan problem is as follows. Find $u: \Omega \times[0, T] \rightarrow \mathbb{R}$ and interface $\Gamma(t) \subset \Omega$ for all $t \in(0, T]$, such that $\left.u\right|_{\Omega_{1}}=u_{1},\left.u\right|_{\Omega_{\mathrm{s}}}=u_{\mathrm{s}}$, and the following bulk conditions hold:

$$
\begin{aligned}
\vartheta \partial_{t} u_{1}-K_{1} \Delta u_{1} & =f_{1}, \text { in } \Omega_{1}(t), \\
\vartheta \partial_{t} u_{\mathrm{s}}-K_{\mathrm{s}} \Delta u_{\mathrm{s}} & =f_{\mathrm{s}}, \text { in } \Omega_{\mathrm{s}}(t), \\
\nu_{\Omega} \cdot \nabla u & =0, \text { on } \partial_{\mathrm{N}} \Omega, \\
u & =u_{\mathrm{D}}, \text { on } \partial_{\mathrm{D}} \Omega, \\
u(\cdot, 0) & =u_{0}, \text { in } \Omega,
\end{aligned}
$$

where $u_{0}$ is the initial temperature, and the following interface conditions hold:

$$
\begin{aligned}
u_{1}-u_{\mathrm{s}} & =0, \text { on } \Gamma(t), \\
\nu \cdot\left(K_{1} \nabla u_{1}-K_{\mathrm{s}} \nabla u_{\mathrm{s}}\right)+L \partial_{t} \mathbf{X} \cdot \boldsymbol{\nu} & =0, \text { on } \Gamma(t), \\
\frac{\rho}{\beta(\nu)} \partial_{t} \mathbf{X} \cdot \nu+\alpha \kappa+S u & =0, \text { on } \Gamma(t), \\
\mathbf{X}(\cdot, 0)-\mathbf{X}_{0}(\cdot) & =\mathbf{0}, \text { on } m, \\
\Gamma(0) & =\Gamma_{0}, \text { in } \Omega,
\end{aligned}
$$

where $\Gamma_{0}$ is the initial interface (parameterized by $\mathbf{X}_{0}$ ) and $\mathbf{X}(\cdot, t)$ parameterizes $\Gamma(t)$. Note that $u=T-T_{\mathrm{M}}$, where $T$ is the temperature in degrees Kelvin and $T_{\mathrm{M}}$ is the melting temperature at the interface $\Gamma$, and that $u$ is continuous across the interface. As noted in [5], we must have

$$
S=\frac{L}{T_{\mathrm{M}}}
$$

\subsection{Non-dimensionalization}

We non-dimensionalize the variables, but use the same variable symbols for convenience. This gives

$$
\begin{aligned}
\partial_{t} u_{1}-\widehat{K}_{1} \Delta u_{1} & =f_{1}, \text { in } \Omega_{1}(t), \\
\partial_{t} u_{\mathrm{s}}-\widehat{K}_{\mathrm{s}} \Delta u_{\mathrm{s}} & =f_{\mathrm{s}}, \text { in } \Omega_{\mathrm{s}}(t), \\
\nu_{\Omega} \cdot \nabla u & =0, \text { on } \partial_{\mathrm{N}} \Omega, \\
u & =u_{\mathrm{D}}, \text { on } \partial_{\mathrm{D}} \Omega, \\
u(\cdot, 0) & =u_{0}, \text { in } \Omega, \\
u_{1}-u_{\mathrm{s}} & =0, \text { on } \Gamma(t), \\
\nu \cdot\left(\widehat{K}_{1} \nabla u_{1}-\widehat{K_{\mathrm{s}}} \nabla u_{\mathrm{s}}\right)+\widehat{S} \partial_{t} \mathbf{X} \cdot \nu & =0, \text { on } \Gamma(t), \\
\frac{1}{\widehat{\beta}(\nu)} \partial_{t} \mathbf{X} \cdot \nu+\widehat{\alpha} \kappa+\widehat{S} u & =0, \text { on } \Gamma(t), \\
\mathbf{X}(\cdot, 0)-\mathbf{X}_{0}(\cdot) & =\mathbf{0}, \text { on } m, \\
\Gamma(0) & =\Gamma_{0}, \text { in } \Omega .
\end{aligned}
$$


Throughout the paper, we assume the non-dimensional coefficients satisfy

$$
\infty>\widehat{K}_{1}, \widehat{K}_{\mathrm{s}}, \widehat{\alpha}, \widehat{S}>0, \quad \infty \geqslant \widehat{\beta}(\boldsymbol{\nu}) \geqslant \widehat{\beta}_{-}>0, \quad \text { where } \widehat{\beta}_{-} \text {is a constant. }
$$

REMARK 2.1 The case of $\vartheta=0$ (i.e. $\widehat{\alpha}, \widehat{S}, \widehat{K_{1}}, \widehat{K}_{\mathrm{s}}=\infty$ ) corresponds to the steady-state heat equation in $\Omega_{1}$ and $\Omega_{\mathrm{s}}$ and if $\rho=0$ (i.e. $\left.\widehat{\beta}(\boldsymbol{\nu}) \equiv \infty\right)$ ) then (2.5) and (2.6) becomes the Mullins-Sekerka problem with Gibbs-Thomson law [41]. Our formulation can easily be modified to implement this model. If $\widehat{S} \equiv \infty$ only, then $\partial_{t} \mathbf{X} \cdot \boldsymbol{\nu} \equiv 0$, so (2.5) and (2.6) reduce to the time-dependent heat equation on a stationary domain with $u_{1}=u_{\mathrm{s}}=0$ on $\Gamma$.

\section{Weak formulation}

\subsection{Function spaces}

Since the domain and interface deform in time, we define the function spaces using a reference domain [5]. For simplicity, we shall assume that $\partial \Omega \cap \partial \Omega_{1}=\partial \Omega$ (see Figure 1); thus, $\overline{\Omega_{\mathrm{s}}} \subset \Omega$. We use standard notation for denoting Sobolev spaces [1, 57], e.g. $L^{2}(\Omega)$ is the space of square integrable functions on $\Omega$. For any vector-valued function $\eta$, if we write $\boldsymbol{\eta} \in L^{2}(\Omega)$, we mean each component of $\boldsymbol{\eta}$ is in $L^{2}(\Omega)$. Continuing, we have $H^{1}(\Omega)=\left\{u \in L^{2}(\Omega): \nabla u \in L^{2}(\Omega)\right\}$ and $H(\operatorname{div}, \Omega)=\left\{\boldsymbol{\eta} \in L^{2}(\Omega): \nabla \cdot \eta \in L^{2}(\Omega)\right\}$. The norms on these spaces are defined in the obvious way, i.e. $\|u\|_{L^{2}(\Omega)}^{2}=\int_{\Omega}|u|^{2},\|u\|_{H^{1}(\Omega)}^{2}=\|u\|_{L^{2}(\Omega)}^{2}+\|\nabla u\|_{L^{2}(\Omega)}^{2},\|\boldsymbol{\eta}\|_{H(\operatorname{div}, \Omega)}^{2}=$ $\|\boldsymbol{\eta}\|_{L^{2}(\Omega)}^{2}+\|\nabla \cdot \boldsymbol{\eta}\|_{L^{2}(\Omega)}^{2}$.

For a general function $f: \Omega \rightarrow \mathbb{R}$, we denote its trace (or restriction) to a sub-domain $\Sigma \subset \Omega$ (of co-dimension 1) by $\left.f\right|_{\Sigma}$. The trace of a function in $H^{1}(\Omega)$ is well-defined; for a function in $L^{2}(\Omega)$, the trace is not well-defined. Moreover, the trace of all functions (on $\Sigma \subset \Omega$ ) in $H^{1}(\Omega)$ spans a Hilbert space, denoted $H^{1 / 2}(\Sigma)$, which is a proper dense subspace of $L^{2}(\Sigma)$. Referring to [7, pg. 48], the norm for $H^{1 / 2}(\partial \Omega)$ is defined by

$$
\|v\|_{H^{1 / 2}(\partial \Omega)}:=\inf _{\substack{\left.w \in H^{1}(\Omega) \\ w\right|_{\partial \Omega}=v}}\|w\|_{H^{1}(\Omega)}=\|\bar{v}\|_{H^{1}(\Omega)},
$$

where $\bar{v}$ is the unique weak solution of $-\Delta \bar{v}+\bar{v}=0$ in $\Omega$, with $\bar{v}=v$ on $\partial \Omega$. We also have $H^{-1 / 2}(\partial \Omega)$, i.e. the dual space of $H^{1 / 2}(\partial \Omega)$ with the dual norm,

$$
\|\varrho\|_{H^{-1 / 2}(\partial \Omega)}:=\sup _{v \in H^{1 / 2}(\partial \Omega)} \frac{\langle\varrho, v\rangle_{\partial \Omega}}{\|v\|_{H^{1 / 2}(\partial \Omega)}},
$$

where $\langle\cdot, \cdot\rangle_{\partial \Omega}$ denotes the duality pairing between $H^{-1 / 2}(\partial \Omega)$ and $H^{1 / 2}(\partial \Omega)$.

It is well known [26, Theorem 1.7], [7, Lemma 2.1.1] that $\left.\boldsymbol{\eta} \cdot \boldsymbol{\nu}\right|_{\partial \Omega}$ is in $H^{-1 / 2}(\partial \Omega)$ for all $\boldsymbol{\eta}$ in $H(\operatorname{div}, \Omega)\left(\nu_{\Omega}\right.$ is the unit normal vector on $\left.\partial \Omega\right)$. In fact, by [26, (1.44)], we have that

$$
\|\boldsymbol{\eta} \cdot \boldsymbol{\nu}\|_{H^{-1 / 2}(\partial \Omega)} \leqslant\|\boldsymbol{\eta}\|_{H(\operatorname{div}, \Omega)} \text {, for all } \boldsymbol{\eta} \in H(\operatorname{div}, \Omega) \text {. }
$$

With this, one can show that $\|v\|_{H^{1 / 2}(\partial \Omega)}$ has a dual norm realization.

PROPOSITION 3.1

$$
\|v\|_{H^{1 / 2}(\partial \Omega)}=\sup _{\boldsymbol{\eta} \in H(\operatorname{div}, \Omega)} \frac{\langle\boldsymbol{\eta} \cdot \boldsymbol{\nu}, v\rangle_{\partial \Omega}}{\|\boldsymbol{\eta}\|_{H(\mathrm{div}, \Omega)}} .
$$


Enforcing boundary conditions requires the trace. To this end, let $H_{0, D}^{1}(\Omega)=\left\{u \in H^{1}(\Omega)\right.$ : $\left.u\right|_{\partial_{\mathrm{D}} \Omega}=0$ \}. On the reference domains $\widehat{\Omega}_{1}$ and $\widehat{\Omega}_{\mathrm{s}}$, we introduce:

$$
\begin{aligned}
\mathbb{V} & =H(\operatorname{div}, \Omega), \quad \mathbb{V}(g)=\left\{\boldsymbol{\eta} \in \mathbb{V}:\left\langle\boldsymbol{\eta} \cdot \boldsymbol{\nu}_{\Omega}-g, q\right\rangle_{\partial \Omega}=0, \forall q \in H_{0, D}^{1}(\Omega)\right\}, \\
\mathbb{V}_{1}=H\left(\operatorname{div}, \widehat{\Omega}_{1}\right), & \mathbb{V}_{1}(g)=\left\{\boldsymbol{\eta} \in \mathbb{V}_{1}:\left\langle\boldsymbol{\eta} \cdot \boldsymbol{\nu}_{\Omega}-g, q\right\rangle_{\partial \Omega}=0, \forall q \in H_{0, D}^{1}(\Omega)\right\}, \\
\mathbb{V}_{\mathrm{s}}=H\left(\operatorname{div}, \widehat{\Omega}_{\mathrm{s}}\right), &
\end{aligned}
$$

where $g$ is in $H^{-1 / 2}(\partial \Omega)$ (see [7, Remark 2.1.3]). We also have the spaces

$$
\mathbb{Q}=L^{2}(\Omega), \quad \mathbb{Q}_{1}=L^{2}\left(\widehat{\Omega}_{1}\right), \quad \mathbb{Q}_{\mathrm{s}}=L^{2}\left(\widehat{\Omega}_{\mathrm{s}}\right) .
$$

On the reference manifold $m$, we define [1]

$$
\mathbb{M}=H^{1 / 2}(m, \mathbb{R}), \quad \mathbb{Y}=H^{1}\left(m, \mathbb{R}^{d}\right) .
$$

The norm for $\mathbb{Y}$ is $\|\mathbf{V}\|_{H^{1}(\Gamma)}^{2}=\int_{\Gamma}|\mathbf{V}|^{2}+\int_{\Gamma}\left|\nabla_{\Gamma} \mathbf{V}\right|^{2}$ (see Section 3.2.1 for $\nabla_{\Gamma}$ ).

We use the following abuse of notation, similar to [5]. We identify functions $\eta_{1}$ in $\mathbb{V}_{1}$ with $\eta_{1} \circ \mathbf{X}^{-1}$ defined on $\Omega_{1}(t)$ (recall $\Omega_{1}(t)=\mathbf{X}\left(\widehat{\Omega}_{1}, t\right)$ ), and denote both functions simply as $\eta_{1}$; similar considerations are made for functions $\boldsymbol{\eta}_{\mathrm{s}}$ in $\mathbb{V}_{\mathrm{s}}$. Likewise, we identify $\mathbf{V}$ in $\mathbb{Y}$ with $\mathbf{V} \circ \mathbf{X}^{-1}$ defined on $\Gamma(t)$, and denote both functions as $\mathbf{V}$; similar considerations are made for functions $\mu$ in $\mathbb{M}$. Along these lines, we have $\mathbb{V}_{1} \simeq H\left(\operatorname{div}, \Omega_{1}\right), \mathbb{V}_{\mathrm{s}} \simeq H\left(\operatorname{div}, \Omega_{\mathrm{s}}\right), \mathbb{Q}_{1} \simeq L^{2}\left(\Omega_{1}\right), \mathbb{Q}_{\mathrm{s}} \simeq L^{2}\left(\Omega_{\mathrm{s}}\right)$, $\mathbb{M} \simeq H^{1 / 2}(\Gamma), \mathbb{Y} \simeq H^{1}(\Gamma)$, provided the mapping $\mathbf{X}$ is not degenerate.

For technical reasons, we need two versions of the $H^{1 / 2}(\Gamma)$ norm related to $\Omega_{1}$ and $\Omega_{\mathrm{s}}$. Define

$$
\|v\|_{H_{1}^{1 / 2}(\Gamma)}:=\sup _{\eta_{1} \in \mathbb{V}_{1}(0)} \frac{\left\langle\boldsymbol{\eta}_{1} \cdot \boldsymbol{\nu}, v\right\rangle_{\Gamma}}{\left\|\boldsymbol{\eta}_{1}\right\|_{H\left(\operatorname{div}, \Omega_{1}\right)}}, \quad\|v\|_{H_{\mathrm{s}}^{1 / 2}(\Gamma)}:=\sup _{\boldsymbol{\eta}_{\mathrm{s}} \in \mathbb{V}_{\mathrm{s}}} \frac{\left\langle\boldsymbol{\eta}_{\mathrm{s}} \cdot \boldsymbol{\nu}, v\right\rangle_{\Gamma}}{\left\|\boldsymbol{\eta}_{\mathrm{s}}\right\|_{H\left(\mathrm{div}, \Omega_{\mathrm{s}}\right)}} .
$$

Basically, these norms are related to the "side" of $\Gamma$ on which we take the trace. We also define the $H^{1 / 2}$ and $H^{-1 / 2}$ norm on $\Gamma$ by

$$
\|v\|_{H^{1 / 2}(\Gamma)}:=\frac{1}{2}\left(\|v\|_{H_{1}^{1 / 2}(\Gamma)}+\|v\|_{H_{\mathrm{s}}^{1 / 2}(\Gamma)}\right), \quad\|\varrho\|_{H^{-1 / 2}(\Gamma)}:=\sup _{v \in H^{1 / 2}(\Gamma)} \frac{\langle\varrho, v\rangle_{\Gamma}}{\|v\|_{H^{1 / 2}(\Gamma)}}
$$

To conclude this section, we define the dual norm for $H^{-1}(\Gamma)$ :

$$
\|\varrho\|_{H^{-1}(\Gamma)}:=\sup _{v \in H^{1}(\Gamma)} \frac{\langle\varrho, v\rangle_{\Gamma}}{\|v\|_{H^{1}(\Gamma)}},
$$

where $\langle\varrho, v\rangle_{\Gamma}$ is understood to be the duality pairing between $H^{-1}(\Gamma)$ and $H^{1}(\Gamma)$.

\subsection{Curvature}

3.2.1 Differential geometry. First, we review some differential geometry [17, 37]. Let $\boldsymbol{\Psi}: U \rightarrow$ $\Gamma$ be a local parameterization of $\Gamma \subset \mathbb{R}^{3}$ where $U \subset \mathbb{R}^{2}$ with local variables $\left(s_{1}, s_{2}\right)$. The first 
fundamental form $g: U \rightarrow \mathbb{R}^{2 \times 2}$ is given by $g_{i j}=\partial_{s_{i}} \boldsymbol{\Psi} \cdot \partial_{s_{j}} \boldsymbol{\Psi}$ for $1 \leqslant i, j \leqslant 2$. Then the tangential gradient (or surface gradient) of $\omega: \Gamma \rightarrow \mathbb{R}$ is defined as

$$
\left(\nabla_{\Gamma} \omega\right) \circ \boldsymbol{\Psi}:=\sum_{i, j=1}^{2} g^{i j} \partial_{s_{i}}(\omega \circ \boldsymbol{\Psi}) \partial_{s_{j}} \boldsymbol{\Psi},
$$

where $\left[g^{i j}\right]_{i, j=1}^{2}=g^{-1}$ (matrix inverse). Given $\mathbf{Y}: \Gamma \rightarrow \mathbb{R}^{3}$, we have $\nabla_{\Gamma} \mathbf{Y}=$ $\left(\nabla_{\Gamma} Y_{1}, \nabla_{\Gamma} Y_{2}, \nabla_{\Gamma} Y_{3}\right)($ a $3 \times 3$ matrix $)$. Moreover, we have the tangential divergence $\nabla_{\Gamma} \cdot \mathbf{Y}:=$ $\operatorname{trace}\left(\nabla_{\Gamma} \mathbf{Y}\right)$.

The Laplace-Beltrami operator is defined by $\Delta_{\Gamma} \omega=\nabla_{\Gamma} \cdot \nabla_{\Gamma} \omega$ which expands out to

$$
\left(\Delta_{\Gamma} \omega\right) \circ \boldsymbol{\Psi}:=\sum_{i, j=1}^{2} g^{i j} \partial_{s_{i}}\left(\sum_{r, k=1}^{2} g^{r k} \partial_{s_{r}}(\omega \circ \boldsymbol{\Psi}) \partial_{s_{k}} \boldsymbol{\Psi}\right) \cdot \partial_{s_{j}} \boldsymbol{\Psi} .
$$

Note: When $\Gamma$ is a one-dimensional curve with oriented unit tangent vector $\tau$, we have $\nabla_{\Gamma} \equiv \tau \partial_{s}$ and $\Delta_{\Gamma} \equiv \partial_{s}^{2}$, where $\partial_{s}$ is the derivative with respect to arc-length.

Therefore, taking $\mathbf{X}(\cdot, t)$ to be a local parameterization of $\Gamma(t)$, the vector curvature $\kappa \boldsymbol{\nu}$ of $\Gamma(t)[17,37]$ is given by $-\Delta_{\Gamma} \mathbf{X}=\kappa \nu$, where $\kappa$ is the sum of the principle curvatures.

3.2.2 Weak form. In the rest of the paper, we take advantage of a weak formulation of the vector curvature [3, 19]. If $\Gamma$ is a closed $C^{2}$ manifold, then the following integration by parts relation is true:

$$
\int_{\Gamma} \kappa \nu \cdot \mathbf{Y}=\int_{\Gamma} \nabla_{\Gamma} \mathbf{X}: \nabla_{\Gamma} \mathbf{Y}, \quad \text { for all } \mathbf{Y} \text { in } \mathbb{Y},
$$

where $\nabla_{\Gamma} \mathbf{X}$ is a symmetric matrix that represents the projection operator onto the tangent space of $\Gamma$, i.e. $\nabla_{\Gamma} \mathbf{X}=\mathbf{I}-\boldsymbol{\nu} \otimes \boldsymbol{\nu}$. We use (3.10) to derive the weak form (3.12).

\subsection{Fully continuous}

We present a mixed formulation of (2.5), (2.6) that is partly related to [8] for the heat equation. Define the flux variables $\sigma_{1}=-\widehat{K}_{1} \nabla u_{1}, \sigma_{\mathrm{s}}=-\widehat{K}_{\mathrm{s}} \nabla u_{\mathrm{s}}$, and take $u_{\mathrm{D}}(\cdot, t)$ in $H_{0, N}^{1}(\Omega)=\{u \in$ $\left.H^{1}(\Omega):\left.u\right|_{\partial_{\mathrm{N}} \Omega}=0\right\}$. Then, for given input data $f_{1}(\cdot, t), f_{\mathrm{s}}(\cdot, t)$ in $H^{1}(\Omega)$, and initial data $\mathbf{X}(\cdot, 0)=\mathbf{X}_{0}, u_{\mathrm{s}}(\cdot, 0)=u_{\mathrm{s}, 0}, u_{1}(\cdot, 0)=u_{1,0}$, find time-dependent functions $\sigma_{\mathrm{l}}(\cdot, t)$ in $\mathbb{V}_{\mathrm{l}}(0), \boldsymbol{\sigma}_{\mathrm{s}}(\cdot, t)$ in $\mathbb{V}_{\mathrm{s}}, \mathbf{X}(\cdot, t)$ in $\mathbb{Y}, u_{1}(\cdot, t)$ in $\mathbb{Q}_{\mathrm{l}}, u_{\mathrm{s}}(\cdot, t)$ in $\mathbb{Q}_{\mathrm{s}}, \lambda(\cdot, t)$ in $\mathbb{M}$ such that

$$
\begin{aligned}
& \frac{1}{\widehat{K}_{1}} \int_{\Omega_{1}(t)} \sigma_{1} \cdot \eta_{1}-\int_{\Omega_{1}(t)} u_{1} \nabla \cdot \eta_{1}-\int_{\Gamma(t)} \lambda \eta_{1} \cdot \nu=-\int_{\partial \Omega} u_{\mathrm{D}} \eta_{1} \cdot \nu_{\Omega}, \quad \text { for all } \eta_{1} \in \mathbb{V}_{1}(0), \\
&-\int_{\Omega_{1}(t)} q_{1} \nabla \cdot \sigma_{1}-\int_{\Omega_{1}(t)} q_{1} \partial_{t} u_{1}=-\int_{\Omega_{1}(t)} q_{1} f_{\mathrm{l}}, \quad \text { for all } q_{1} \in \mathbb{Q}_{\mathrm{l}}, \\
& \frac{1}{\widehat{K}_{\mathrm{s}}} \int_{\Omega_{\mathrm{s}}(t)} \sigma_{\mathrm{s}} \cdot \boldsymbol{\eta}_{\mathrm{s}}-\int_{\Omega_{\mathrm{s}}(t)} u_{\mathrm{s}} \nabla \cdot \boldsymbol{\eta}_{\mathrm{s}}+\int_{\Gamma(t)} \lambda \boldsymbol{\eta}_{\mathrm{s}} \cdot \nu=0, \quad \text { for all } \boldsymbol{\eta}_{\mathrm{s}} \in \mathbb{V}_{\mathrm{s}}, \\
&-\int_{\Omega_{\mathrm{s}}(t)} q_{\mathrm{s}} \nabla \cdot \sigma_{\mathrm{s}}-\int_{\Omega_{\mathrm{s}}(t)} q_{\mathrm{s}} \partial_{t} u_{\mathrm{s}}=-\int_{\Omega_{\mathrm{s}}(t)} q_{\mathrm{s}} f_{\mathrm{s}}, \quad \text { for all } q_{\mathrm{s}} \in \mathbb{Q}_{\mathrm{s}}
\end{aligned}
$$




$$
\begin{aligned}
\int_{\Gamma(t)} \frac{1}{\widehat{\beta}(\boldsymbol{\nu})}\left(\partial_{t} \mathbf{X} \cdot \boldsymbol{\nu}\right)(\mathbf{Y} \cdot \boldsymbol{\nu})+\widehat{\alpha} \int_{\Gamma(t)} \nabla_{\Gamma} \mathbf{X}: \nabla_{\Gamma} \mathbf{Y}+\widehat{S} \int_{\Gamma(t)} \lambda(\mathbf{Y} \cdot \boldsymbol{\nu})=0, & \text { for all } \mathbf{Y} \in \mathbb{Y}, \\
\widehat{S} \int_{\Gamma(t)} \mu \partial_{t} \mathbf{X} \cdot \boldsymbol{\nu}-\int_{\Gamma(t)} \mu \boldsymbol{\sigma}_{1} \cdot \boldsymbol{\nu}+\int_{\Gamma(t)} \mu \boldsymbol{\sigma}_{\mathrm{s}} \cdot \boldsymbol{\nu}=0, & \text { for all } \mu \in \mathbb{M},
\end{aligned}
$$

where we have dropped the differential measure symbols $d \mathbf{x}, d S(\mathbf{x})$, etc., for brevity. Note: Integration by parts shows that $\lambda=u_{1}=u_{\mathrm{s}}$ on $\Gamma(t)$.

\subsection{Formal estimates}

Well-posedness of the fully continuous problem (3.11), (3.12) is challenging. One must handle the parameterized deforming domain appropriately and be able to obtain a priori estimates of the interface velocity, curvature, and improved regularity estimates of the variables [14, 31]. However, one may formally derive a priori bounds by assuming existence and uniqueness of a solution as well as sufficient regularity to allow for choosing test functions.

3.4.1 A priori bound. For simplicity, take $u_{\mathrm{D}}=0 . \operatorname{In}$ (3.11) and (3.12), choose $\eta_{\mathrm{l}}=\sigma_{\mathrm{l}}, \boldsymbol{\eta}_{\mathrm{s}}=\sigma_{\mathrm{s}}$, $\mathbf{Y}=\partial_{t} \mathbf{X}, q_{1}=-u_{1}, q_{\mathrm{s}}=-u_{\mathrm{s}}, \mu=-\lambda$, and add the equations together to get:

$$
\begin{aligned}
\frac{1}{\widehat{K}_{1}} \int_{\Omega_{1}(t)}\left|\sigma_{\mathrm{l}}\right|^{2}+ & \frac{1}{\widehat{K}_{\mathrm{s}}} \int_{\Omega_{\mathrm{s}}(t)}\left|\sigma_{\mathrm{s}}\right|^{2}+\int_{\Gamma(t)} \frac{1}{\widehat{\beta}(\nu)}\left|\partial_{t} \mathbf{X} \cdot \nu\right|^{2}+\widehat{\alpha} \int_{\Gamma(t)} \nabla_{\Gamma}\left(\partial_{t} \mathbf{X}\right): \nabla_{\Gamma} \mathbf{X} \\
& +\int_{\Omega_{1}(t)} u_{1} \partial_{t} u_{1}+\int_{\Omega_{\mathrm{s}}(t)} u_{\mathrm{s}} \partial_{t} u_{\mathrm{s}}=\int_{\Omega_{1}(t)} u_{1} f_{1}+\int_{\Omega_{\mathrm{s}}(t)} u_{\mathrm{s}} f_{\mathrm{s}} .
\end{aligned}
$$

Next, we make some preliminary calculations for some of the terms in (3.13). By standard shape differentiation $[16,32,56]$, we have

$$
\begin{aligned}
& \frac{d}{d t}\left(\int_{\Omega_{1}(t)} u_{1}^{2}\right)=\int_{\Omega_{1}(t)} \partial_{t}\left(u_{1}^{2}\right)-\int_{\Gamma(t)} u_{1}^{2}\left(\partial_{t} \mathbf{X}\right) \cdot \nu, \\
& \frac{d}{d t}\left(\int_{\Omega_{\mathrm{s}}(t)} u_{\mathrm{s}}^{2}\right)=\int_{\Omega_{\mathrm{s}}(t)} \partial_{t}\left(u_{\mathrm{s}}^{2}\right)+\int_{\Gamma(t)} u_{\mathrm{s}}^{2}\left(\partial_{t} \mathbf{X}\right) \cdot \boldsymbol{\nu},
\end{aligned}
$$

where we have accounted for the orientation of the normal vector $\nu$ of $\Gamma(t)$. Thus,

$$
\begin{aligned}
\int_{\Omega_{1}(t)} u_{1} \partial_{t} u_{1}+\int_{\Omega_{\mathrm{s}}(t)} u_{\mathrm{s}} \partial_{t} u_{\mathrm{s}} & =\frac{1}{2}\left(\int_{\Omega_{1}(t)} \partial_{t}\left(u_{1}^{2}\right)+\int_{\Omega_{\mathrm{s}}(t)} \partial_{t}\left(u_{\mathrm{s}}^{2}\right)\right) \\
& =\frac{1}{2} \frac{d}{d t}\left(\int_{\Omega_{1}(t)} u_{1}^{2}+\int_{\Omega_{\mathrm{s}}(t)} u_{\mathrm{s}}^{2}\right)+\frac{1}{2} \int_{\Gamma(t)}\left(u_{1}^{2}-u_{\mathrm{s}}^{2}\right) \partial_{t} \mathbf{X} \cdot \nu \\
& =\frac{1}{2} \frac{d}{d t}\left(\int_{\Omega_{1}(t)} u_{1}^{2}+\int_{\Omega_{\mathrm{s}}(t)} u_{\mathrm{s}}^{2}\right),
\end{aligned}
$$

where the last term is dropped because (formally) $u_{1}=u_{\mathrm{s}}$ on $\Gamma(t)$.

Now note that shape differentiation also tells us that $[16,32,56]$

$$
\int_{\Gamma(t)} \nabla_{\Gamma}\left(\partial_{t} \mathbf{X}\right): \nabla_{\Gamma} \mathbf{X}=\frac{d}{d t}|\Gamma(t)| .
$$


Therefore, we arrive at an identity

$$
\begin{gathered}
\int_{\Gamma(t)} \frac{1}{\widehat{\beta}(\boldsymbol{\nu})}\left[\left(\partial_{t} \mathbf{X}\right) \cdot \boldsymbol{\nu}\right]^{2}+\frac{1}{\widehat{K}_{1}}\left\|\sigma_{1}\right\|_{L^{2}\left(\Omega_{1}(t)\right)}^{2}+\frac{1}{\widehat{K}_{\mathrm{s}}}\left\|\sigma_{\mathrm{s}}\right\|_{L^{2}\left(\Omega_{\mathrm{s}}(t)\right)}^{2}+\widehat{\alpha} \frac{d}{d t}|\Gamma(t)| \\
+\frac{1}{2} \frac{d}{d t}\left(\int_{\Omega_{1}(t)} u_{1}^{2}+\int_{\Omega_{\mathrm{s}}(t)} u_{\mathrm{s}}^{2}\right)=\int_{\Omega_{1}(t)} u_{1} f_{1}+\int_{\Omega_{\mathrm{s}}(t)} u_{\mathrm{s}} f_{\mathrm{s}},
\end{gathered}
$$

which is a variation of a result in [5, eqn. (2.13)].

To continue, we recall a variant of a Grönwall type inequality in [43, Lemma 3.1].

LEMmA 3.2 Let $g, h, r, w:[0, T] \rightarrow \mathbb{R}$ be measurable and positive functions such that

$$
r^{2}(t)+g(t)+\int_{0}^{t} w(\tau) d \tau \leqslant r^{2}(0)+g(0)+\int_{0}^{t} r(\tau) h(\tau) d \tau, \quad \text { for all } t \in[0, T] .
$$

Then,

$$
r^{2}(t)+g(t)+\int_{0}^{t} w(\tau) d \tau \leqslant 2\left(r^{2}(0)+g(0)\right)+t \int_{0}^{t} h^{2}(\tau) d \tau, \quad \text { for all } t \in[0, T] .
$$

Now make the following identifications with the functions in Lemma 3.2:

$$
\begin{gathered}
h(t)=\sqrt{2}\left(\left\|f_{1}(t)\right\|_{L^{2}\left(\Omega_{1}(t)\right)}^{2}+\left\|f_{\mathrm{s}}(t)\right\|_{L^{2}\left(\Omega_{\mathrm{s}}(t)\right)}^{2}\right)^{1 / 2}, \\
r(t)=\frac{1}{\sqrt{2}}\left(\left\|u_{1}(t)\right\|_{L^{2}\left(\Omega_{1}(t)\right)}^{2}+\left\|u_{\mathrm{s}}(t)\right\|_{L^{2}\left(\Omega_{\mathrm{s}}(t)\right)}^{2}\right)^{1 / 2}, \quad g(t)=\widehat{\alpha}|\Gamma(t)|, \\
w(t)=\left\|\widehat{\beta}^{-1 / 2}(\boldsymbol{\nu})\left(\partial_{t} \mathbf{X}\right) \cdot \nu\right\|_{L^{2}(\Gamma(t))}^{2}+\frac{1}{\widehat{K}_{1}}\left\|\sigma_{1}(t)\right\|_{L^{2}\left(\Omega_{1}(t)\right)}^{2}+\frac{1}{\widehat{K}_{\mathrm{s}}}\left\|\sigma_{\mathrm{s}}(t)\right\|_{L^{2}\left(\Omega_{\mathrm{s}}(t)\right)}^{2} .
\end{gathered}
$$

Using Cauchy-Schwarz twice on the right-hand-side of (3.17) and integrating, we get

$$
\int_{0}^{t} w(\tau) d \tau+r^{2}(t)+g(t) \leqslant r^{2}(0)+g(0)+\int_{0}^{t} r(\tau) h(\tau) d \tau .
$$

Applying Lemma 3.2 delivers the a priori estimate:

$$
\begin{aligned}
& \frac{1}{2}\left(\left\|u_{1}(t)\right\|_{L^{2}\left(\Omega_{1}(t)\right)}^{2}+\left\|u_{\mathrm{s}}(t)\right\|_{L^{2}\left(\Omega_{\mathrm{s}}(t)\right)}^{2}\right)+\widehat{\alpha}|\Gamma(t)| \\
& +\int_{0}^{t}\left(\left\|\widehat{\beta}^{-1 / 2}(\boldsymbol{\nu})\left(\partial_{t} \mathbf{X}\right) \cdot \nu\right\|_{L^{2}(\Gamma(\tau))}^{2}+\frac{1}{\widehat{K}_{1}}\left\|\sigma_{1}(\tau)\right\|_{L^{2}\left(\Omega_{1}(\tau)\right)}^{2}+\frac{1}{\widehat{K}_{\mathrm{s}}}\left\|\sigma_{\mathrm{s}}(\tau)\right\|_{L^{2}\left(\Omega_{\mathrm{s}}(\tau)\right)}^{2}\right) d \tau \\
& \quad \leqslant\left(\left\|u_{1}(0)\right\|_{L^{2}\left(\Omega_{1}(0)\right)}^{2}+\left\|u_{\mathrm{s}}(0)\right\|_{L^{2}\left(\Omega_{\mathrm{s}}(0)\right)}^{2}\right)+2 \widehat{\alpha}|\Gamma(0)| \\
& \quad+2 t \int_{0}^{t}\left(\left\|f_{1}(\tau)\right\|_{L^{2}\left(\Omega_{1}(\tau)\right)}^{2}+\left\|f_{\mathrm{s}}(\tau)\right\|_{L^{2}\left(\Omega_{\mathrm{s}}(\tau)\right)}^{2}\right) d \tau, \quad \text { for all } t \in[0, T]
\end{aligned}
$$

See (4.27) for the semi-discrete version of (3.20). 
3.4.2 Conservation law. We also have a conservation law for the system which is simply a thermal energy balance. Choosing $q_{1}=1, q_{\mathrm{s}}=1$ in (3.11), and $\mu=1$ in (3.12) gives

$$
\begin{aligned}
-\int_{\partial_{\mathrm{D}} \Omega} \boldsymbol{\sigma}_{1} \cdot \nu_{\Omega} & +\int_{\Gamma(t)} \boldsymbol{\sigma}_{1} \cdot \boldsymbol{\nu}=\int_{\Omega_{\mathrm{l}}(t)} \partial_{t} u_{1}-\int_{\Omega_{\mathrm{l}}(t)} f_{\mathrm{l}} \\
-\int_{\Gamma(t)} \boldsymbol{\sigma}_{\mathrm{s}} \cdot \boldsymbol{\nu} & =\int_{\Omega_{\mathrm{s}}(t)} \partial_{t} u_{\mathrm{s}}-\int_{\Omega_{\mathrm{s}}(t)} f_{\mathrm{s}} \\
\widehat{S} \int_{\Gamma(t)}\left(\partial_{t} \mathbf{X}\right) \cdot \boldsymbol{\nu} & =\int_{\Gamma(t)} \sigma_{1} \cdot \boldsymbol{\nu}-\int_{\Gamma(t)} \boldsymbol{\sigma}_{\mathrm{s}} \cdot \boldsymbol{\nu}
\end{aligned}
$$

Adding them together gives the balance law:

$$
\int_{\Omega_{1}(t)} f_{1}+\int_{\Omega_{\mathrm{s}}(t)} f_{\mathrm{s}}-\int_{\partial_{\mathrm{D}} \Omega} \boldsymbol{\sigma}_{1} \cdot \nu_{\Omega}=\int_{\Omega_{1}(t)} \partial_{t} u_{1}+\int_{\Omega_{\mathrm{s}}(t)} \partial_{t} u_{\mathrm{s}}-\widehat{S} \int_{\Gamma(t)}\left(\partial_{t} \mathbf{X}\right) \cdot \boldsymbol{\nu},
$$

where the left side is the thermal (power) input and the right side is the rate of change in the stored thermal energy of the system. Note that energy is stored in the phase change associated with the velocity $\partial_{t} \mathbf{X}$ of $\Gamma(t)$. See (4.34) for the semi-discrete version of (3.21).

\section{Time semi-discrete formulation}

We now partition the time interval $(0, T)$ into subintervals of size $\Delta t$. We use a superscript $i$ to denote a time dependent quantity at time $t_{i}$. Furthermore, let $(\cdot, \cdot)_{\Sigma}$ denote the $L^{2}$ inner product on the generic domain $\Sigma$. For a general domain $\Sigma$, let $\langle\cdot, \cdot\rangle_{\Sigma}$ denote the duality pairing on $\Sigma$ between $H^{-1 / 2}(\Sigma)$ and $H^{1 / 2}(\Sigma)$ or between $H^{-1}(\Sigma)$ and $H^{1}(\Sigma)$ (the context will make it clear).

\subsection{Domain velocity}

4.1.1 Map $\Gamma^{i}$ to $\Gamma^{i+1}$. We introduce the interface velocity $\mathbf{V}:=\partial_{t} \mathbf{X}$ as a new variable. Thus, we approximate the interface position at time $t_{i+1}$ by a backward Euler scheme:

$$
\mathbf{X}^{i+1}=\mathbf{X}^{i}+\Delta t \mathbf{V}^{i+1}, \quad \text { where } \mathbf{V}^{i+1}: \Gamma^{i} \rightarrow \mathbb{R}^{3} \text {. }
$$

Thus, knowing $\mathbf{V}^{i+1}$ and $\mathbf{X}^{i}$ we can update the parametrization of the interface and obtain the interface $\Gamma^{i+1}$ at $t_{i+1}$. Note that $\mathbf{X}^{i}(\cdot) \equiv \operatorname{id}_{\Gamma^{i}}(\cdot)$ (the identity map) on $\Gamma^{i}$.

REMARK 4.1 We shall assume throughout this paper that $\mathbf{V}^{i+1}$ (for all $i$ ) is at least in $W^{1, \infty}\left(\Gamma^{i}\right.$ ) in order for the update (4.1) to make sense.

4.1.2 Map $\Omega_{1}^{i}$, $\Omega_{\mathrm{s}}^{i}$ to $\Omega_{1}^{i+1}, \Omega_{\mathrm{s}}^{i+1}$. Clearly, the bulk domains $\Omega_{\mathrm{l}}, \Omega_{\mathrm{s}}$ follow the interface $\Gamma$. Given $V^{i+1}$ on $\Gamma^{i}$, it can be extended to the entire domain $\Omega$ by a harmonic extension [22, 65], i.e. if $\mathbf{V}_{E}^{i+1}$ denotes the extension, then

$$
\mathbf{V}_{E}^{i+1} \in H^{1}(\Omega): \quad-\Delta \mathbf{V}_{E}^{i+1}=\mathbf{0}, \text { in } \Omega_{1}^{i} \cup \Omega_{\mathrm{s}}^{i}, \quad \mathbf{V}_{E}^{i+1}=\mathbf{V}^{i+1}, \text { on } \Gamma^{i}, \quad \mathbf{V}_{E}^{i+1}=\mathbf{0} \text {, on } \partial \Omega,
$$

In the following, we drop the $E$ subscript and use $\mathbf{V}^{i+1}$ to denote the extension. This induces a map $\Phi_{i+1}: \Omega^{i} \rightarrow \Omega^{i+1}$ for "updating" the domain:

$$
\Phi_{i+1}(\mathbf{x})=\operatorname{id}_{\Omega^{i}}(\mathbf{x})+\Delta t \mathbf{V}^{i+1}(\mathbf{x}), \quad \text { for all } \mathbf{x} \in \Omega^{i} .
$$


See $[27,28]$ for similar constructions in an ALE (Arbitrary-Lagrangian-Eulerian) context.

Note that $\Phi_{i+1}$ is defined over both $\Omega_{1}^{i}$ and $\Omega_{\mathrm{s}}^{i}$, and $\Omega_{1}^{i+1}:=\Phi_{i+1}\left(\Omega_{1}^{i}\right), \Omega_{\mathrm{s}}^{i+1}:=\Phi_{i+1}\left(\Omega_{\mathrm{s}}^{i}\right)$ conform to $\Gamma^{i+1}$. Similarly as for (4.1), we assume $\mathbf{V}^{i+1}$ (on $\Omega^{i}$ ) is at least in $W^{1, \infty}\left(\Omega^{i}\right)$. Furthermore, we assume $\Phi_{i+1}$ is a bijective map and $\operatorname{det}\left(\left[\nabla_{\mathbf{x}} \Phi_{i+1}(\mathbf{x})\right]\right)>0$. We note the following properties satisfied by $\Phi_{i+1}[33,58]$.

- If $\mathbf{y}=\Phi_{i+1}(\mathbf{x})$, then $\left(\nabla_{\mathbf{y}} \Phi_{i+1}^{-1} \circ \Phi_{i+1}\right)(\mathbf{x})=\left[\nabla_{\mathbf{x}} \Phi_{i+1}(\mathbf{x})\right]^{-1}$.

- If $f: \Omega^{i+1} \rightarrow \mathbb{R}$, then $\int_{\Omega^{i+1}} f(\mathbf{y}) d \mathbf{y}=\int_{\Omega^{i}} f\left(\Phi_{i+1}(\mathbf{x})\right) \operatorname{det}\left(\left[\nabla_{\mathbf{x}} \Phi_{i+1}(\mathbf{x})\right]\right) d \mathbf{x}$.

We use the map $\Phi_{i+1}$ to transform the functions $u_{1}^{i+1}, u_{\mathrm{s}}^{i+1}$ on $\Omega^{i}$ to new functions on $\Omega^{i+1}$ in order to advance the solution to the next time step (see (4.7)).

REMARK 4.2 (Time Step Restriction) In order for (4.3) to remain bijective, $\Delta t$ cannot be too large. In fact, it depends on $\left\|\nabla \mathbf{V}^{i+1}\right\|_{L^{\infty}(\Omega)}$ because $\operatorname{det}\left(\nabla \Phi_{i+1}\right)$ depends on $\nabla \mathbf{V}^{i+1}$. There is also a similar restriction on the time step in Theorem 4.14 (a priori bound).

4.1.3 Time derivative: Eulerian vs. Lagrangian. Similar to (4.1), we use a backward Euler method to discretize the temperature time derivatives at each time step:

$$
\left(\partial_{t} u_{1}\right)^{i+1} \approx \frac{u_{1}^{i+1}-u_{1}^{i}}{\Delta t}, \quad\left(\partial_{t} u_{\mathrm{s}}\right)^{i+1} \approx \frac{u_{\mathrm{s}}^{i+1}-u_{\mathrm{s}}^{i}}{\Delta t}
$$

But, because the domain is changing, $u_{j}^{i+1}, u_{j}^{i}(j=1, \mathrm{~s})$ are defined on different domains $\left(\Omega_{j}^{i}\right.$, $\Omega_{j}^{i-1}$, respectively; see next section). This means $u_{j}^{i}$ must be transferred to the new domain in order to compute the (discrete) Eulerian time derivative. The transference can be accomplished by an $L^{2}$ projection, for instance, but is not so convenient for a numerical method.

Therefore, we make use of the material derivative [58]. Using the standard formula $\dot{u}_{j}=\partial_{t} u_{j}+$ $\mathbf{V} \cdot \nabla u_{j}$, and introducing the flux variables, we have $\dot{u}_{j}=\partial_{t} u_{j}-\widehat{K}_{1}^{-1} \mathbf{V} \cdot \boldsymbol{\sigma}_{j}$ for $j=1$, s. Thus, we adopt the following discretization of $\partial_{t} u_{1}$ and $\partial_{t} u_{\mathrm{s}}$ :

$$
\left(\partial_{t} u_{j}\right)^{i+1} \approx \frac{u_{j}^{i+1}-u_{j}^{i} \circ \Phi_{i}^{-1}}{\Delta t}+\frac{1}{\widehat{K}_{j}}\left(\sigma_{j}^{i} \cdot \mathbf{V}^{i}\right) \circ \Phi_{i}^{-1}, \text { for } j=1, \mathrm{~s} .
$$

Note that we have treated the convective term explicitly, and (formally) taking $\Delta t \rightarrow 0$ recovers the standard material derivative formula. The advantage here is that computing $u_{j}^{i} \circ \Phi_{i}^{-1}$ and $\left(\sigma_{j}^{i} \cdot \mathbf{V}^{i}\right) \circ$ $\Phi_{i}^{-1}(j=1, \mathrm{~s})$, in the fully discrete method, is straightforward (see (5.3) and Remark 5.8).

\subsection{Weak formulation}

We now present the semi-discrete formulation of equations (3.11) and (3.12). The main idea is to write all integrals over the current domain $\Omega^{i}, \Gamma^{i}$ but set all of the solution variables at the next time step $t_{i+1}$ (i.e. a semi-implicit method). Moreover, we apply (4.1) and (4.4) and set $u_{\mathrm{D}}^{i+1}=$ $u_{\mathrm{D}}\left(\cdot, t_{i+1}\right), f_{\mathrm{l}}^{i+1}=f_{\mathrm{l}}\left(\cdot, t_{i+1}\right)$, and $f_{\mathrm{s}}^{i+1}=f_{\mathrm{s}}\left(\cdot, t_{i+1}\right)$. Thus, we arrive at the following weak formulation. At time $t_{i}$, find $\sigma_{1}^{i+1}$ in $\mathbb{V}_{1}^{i}(0), \sigma_{\mathrm{s}}^{i+1}$ in $\mathbb{V}_{\mathrm{s}}^{i}, \mathbf{V}^{i+1}$ in $\mathbb{Y}^{i}, u_{1}^{i+1}$ in $\mathbb{Q}_{1}^{i}, u_{\mathrm{s}}^{i+1}$ in $\mathbb{Q}_{\mathrm{s}}^{i}, \lambda^{i+1}$ 
in $\mathbb{M}^{i}$ such that

$$
\begin{aligned}
& \frac{1}{\widehat{K}_{1}}\left(\boldsymbol{\sigma}_{1}^{i+1}, \boldsymbol{\eta}_{1}\right)_{\Omega_{1}^{i}}-\left(u_{1}^{i+1}, \nabla \cdot \boldsymbol{\eta}_{1}\right)_{\Omega_{1}^{i}}-\left\langle\boldsymbol{\eta}_{1} \cdot \nu^{i}, \lambda^{i+1}\right\rangle_{\Gamma^{i}}=-\left\langle\boldsymbol{\eta}_{1} \cdot \nu_{\Omega}, u_{\mathrm{D}}^{i+1}\right\rangle_{\partial \Omega}, \\
& \text { for all } \eta_{1} \in \mathbb{V}_{1}^{i}(0) \text {, } \\
& -\left(\nabla \cdot \sigma_{1}^{i+1}, q_{1}\right)_{\Omega_{1}^{i}}-\frac{1}{\Delta t}\left(u_{1}^{i+1}, q_{1}\right)_{\Omega_{1}^{i}}+\frac{1}{\Delta t}\left(\bar{u}_{1}^{i}, q_{1}\right)_{\Omega_{1}^{i}}=-\left(f_{1}^{i+1}, q_{1}\right)_{\Omega_{1}^{i}} \text {, for all } q_{1} \in \mathbb{Q}_{1}^{i}, \\
& \frac{1}{\widehat{K}_{\mathrm{s}}}\left(\boldsymbol{\sigma}_{\mathrm{s}}^{i+1}, \boldsymbol{\eta}_{\mathrm{s}}\right)_{\Omega_{\mathrm{s}}^{i}}-\left(u_{\mathrm{s}}^{i+1}, \nabla \cdot \boldsymbol{\eta}_{\mathrm{s}}\right)_{\Omega_{\mathrm{s}}^{i}}+\left\langle\boldsymbol{\eta}_{\mathrm{s}} \cdot \boldsymbol{\nu}^{i}, \lambda^{i+1}\right\rangle_{\Gamma^{i}}=0 \text {, for all } \boldsymbol{\eta}_{\mathrm{s}} \in \mathbb{V}_{\mathrm{s}}^{i} \text {, } \\
& -\left(\nabla \cdot \sigma_{\mathrm{s}}^{i+1}, q_{\mathrm{s}}\right)_{\Omega_{\mathrm{s}}^{i}}-\frac{1}{\Delta t}\left(u_{\mathrm{s}}^{i+1}, q_{\mathrm{s}}\right)_{\Omega_{\mathrm{s}}^{i}}+\frac{1}{\Delta t}\left({\overline{u_{\mathrm{s}}}}^{i}, q_{\mathrm{s}}\right)_{\Omega_{\mathrm{s}}^{i}}=-\left(f_{\mathrm{s}}^{i+1}, q_{\mathrm{s}}\right)_{\Omega_{\mathrm{s}}^{i}} \text {, for all } q_{\mathrm{s}} \in \mathbb{Q}_{\mathrm{s}}^{i}, \\
& \left(\widehat{\beta}^{-1}\left(\boldsymbol{\nu}^{i}\right) \mathbf{V}^{i+1} \cdot \boldsymbol{\nu}^{i}, \mathbf{Y} \cdot \boldsymbol{\nu}^{i}\right)_{\Gamma^{i}}+\Delta t \widehat{\alpha}\left(\nabla_{\Gamma^{i}} \mathbf{V}^{i+1}, \nabla_{\Gamma^{i}} \mathbf{Y}\right)_{\Gamma^{i}} \\
& +\widehat{S}\left(\mathbf{Y} \cdot \nu^{i}, \lambda^{i+1}\right)_{\Gamma^{i}}=-\widehat{\alpha}\left(\nabla_{\Gamma^{i}} \mathbf{X}^{i}, \nabla_{\Gamma^{i}} \mathbf{Y}\right)_{\Gamma^{i}} \\
& \text { for all } \mathbf{Y} \in \mathbb{Y}^{i} \text {, }
\end{aligned}
$$

where the function spaces are defined over the current (known) domain $\Omega^{i}, \Gamma^{i}$. Then we use (4.1) to obtain the new interface position, which induces a map $\Phi_{i+1}: \Omega^{i} \rightarrow \Omega^{i+1}$. Because of (4.4), the temperature from the previous time index, $u_{j}^{i}: \Omega_{j}^{i-1} \rightarrow \mathbb{R}$, is mapped onto $\Omega_{j}^{i}$ by

$$
{\overline{u_{j}}}^{i}:=u_{j}^{i} \circ \Phi_{i}^{-1}-\Delta t \frac{1}{\widehat{K}_{j}}\left(\sigma_{j}^{i} \cdot \mathbf{V}^{i}\right) \circ \Phi_{i}^{-1}, \text { for } j=1, \mathrm{~s} .
$$

Iterating this procedure gives a time semi-discrete approximation of the fully continuous problem (3.11), (3.12).

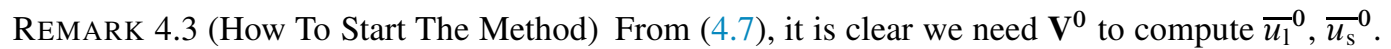
However, we start solving (4.5), (4.6) at $i=0$, which only gives $\mathbf{V}^{1}$. Hence, we must do one of the following. (i) specify $\mathbf{V}^{0}, \sigma_{1}^{0}, \sigma_{\mathrm{s}}^{0}$; (ii) set $\mathbf{V}^{0}=\sigma_{1}^{0}=\sigma_{\mathrm{s}}^{0}=\mathbf{0}$ (i.e. choose ${\overline{u_{1}}}^{0},{\overline{u_{\mathrm{s}}}}^{0}$ directly); (iii) or apply (ii) with a small time step to obtain an approximation of $\mathbf{V}^{0}, \sigma_{1}^{0}, \sigma_{\mathrm{s}}^{0}$. Either way, the error in approximating ${\overline{u_{1}}}^{0},{\overline{u_{\mathrm{s}}}}^{0}$ is only $O(\Delta t)$.

\subsection{Abstract formulation}

In order to simplify notation, we shall drop the time index notation and remember that we are solving for all variables on the current known domain $\Omega \equiv \Omega^{i}, \Gamma \equiv \Gamma^{i}$ with the current known normal vector $\boldsymbol{\nu} \equiv \boldsymbol{\nu}^{i}$. In particular, we take

$$
\begin{aligned}
& \sigma_{1}^{i+1} \equiv \sigma_{1}, \sigma_{\mathrm{s}}^{i+1} \equiv \sigma_{\mathrm{s}}, \quad \mathbf{V}^{i+1} \equiv \mathbf{V}, u_{1}^{i+1} \equiv u_{1}, u_{\mathrm{s}}^{i+1} \equiv u_{\mathrm{s}}, \lambda^{i+1} \equiv \lambda, \\
& f_{1}^{i+1} \equiv f_{1}, \quad f_{\mathrm{s}}^{i+1} \equiv f_{\mathrm{s}}, \quad u_{\mathrm{D}}^{i+1} \equiv u_{\mathrm{D}},{\overline{u_{\mathrm{l}}}}^{i} \equiv \overline{u_{1}},{\overline{u_{\mathrm{s}}}}^{i} \equiv \overline{u_{\mathrm{s}}}, \mathbf{X}^{i} \equiv \overline{\mathbf{X}}, \nabla_{\Gamma^{i}} \equiv \nabla_{\Gamma} .
\end{aligned}
$$


4.3.1 Bilinear and linear forms. For notational convenience, we introduce the following bilinear forms. The primal form is

$$
\begin{aligned}
a\left(\left(\boldsymbol{\eta}_{\mathrm{l}}, \boldsymbol{\eta}_{\mathrm{s}}, \mathbf{Y}\right),\left(\boldsymbol{\sigma}_{\mathrm{l}}, \boldsymbol{\sigma}_{\mathrm{s}}, \mathbf{V}\right)\right)= & \frac{1}{\widehat{K}_{1}}\left(\boldsymbol{\eta}_{\mathrm{l}}, \boldsymbol{\sigma}_{\mathrm{l}}\right)_{\Omega_{1}}+\frac{1}{\widehat{K}_{\mathrm{s}}}\left(\boldsymbol{\eta}_{\mathrm{s}}, \boldsymbol{\sigma}_{\mathrm{s}}\right)_{\Omega_{\mathrm{s}}} \\
& +\left(\widehat{\beta}^{-1}(\boldsymbol{\nu}) \mathbf{Y} \cdot \boldsymbol{\nu}, \mathbf{V} \cdot \boldsymbol{\nu}\right)_{\Gamma}+\Delta t \widehat{\alpha}\left(\nabla_{\Gamma} \mathbf{Y}, \nabla_{\Gamma} \mathbf{V}\right)_{\Gamma},
\end{aligned}
$$

the constraint form is

$$
\begin{aligned}
b\left(\left(\boldsymbol{\eta}_{\mathrm{l}}, \boldsymbol{\eta}_{\mathrm{s}}, \mathbf{Y}\right),\left(q_{\mathrm{l}}, q_{\mathrm{s}}, \mu\right)\right)= & -\left(\nabla \cdot \boldsymbol{\eta}_{\mathrm{l}}, q_{\mathrm{l}}\right)_{\Omega_{1}}-\left(\nabla \cdot \boldsymbol{\eta}_{\mathrm{s}}, q_{\mathrm{s}}\right)_{\Omega_{\mathrm{s}}} \\
& -\left\langle\boldsymbol{\eta}_{\mathbf{l}} \cdot \boldsymbol{\nu}, \mu\right\rangle_{\Gamma}+\left\langle\boldsymbol{\eta}_{\mathrm{s}} \cdot \boldsymbol{\nu}, \mu\right\rangle_{\Gamma}+\widehat{S}(\mathbf{Y} \cdot \boldsymbol{\nu}, \mu)_{\Gamma},
\end{aligned}
$$

and the lower diagonal form is

$$
c\left(\left(q_{1}, q_{\mathrm{s}}, \mu\right),\left(u_{1}, u_{\mathrm{s}}, \lambda\right)\right)=\frac{1}{\Delta t}\left(q_{1}, u_{1}\right)_{\Omega_{1}}+\frac{1}{\Delta t}\left(q_{\mathrm{s}}, u_{\mathrm{s}}\right)_{\Omega_{\mathrm{s}}} .
$$

The linear forms are defined by

$$
\begin{aligned}
& \chi\left(\boldsymbol{\eta}_{1}, \boldsymbol{\eta}_{\mathrm{s}}, \mathbf{Y}\right)=-\left(\left\langle\boldsymbol{\eta}_{1} \cdot \boldsymbol{\nu}_{\Omega}, u_{\mathrm{D}}\right\rangle_{\partial \Omega}+\widehat{\alpha}\left(\nabla_{\Gamma} \overline{\mathbf{X}}, \nabla_{\Gamma} \mathbf{Y}\right)_{\Gamma}\right) \\
& \psi\left(q_{1}, q_{\mathrm{s}}, \mu\right)=-\left(\left(f_{1}, q_{1}\right)_{\Omega_{1}}+\left(f_{\mathrm{s}}, q_{\mathrm{s}}\right)_{\Omega_{\mathrm{s}}}+\frac{1}{\Delta t}\left(\overline{u_{1}}, q_{1}\right)_{\Omega_{1}}+\frac{1}{\Delta t}\left(\overline{u_{\mathrm{s}}}, q_{\mathrm{s}}\right)_{\Omega_{\mathrm{s}}}\right) .
\end{aligned}
$$

4.3.2 Saddle-point formulation. Define the primal space by

$$
\mathbb{Z}=\mathbb{V}_{1}(0) \times \mathbb{V}_{\mathrm{s}} \times \mathbb{Y}
$$

and the multiplier space by

$$
\mathbb{T}=\mathbb{Q}_{1} \times \mathbb{Q}_{\mathrm{s}} \times \mathbb{M} \text {. }
$$

With the above notation, the formulation (4.5), (4.6) can be written as a saddle-point problem.

VARIATIONAL Formulation 4.4 Find $\left(\sigma_{1}, \sigma_{\mathrm{s}}, \mathbf{V}\right)$ in $\mathbb{V}_{1}(0) \times \mathbb{V}_{\mathrm{s}} \times \mathbb{Y}$ and $\left(u_{1}, u_{\mathrm{s}}, \lambda\right)$ in $\mathbb{Q}_{1} \times \mathbb{Q}_{\mathrm{s}} \times \mathbb{M}$ such that

$$
\begin{gathered}
a\left(\left(\boldsymbol{\eta}_{1}, \boldsymbol{\eta}_{\mathrm{s}}, \mathbf{Y}\right),\left(\boldsymbol{\sigma}_{1}, \boldsymbol{\sigma}_{\mathrm{s}}, \mathbf{V}\right)\right)+b\left(\left(\boldsymbol{\eta}_{1}, \boldsymbol{\eta}_{\mathrm{s}}, \mathbf{Y}\right),\left(u_{1}, u_{\mathrm{s}}, \lambda\right)\right)=\chi\left(\boldsymbol{\eta}_{1}, \boldsymbol{\eta}_{\mathrm{s}}, \mathbf{Y}\right), \\
b\left(\left(\boldsymbol{\sigma}_{1}, \boldsymbol{\sigma}_{\mathrm{s}}, \mathbf{V}\right),\left(q_{1}, q_{\mathrm{s}}, \mu\right)\right)-c\left(\left(q_{1}, q_{\mathrm{s}}, \mu\right),\left(u_{1}, u_{\mathrm{s}}, \lambda\right)\right)=\psi\left(q_{1}, q_{\mathrm{s}}, \mu\right),
\end{gathered}
$$

for all $\left(\boldsymbol{\eta}_{1}, \boldsymbol{\eta}_{\mathrm{s}}, \mathbf{Y}\right)$ in $\mathbb{V}_{1}(0) \times \mathbb{V}_{\mathrm{s}} \times \mathbb{Y}$, and $\left(q_{1}, q_{\mathrm{s}}, \mu\right)$ in $\mathbb{Q}_{1} \times \mathbb{Q}_{\mathrm{s}} \times \mathbb{M}$. The temperatures $u_{1}, u_{\mathrm{s}}$ are Lagrange multipliers as well as the interface temperature $\lambda$.

\section{$4.4 \quad$ Norms}

4.4.1 Non-degenerate interface. The purpose of the following assumption is to avoid a case where $\Gamma$ is closed and very flat (e.g. the surface of a pancake). It is necessary to ensure the equivalence of the norms in Proposition 4.6.

ASSUMPTION 4.5 Assume that $\Gamma$ is a Lipschitz or polyhedral manifold. In addition, for any nonzero constant vector $\mathbf{a} \in \mathbb{R}^{3}$, assume there exists an open neighborhood $n \subset \Gamma$ such that $|\eta| \geqslant$ $c_{0}>0$ and

$$
\mathbf{a} \cdot \boldsymbol{\nu}(\mathbf{x})>0, \quad \forall \mathbf{x} \in \eta, \quad \text { or } \quad \mathbf{a} \cdot \boldsymbol{\nu}(\mathbf{x})<0, \quad \forall \mathbf{x} \in \eta
$$


4.4.2 Primal norm. Clearly, $\left\|\left(\boldsymbol{\eta}_{\mathrm{l}}, \boldsymbol{\eta}_{\mathrm{s}}, \mathbf{Y}\right)\right\|_{\mathbb{Z}^{\circ}}^{2}:=\left\|\boldsymbol{\eta}_{1}\right\|_{H\left(\mathrm{div}, \Omega_{1}\right)}^{2}+\left\|\boldsymbol{\eta}_{\mathrm{s}}\right\|_{H\left(\mathrm{div}, \Omega_{\mathrm{s}}\right)}^{2}+\|\mathbf{Y}\|_{H^{1}(\Gamma)}^{2}$ is a norm on $\mathbb{Z}$. But because of the form of the equations, we shall use a different norm. First, we note an equivalent norm to the standard $H^{1}$ norm on $\Gamma$ (recall that $\|\mathbf{Y}\|_{H^{1}(\Gamma)}^{2}=\|\mathbf{Y}\|_{L^{2}(\Gamma)}^{2}+\left\|\nabla_{\Gamma} \mathbf{Y}\right\|_{L^{2}(\Gamma)}^{2}$ ). PROPOSITION 4.6 Let $\Gamma$ be a Lipschitz or polyhedral manifold. Define:

$$
\||\mathbf{Y}|\|^{2}=\|\mathbf{Y} \cdot \boldsymbol{\nu}\|_{H^{-1 / 2}(\Gamma)}^{2}+\left\|\nabla_{\Gamma} \mathbf{Y}\right\|_{L^{2}(\Gamma)}^{2} .
$$

Then, ||$|\mathbf{Y}||| \approx\|\mathbf{Y}\|_{H^{1}(\Gamma)}$, with constants that only depend on the domain.

Proof. First, verify that $\||\mathbf{Y}|||$ is a norm on $H^{1}(\Gamma)$. We just need to check that $\||| \mathbf{Y} \mid\|=0 \Leftrightarrow \mathbf{Y}=\mathbf{0}$ since the other norm properties are trivial to verify. If $\|\mid \mathbf{Y}\|=0$, then $\left\|\nabla_{\Gamma} \mathbf{Y}\right\|_{L^{2}(\Gamma)}=0$, so $\mathbf{Y}=\mathbf{a} \in \mathbb{R}^{3}$ (constant vector). If $\mathbf{a} \neq \mathbf{0}$, then by Assumption 4.5, $\mathbf{a} \cdot \boldsymbol{\nu}>0$ (or $<0$ ) on a set of positive measure. Thus, $\|\mathbf{Y} \cdot \boldsymbol{\nu}\|_{H^{-1 / 2}(\Gamma)}^{2} \neq 0$, but this is a contradiction, so then $\mathbf{a}=\mathbf{0}$. Since |I| $\mid \|$ is a norm on $H^{1}(\Gamma)$, the equivalence with $\|\cdot\|_{H^{1}(\Gamma)}$ follows by a classical compactness argument $[1,21]$.

In light of the above, we define the following primal norm:

$$
\begin{aligned}
\left\|\left(\boldsymbol{\eta}_{\mathrm{l}}, \boldsymbol{\eta}_{\mathrm{s}}, \mathbf{Y}\right)\right\|_{\mathbb{Z}}^{2}=\frac{1}{\widehat{K}_{1}}\left\|\boldsymbol{\eta}_{1}\right\|_{H\left(\operatorname{div}, \Omega_{1}\right)}^{2}+\frac{1}{\widehat{K}_{\mathrm{s}}}\left\|\boldsymbol{\eta}_{\mathrm{s}}\right\|_{H\left(\mathrm{div}, \Omega_{\mathrm{s}}\right)}^{2}+\left\|\widehat{\beta}^{-1 / 2} \mathbf{Y} \cdot \boldsymbol{\nu}\right\|_{L^{2}(\Gamma)}^{2} \\
+\|\mathbf{Y} \cdot \boldsymbol{\nu}\|_{H^{-1 / 2}(\Gamma)}^{2}+\Delta t \widehat{\alpha}\left\|\nabla_{\Gamma} \mathbf{Y}\right\|_{L^{2}(\Gamma)}^{2} .
\end{aligned}
$$

The choice of $H^{-1 / 2}(\Gamma)$ is the most convenient for our formulation.

4.4.3 Multiplier norm. The obvious multiplier norm is $\left\|\left(q_{1}, q_{\mathrm{s}}, \mu\right)\right\|_{\mathbb{T}^{\diamond}}^{2}:=\left\|q_{1}\right\|_{L^{2}\left(\Omega_{1}\right)}^{2}+$ $\left\|q_{\mathrm{s}}\right\|_{L^{2}\left(\Omega_{\mathrm{s}}\right)}^{2}+\|\mu\|_{H^{1 / 2}(\Gamma)}^{2}$. However, because of the form of the bilinear form $b$ (4.9), it is more advantageous to use the following equivalent norm:

$$
\begin{aligned}
\left\|\left(q_{1}, q_{\mathrm{s}}, \mu\right)\right\|_{\mathbb{T}}^{2}=\left\|\tilde{q}_{1}\right\|_{L^{2}\left(\Omega_{1}\right)}^{2}+\left\|\tilde{q}_{\mathrm{s}}\right\|_{L^{2}\left(\Omega_{\mathrm{s}}\right)}^{2}+\| \mu & -\hat{q}_{1} \|_{H_{1}^{1 / 2}(\Gamma)}^{2} \\
& +\left\|\mu-\hat{q}_{\mathrm{s}}\right\|_{H_{\mathrm{s}}^{1 / 2}(\Gamma)}^{2}+\widehat{S}\|\mu \nu\|_{H^{-1}(\Gamma)}^{2}
\end{aligned}
$$

where we introduced the mean value: $\hat{q}_{i}:=\frac{1}{\left|\Omega_{i}\right|} \int_{\Omega_{i}} q_{i}$, and $\tilde{q_{i}}:=q_{i}-\hat{q}_{i}$ (for $i=1$, s). We also define the mean value on $\Gamma: \hat{\mu}:=\frac{1}{|\Gamma|} \int_{\Gamma} \mu$, and $\tilde{\mu}:=\mu-\hat{\mu}$.

Proposition 4.7 (Equivalence of Multiplier Norms) Let $\Gamma$ be a Lipschitz or polyhedral manifold. Then, $\left\|\left(q_{1}, q_{\mathrm{s}}, \mu\right)\right\|_{\mathbb{T}^{\diamond}} \approx\left\|\left(q_{1}, q_{\mathrm{s}}, \mu\right)\right\|_{\mathbb{T}}$, with constants that only depend on the domain and $\widehat{S}$.

Proof. Again, use a compactness argument.

\subsection{Well-posedness}

This section verifies the conditions needed for well-posedness of (4.14) $[9,11]$. 


\subsubsection{Main conditions.}

LEMMA 4.8 (Continuity of Forms) For all $\left(\boldsymbol{\eta}_{\mathbf{l}}, \boldsymbol{\eta}_{\mathrm{s}}, \mathbf{Y}\right),\left(\boldsymbol{\sigma}_{1}, \boldsymbol{\sigma}_{\mathrm{s}}, \mathbf{V}\right)$ in $\mathbb{Z}$ and $\left(q_{1}, q_{\mathrm{s}}, \mu\right),\left(u_{1}, u_{\mathrm{s}}, \lambda\right)$ in $\mathbb{T}$,

$$
\begin{aligned}
\left|a\left(\left(\boldsymbol{\eta}_{1}, \boldsymbol{\eta}_{\mathrm{s}}, \mathbf{Y}\right),\left(\boldsymbol{\sigma}_{1}, \boldsymbol{\sigma}_{\mathrm{s}}, \mathbf{V}\right)\right)\right| & \leqslant C_{a}\left\|\left(\boldsymbol{\eta}_{\mathrm{l}}, \boldsymbol{\eta}_{\mathrm{s}}, \mathbf{Y}\right)\right\|_{\mathbb{Z}}\left\|\left(\boldsymbol{\sigma}_{\mathrm{l}}, \boldsymbol{\sigma}_{\mathrm{s}}, \mathbf{V}\right)\right\|_{\mathbb{Z}}, \\
\left|b\left(\left(\boldsymbol{\eta}_{\mathrm{l}}, \boldsymbol{\eta}_{\mathrm{s}}, \mathbf{Y}\right),\left(q_{1}, q_{\mathrm{s}}, \mu\right)\right)\right| & \leqslant C_{b}\left\|\left(\boldsymbol{\eta}_{1}, \boldsymbol{\eta}_{\mathrm{s}}, \mathbf{Y}\right)\right\|_{\mathbb{Z}}\left\|\left(q_{1}, q_{\mathrm{s}}, \mu\right)\right\|_{\mathbb{T}}, \\
\left|c\left(\left(q_{1}, q_{\mathrm{s}}, \mu\right),\left(u_{1}, u_{\mathrm{s}}, \lambda\right)\right)\right| & \leqslant \Delta t^{-1}\left(\left\|q_{1}\right\|_{L^{2}\left(\Omega_{1}\right)}\left\|u_{1}\right\|_{L^{2}\left(\Omega_{1}\right)}+\left\|q_{\mathrm{s}}\right\|_{L^{2}\left(\Omega_{\mathrm{s}}\right)}\left\|u_{\mathrm{s}}\right\|_{L^{2}\left(\Omega_{\mathrm{s}}\right)}\right), \\
\left|\chi\left(\boldsymbol{\eta}_{1}, \boldsymbol{\eta}_{\mathrm{s}}, \mathbf{Y}\right)\right| & \leqslant C_{\chi}\left\|\left(\boldsymbol{\eta}_{1}, \boldsymbol{\eta}_{\mathrm{s}}, \mathbf{Y}\right)\right\|_{\mathbb{Z}}, \\
\left|\psi\left(q_{1}, q_{\mathrm{s}}, \mu\right)\right| & \leqslant C_{\psi}\left\|\left(q_{1}, q_{\mathrm{s}}, \mu\right)\right\|_{\mathbb{T}},
\end{aligned}
$$

where $C_{a}, C_{b}, C_{\chi}, C_{\psi}>0$ are constants that depend on physical parameters and domain geometry. In addition, $C_{\chi}$ depends on $u_{\mathrm{D}}, \Delta t^{-1 / 2}$, and $C_{\psi}$ depends on $f_{1}, f_{\mathrm{s}}, \overline{u_{1}}, \overline{u_{\mathrm{s}}}$ and $\Delta t^{-1}$.

Proof. The first result comes from two uses of the Schwarz inequality. The second estimate follows by noting

$$
\begin{aligned}
& -\left(\nabla \cdot \boldsymbol{\eta}_{1}, q_{1}\right)_{\Omega_{1}}-\left\langle\boldsymbol{\eta}_{1} \cdot \boldsymbol{\nu}, \mu\right\rangle_{\Gamma} \leqslant\left(\left\|q_{1}\right\|_{L^{2}\left(\Omega_{1}\right)}+\|\mu\|_{H_{1}^{1 / 2}(\Gamma)}\right)\left\|\boldsymbol{\eta}_{1}\right\|_{H\left(\operatorname{div}, \Omega_{1}\right)}, \\
& -\left(\nabla \cdot \boldsymbol{\eta}_{\mathrm{s}}, q_{\mathrm{s}}\right)_{\Omega_{\mathrm{s}}}+\left\langle\boldsymbol{\eta}_{\mathrm{s}} \cdot \boldsymbol{\nu}, \mu\right\rangle_{\Gamma} \leqslant\left(\left\|q_{\mathrm{s}}\right\|_{L^{2}\left(\Omega_{\mathrm{s}}\right)}+\|\mu\|_{H_{\mathrm{s}}^{1 / 2}(\Gamma)}\right)\left\|\boldsymbol{\eta}_{\mathrm{s}}\right\|_{H\left(\operatorname{div}, \Omega_{\mathrm{s}}\right)},
\end{aligned}
$$

where we used Cauchy-Schwarz and (3.7). In addition, by (3.8), we have

$$
\widehat{S} \int_{\Gamma} \mu(\mathbf{Y} \cdot \boldsymbol{\nu})=\widehat{S}\langle\mathbf{Y} \cdot \boldsymbol{\nu}, \mu\rangle_{\Gamma} \leqslant \widehat{S}\|\mathbf{Y} \cdot \boldsymbol{\nu}\|_{H^{-1 / 2}(\Gamma)}\|\mu\|_{H^{1 / 2}(\Gamma)}
$$

The bound on $b$ then follows by combining these results and using Proposition 4.7. The bound on $c$ is obvious. Next, we have

$$
\chi\left(\boldsymbol{\eta}_{1}, \boldsymbol{\eta}_{\mathrm{s}}, \mathbf{Y}\right) \leqslant\left\|u_{\mathrm{D}}\right\|_{H^{1 / 2}(\partial \Omega)}\left\|\boldsymbol{\eta}_{1} \cdot \nu_{\Omega}\right\|_{H^{-1 / 2}(\partial \Omega)}+C_{1} \widehat{\alpha}\left\|\nabla_{\Gamma} \mathbf{Y}\right\|_{L^{2}(\Gamma)} \leqslant C\left\|\left(\boldsymbol{\eta}_{1}, \boldsymbol{\eta}_{\mathrm{s}}, \mathbf{Y}\right)\right\|_{\mathbb{Z}},
$$

where $C$ depends on $\Delta t^{-1 / 2}$ and the data $u_{\mathrm{D}}$. The last inequality follows from (4.11) where the constant depends on $\Delta t^{-1}$ and the problem data.

LEMMA 4.9 (Coercivity) Let $\left(\boldsymbol{\eta}_{\mathrm{l}}, \boldsymbol{\eta}_{\mathrm{s}}, \mathbf{Y}\right) \in \mathbb{Z}$ with $b\left(\left(\boldsymbol{\eta}_{1}, \boldsymbol{\eta}_{\mathrm{s}}, \mathbf{Y}\right),\left(q_{1}, q_{\mathrm{s}}, \mu\right)\right)=0$ for all $\left(q_{1}, q_{\mathrm{s}}, \mu\right) \in$ $\mathbb{T}$. Then,

$$
\left|a\left(\left(\boldsymbol{\eta}_{1}, \boldsymbol{\eta}_{\mathrm{s}}, \mathbf{Y}\right),\left(\boldsymbol{\eta}_{\mathrm{l}}, \boldsymbol{\eta}_{\mathrm{s}}, \mathbf{Y}\right)\right)\right| \geqslant C\left\|\left(\boldsymbol{\eta}_{\mathrm{l}}, \boldsymbol{\eta}_{\mathrm{s}}, \mathbf{Y}\right)\right\|_{\mathbb{Z}}^{2},
$$

where $C>0$ is a constant depending on $\widehat{S}, \widehat{K}_{1}, \widehat{K_{\mathrm{s}}}$, and the domain. This is true even if $\widehat{\beta} \rightarrow \infty$.

Proof. From (4.8), we get

$$
\begin{aligned}
a\left(\left(\boldsymbol{\eta}_{1}, \boldsymbol{\eta}_{\mathrm{s}}, \mathbf{Y}\right),\left(\boldsymbol{\eta}_{\mathrm{l}}, \boldsymbol{\eta}_{\mathrm{s}}, \mathbf{Y}\right)\right) \geqslant & \frac{1}{\widehat{K}_{1}}\left\|\boldsymbol{\eta}_{\mathbf{l}}\right\|_{L^{2}\left(\Omega_{1}\right)}^{2}+\frac{1}{\widehat{K}_{\mathrm{s}}}\left\|\boldsymbol{\eta}_{\mathrm{s}}\right\|_{L^{2}\left(\Omega_{\mathrm{s}}\right)}^{2}+\left\|\widehat{\beta}^{-1 / 2} \mathbf{Y} \cdot \boldsymbol{\nu}\right\|_{L^{2}(\Gamma)}^{2} \\
& +\Delta t \widehat{\alpha}\left\|\nabla_{\Gamma} \mathbf{Y}\right\|_{L^{2}(\Gamma)}^{2} \\
= & \frac{1}{\widehat{K}_{1}}\left\|\boldsymbol{\eta}_{1}\right\|_{H\left(\operatorname{div}, \Omega_{1}\right)}^{2}+\frac{1}{\widehat{K}_{\mathrm{s}}}\left\|\boldsymbol{\eta}_{\mathrm{s}}\right\|_{H\left(\operatorname{div}, \Omega_{\mathrm{s}}\right)}^{2} \\
& +\left\|\widehat{\beta}^{-1 / 2} \mathbf{Y} \cdot \boldsymbol{\nu}\right\|_{L^{2}(\Gamma)}^{2}+\Delta t \widehat{\alpha}\left\|\nabla_{\Gamma} \mathbf{Y}\right\|_{L^{2}(\Gamma)}^{2}
\end{aligned}
$$


where the last step follows from the hypothesis $\nabla \cdot \boldsymbol{\eta}_{1}=\nabla \cdot \boldsymbol{\eta}_{\mathrm{s}}=0$. Also by hypothesis, we have

$$
\widehat{S}(\mathbf{Y} \cdot \boldsymbol{\nu}, \mu)_{\Gamma}=\left\langle\boldsymbol{\eta}_{1} \cdot \boldsymbol{\nu}, \mu\right\rangle_{\Gamma}-\left\langle\boldsymbol{\eta}_{\mathrm{s}} \cdot \boldsymbol{\nu}, \mu\right\rangle_{\Gamma}, \quad \text { for all } \mu \in H^{1 / 2}(\Gamma) .
$$

Hence, using (3.7) and (3.8), we have

$$
\widehat{S}\|\mathbf{Y} \cdot \boldsymbol{\nu}\|_{H^{-1 / 2}(\Gamma)}=\sup _{\mu \in H^{1 / 2}(\Gamma)} \frac{\widehat{S}(\mathbf{Y} \cdot \boldsymbol{\nu}, \mu)_{\Gamma}}{\|\mu\|_{H^{1 / 2}(\Gamma)}} \leqslant 2\left(\left\|\boldsymbol{\eta}_{1}\right\|_{H\left(\mathrm{div}, \Omega_{1}\right)}+\left\|\boldsymbol{\eta}_{\mathrm{s}}\right\|_{H\left(\mathrm{div}, \Omega_{\mathrm{s}}\right)}\right) .
$$

Combining these inequalities yields the assertion.

LEMma 4.10 (Inf-Sup) For all $\left(q_{1}, q_{\mathrm{s}}, \mu\right) \in \mathbb{T}$, the following "inf-sup" condition holds

$$
\sup _{\left(\boldsymbol{\eta}_{\mathrm{l}}, \boldsymbol{\eta}_{\mathrm{s}}, \mathbf{Y}\right) \in \mathbb{Z}} \frac{b\left(\left(\boldsymbol{\eta}_{1}, \boldsymbol{\eta}_{\mathrm{s}}, \mathbf{Y}\right),\left(q_{1}, q_{\mathrm{s}}, \mu\right)\right)}{\left\|\left(\boldsymbol{\eta}_{1}, \boldsymbol{\eta}_{\mathrm{s}}, \mathbf{Y}\right)\right\|_{\mathbb{Z}^{\circ}}} \geqslant C\left\|\left(q_{1}, q_{\mathrm{s}}, \mu\right)\right\|_{\mathbb{T}},
$$

where $C>0$ depends on the domain and $\widehat{S}$. If $\left\|\left(\boldsymbol{\eta}_{1}, \boldsymbol{\eta}_{\mathrm{s}}, \mathbf{Y}\right)\right\|_{\mathbb{Z}^{\circ}}$ is replaced by $\left\|\left(\boldsymbol{\eta}_{1}, \boldsymbol{\eta}_{\mathrm{s}}, \mathbf{Y}\right)\right\|_{\mathbb{Z}}$ in the denominator, then the inf-sup still holds, except $C$ also depends on $\widehat{K}_{1}, \widehat{K}_{\mathrm{s}}, \widehat{\alpha}$, and $\widehat{\beta}_{-}$. Furthermore, $C$ does not depend on the time step $\Delta t$, as long as $\Delta t \leqslant 1$.

Proof. Setting $\eta_{1} \cdot \nu_{\Omega}=0$ on $\partial \Omega$, and accounting for the orientation of the normal vector and using the divergence theorem, we have

$$
\begin{aligned}
& b\left(\left(\boldsymbol{\eta}_{\mathrm{l}}, \boldsymbol{\eta}_{\mathrm{s}}, \mathbf{Y}\right),\left(q_{\mathrm{l}}, q_{\mathrm{s}}, \mu\right)\right) \\
&=-\left(\nabla \cdot \boldsymbol{\eta}_{\mathrm{l}}, q_{1}\right)_{\Omega_{1}}-\left(\nabla \cdot \boldsymbol{\eta}_{\mathrm{s}}, q_{\mathrm{s}}\right)_{\Omega_{\mathrm{s}}}-\left\langle\boldsymbol{\eta}_{1} \cdot \boldsymbol{\nu}, \mu\right\rangle_{\Gamma}+\left\langle\boldsymbol{\eta}_{\mathrm{s}} \cdot \boldsymbol{\nu}, \mu\right\rangle_{\Gamma}+\widehat{S}(\mathbf{Y} \cdot \boldsymbol{\nu}, \mu)_{\Gamma} \\
&=-\left(\nabla \cdot \boldsymbol{\eta}_{1}, \tilde{q}_{1}\right)_{\Omega_{1}}-\left(\nabla \cdot \boldsymbol{\eta}_{\mathrm{s}}, \tilde{q}_{\mathrm{s}}\right)_{\Omega_{\mathrm{s}}}-\left\langle\boldsymbol{\eta}_{1} \cdot \boldsymbol{\nu}, \mu-\hat{q}_{1}\right\rangle_{\Gamma}+\left\langle\boldsymbol{\eta}_{\mathrm{s}} \cdot \boldsymbol{\nu}, \mu-\hat{q}_{\mathrm{s}}\right\rangle_{\Gamma} \\
&+\widehat{S}(\mathbf{Y} \cdot \boldsymbol{\nu}, \mu)_{\Gamma} .
\end{aligned}
$$

By definition of the $H_{1}^{1 / 2}(\Gamma)$ norm (3.7), there exists a $\xi \in \mathbb{V}_{1}(0)$ such that $-\left\langle\xi \cdot \nu, \mu-\hat{q}_{1}\right\rangle_{\Gamma}=$ $\left\|\mu-\hat{q}_{1}\right\|_{H_{1}^{1 / 2}(\Gamma)}$ and $\|\boldsymbol{\xi}\|_{H\left(\mathrm{div}, \Omega_{1}\right)}=1$. With this, we construct the vector field $\boldsymbol{\eta}_{1} \in H\left(\operatorname{div}, \Omega_{1}\right)$. Let $\phi_{1}, \phi_{2}$ in $H^{1}\left(\Omega_{1}\right)$ (with zero mean value) be weak solutions of the following elliptic problems,

$$
\begin{aligned}
& -\Delta \phi_{1}=\frac{\tilde{q}_{1}}{\left\|\tilde{q}_{1}\right\|_{L^{2}\left(\Omega_{1}\right)}}, \text { in } \Omega_{1}, \quad \boldsymbol{\nu} \cdot \nabla \phi_{1}=0, \text { on } \partial \Omega_{1} \equiv \Gamma \cup \partial \Omega, \\
& -\Delta \phi_{2}=\frac{1}{\left|\Omega_{1}\right|} \int_{\partial \Omega_{1}}^{\xi} \boldsymbol{\boldsymbol { \nu }}, \text { in } \Omega_{1}, \quad \boldsymbol{\nu} \cdot \nabla \phi_{2}=\boldsymbol{\xi} \cdot \boldsymbol{\nu}, \text { on } \partial \Omega_{1},
\end{aligned}
$$

and define $\eta_{1}=\nabla \phi_{1}+\nabla \phi_{2}$ (note that $\nabla \phi_{1}$ and $\nabla \phi_{2}$ are in $\mathbb{V}_{1}(0)$ ). This gives

$$
\begin{aligned}
-\left(\nabla \cdot \boldsymbol{\eta}_{1}, \tilde{q}_{1}\right)_{\Omega_{1}}-\left\langle\boldsymbol{\eta}_{1} \cdot \boldsymbol{\nu}, \mu-\hat{q}_{1}\right\rangle_{\Gamma} & =\left(-\Delta \phi_{1}, \tilde{q}_{1}\right)_{\Omega_{1}}+\left(-\Delta \phi_{2}, \tilde{q}_{1}\right)_{\Omega_{1}}-\left\langle\boldsymbol{\nu} \cdot \nabla \phi_{2}, \mu-\hat{q}_{1}\right\rangle_{\Gamma} \\
& =\left\|\tilde{q}_{1}\right\|_{L^{2}\left(\Omega_{1}\right)}+\left\|\mu-\hat{q}_{1}\right\|_{H_{1}^{1 / 2}(\Gamma)} .
\end{aligned}
$$

Now bound $\left\|\boldsymbol{\eta}_{1}\right\|_{H\left(\mathrm{div}, \Omega_{1}\right)}$. Since (3.2) and (3.3) hold with $\Omega$ replaced by $\Omega_{1}$, we get $\left\|\Delta \phi_{2}\right\|_{L^{2}\left(\Omega_{1}\right)} \leqslant$ $C_{0}\left|\langle\boldsymbol{\xi} \cdot \boldsymbol{\nu}, 1\rangle_{\partial \Omega_{1}}\right| \leqslant C_{1}\|\boldsymbol{\xi} \cdot \boldsymbol{\nu}\|_{H^{-1 / 2}\left(\partial \Omega_{1}\right)} \leqslant C_{1}\|\boldsymbol{\xi}\|_{H\left(\mathrm{div}, \Omega_{1}\right)}=C_{1}$. Moreover, a standard a priori bound gives $\left\|\phi_{2}\right\|_{H^{1}\left(\Omega_{1}\right)} \leqslant C_{2}\left(\left\|\Delta \phi_{2}\right\|_{L^{2}\left(\Omega_{1}\right)}+\|\boldsymbol{\xi} \cdot \nu\|_{H^{-1 / 2}\left(\partial \Omega_{1}\right)}\right) \leqslant C_{2}\left(C_{1}+1\right)$. Similarly, we deduce that $\left\|\Delta \phi_{1}\right\|_{L^{2}\left(\Omega_{1}\right)}=1$ and $\left\|\phi_{1}\right\|_{H^{1}\left(\Omega_{1}\right)} \leqslant C_{3}$. Hence, we arrive at the following result

$$
\left\|\eta_{1}\right\|_{H\left(\mathrm{div}, \Omega_{1}\right)} \leqslant\left\|\Delta \phi_{1}\right\|_{L^{2}\left(\Omega_{1}\right)}+\left\|\Delta \phi_{2}\right\|_{L^{2}\left(\Omega_{1}\right)}+\left\|\phi_{1}\right\|_{H^{1}\left(\Omega_{1}\right)}+\left\|\phi_{2}\right\|_{H^{1}\left(\Omega_{1}\right)} \leqslant C_{4},
$$


where $C_{4}>0$ depends on $\Omega_{1}$ and $\Gamma$. Analogous results show there exists an $\eta_{\mathrm{s}}$ in $\mathbb{V}_{\mathrm{s}}$ such that

$$
-\left(\nabla \cdot \boldsymbol{\eta}_{\mathrm{s}}, \tilde{q}_{\mathrm{s}}\right)_{\Omega_{1}}+\left\langle\boldsymbol{\eta}_{\mathrm{s}} \cdot \boldsymbol{\nu}, \mu-\hat{q}_{\mathrm{s}}\right\rangle_{\Gamma}=\left\|\tilde{q}_{\mathrm{s}}\right\|_{L^{2}\left(\Omega_{\mathrm{s}}\right)}+\left\|\mu-\hat{q}_{\mathrm{s}}\right\|_{H_{\mathrm{s}}^{1 / 2}(\Gamma)}, \quad\left\|\boldsymbol{\eta}_{\mathrm{s}}\right\|_{H\left(\operatorname{div}, \Omega_{\mathrm{s}}\right)} \leqslant C_{5},
$$

where $C_{5}>0$ depends on $\Omega_{\mathrm{s}}$ and $\Gamma$.

By the definition of the $H^{-1}(\Gamma)$ norm (3.9), there exists a $\mathbf{Y}$ in $H^{1}(\Gamma)$ such that

$$
(\mathbf{Y} \cdot \boldsymbol{\nu}, \mu)_{\Gamma}=\langle\mathbf{Y}, \mu \nu\rangle_{\Gamma}=\|\mu \nu\|_{H^{-1}(\Gamma)}, \quad\|\mathbf{Y}\|_{H^{1}(\Gamma)}=1 .
$$

Taking all this together gives the result.

4.5.2 Summary. For saddle-point problems, one usually needs to only check the continuity, coercivity, and inf-sup conditions to verify well-posedness. However, there is the third bilinear form $c(\cdot, \cdot)$, whose continuity constant depends on $\Delta t^{-1}$ (see Lemma 4.8). As long as $\Delta t>0$, the system (4.14) is well-posed with a bounded solution [9, 11]. But it is important to know how the time step affects the well-posedness, especially as $\Delta t \rightarrow 0$.

The following lemma is a modification of a result in [9, Lemma 4.14], applied to our formulation, which illustrates the effect of $\Delta t$.

LEMMA 4.11 Let $\left(\boldsymbol{\eta}_{1}, \boldsymbol{\eta}_{\mathrm{s}}, \mathbf{Y}\right)$ in $\mathbb{Z}$ such that $b\left(\left(\boldsymbol{\eta}_{1}, \boldsymbol{\eta}_{\mathrm{s}}, \mathbf{Y}\right),(0,0, \mu)\right)=0$ for all $\mu \in \mathbb{M}$. Then, the bilinear forms $a$ and $b$ in (4.8), (4.9) satisfy

$$
\begin{gathered}
\frac{a\left(\left(\boldsymbol{\eta}_{1}, \boldsymbol{\eta}_{\mathrm{s}}, \mathbf{Y}\right),\left(\boldsymbol{\eta}_{\mathrm{l}}, \boldsymbol{\eta}_{\mathrm{s}}, \mathbf{Y}\right)\right)}{\left\|\left(\boldsymbol{\eta}_{\mathrm{l}}, \boldsymbol{\eta}_{\mathrm{s}}, \mathbf{Y}\right)\right\|_{\mathbb{Z}^{*}}}+\sup _{\left(q_{1}, q_{\mathrm{s}}\right) \in \mathbb{Q}_{1} \times \mathbb{Q}_{\mathrm{s}}} \frac{b\left(\left(\boldsymbol{\eta}_{\mathrm{l}}, \boldsymbol{\eta}_{\mathrm{s}}, \mathbf{Y}\right),\left(q_{1}, q_{\mathrm{s}}, 0\right)\right)}{\Delta t^{-1 / 2}\left(\left\|q_{1}\right\|_{L^{2}\left(\Omega_{1}\right)}^{2}+\left\|q_{\mathrm{s}}\right\|_{L^{2}\left(\Omega_{\mathrm{s}}\right)}^{2}\right)^{1 / 2}} \\
\geqslant C\left\|\left(\boldsymbol{\eta}_{1}, \boldsymbol{\eta}_{\mathrm{s}}, \mathbf{Y}\right)\right\|_{\mathbb{Z}^{*}},
\end{gathered}
$$

where $C>0$ depends on the physical parameters and the domain, with norm defined by

$$
\begin{aligned}
\left\|\left(\boldsymbol{\eta}_{1}, \boldsymbol{\eta}_{\mathrm{s}}, \mathbf{Y}\right)\right\|_{\mathbb{Z}^{*}}^{2}:=\frac{1}{\widehat{K}_{1}}(\| & \left.\boldsymbol{\eta}_{1}\left\|_{L^{2}\left(\Omega_{1}\right)}^{2}+\Delta t\right\| \nabla \cdot \boldsymbol{\eta}_{1} \|_{L^{2}\left(\Omega_{1}\right)}^{2}\right) \\
+ & \frac{1}{\widehat{K}_{\mathrm{s}}}\left(\left\|\boldsymbol{\eta}_{\mathrm{s}}\right\|_{L^{2}\left(\Omega_{\mathrm{s}}\right)}^{2}+\Delta t\left\|\nabla \cdot \boldsymbol{\eta}_{\mathrm{s}}\right\|_{L^{2}\left(\Omega_{\mathrm{s}}\right)}^{2}\right) \\
& +\left\|\widehat{\beta}^{-1 / 2} \mathbf{Y} \cdot \boldsymbol{\nu}\right\|_{L^{2}(\Gamma)}^{2}+\Delta t\|\mathbf{Y} \cdot \boldsymbol{\nu}\|_{H^{-1 / 2}(\Gamma)}^{2}+\Delta t \widehat{\alpha}\left\|\nabla_{\Gamma} \mathbf{Y}\right\|_{L^{2}(\Gamma)}^{2} .
\end{aligned}
$$

Lemma 4.11, and [9, Theorem 4.11, 4.13], yields the well-posedness of (4.14), but one can see more clearly how the norm is affected. An extra factor of $\Delta t$ multiplies $\left\|\nabla \cdot \eta_{1}\right\|_{L^{2}\left(\Omega_{1}\right)}^{2},\left\|\nabla \cdot \eta_{\mathrm{s}}\right\|_{L^{2}\left(\Omega_{\mathrm{s}}\right)}^{2}$, and $\|\mathbf{Y} \cdot \boldsymbol{\nu}\|_{H^{-1 / 2}(\Gamma)}^{2}$. This is reasonable given the parabolic nature of the problem. In particular, from (4.5), one can see that $\nabla \cdot \eta_{1}$ and $\nabla \cdot \eta_{\mathrm{s}}$ depends on the discrete time derivative of $u_{1}$ and $u_{\mathrm{s}}$.

\subsection{Estimates}

The semi-discrete system (4.5), (4.6) satisfies both an a priori stability bound in time and a conservation law (see the following sections). First, we note some basic results we will need. 
Proposition 4.12 Let $\mathbf{A}$ be a constant $3 \times 3$ matrix and define $\mathbf{G}(\gamma)=\mathbf{I}+\gamma \mathbf{A}$ for all $\gamma$ in $\mathbb{R}$. Then

$$
\operatorname{det} \mathbf{G}(\gamma)=1+\gamma \operatorname{trace}(\mathbf{A})+\frac{1}{2} \gamma^{2} \underbrace{\left[(\operatorname{trace}(\mathbf{A}))^{2}-\operatorname{trace}\left(\mathbf{A}^{2}\right)\right]}_{=: \Xi_{1}(\mathbf{A})}+\frac{1}{6} \gamma^{3} \Xi_{2}(\mathbf{A})
$$

where $\Xi_{1}(\mathbf{A})$ and $\Xi_{2}(\mathbf{A})$ are functions of $\mathbf{A}$ that satisfy $\left|\Xi_{1}(\mathbf{A})\right| \leqslant C|\mathbf{A}|^{2},\left|\Xi_{2}(\mathbf{A})\right| \leqslant C|\mathbf{A}|^{3}$, where $|\cdot|$ is any matrix norm and $C>0$ is a constant that only depends on the norm.

Lemma 4.13 (Discrete Grönwall Inequality) Let $c \geqslant 0$ and suppose $\left\{r_{i}\right\}_{i \geqslant 0}$ and $\left\{g_{i}\right\}_{i \geqslant 0}$ are nonnegative sequences. Then the following is true:

$$
r_{n} \leqslant c+\sum_{k=0}^{n-1} g_{k} r_{k}, \quad \text { for all } n \geqslant 0, \quad \Rightarrow \quad r_{n} \leqslant c \exp \left(\sum_{k=0}^{n-1} g_{k}\right), \quad \text { for all } n \geqslant 0,
$$

where the sum is zero when $n=0$.

4.6.1 A priori bound. We begin as we did in Section 3.4.1. Again, take $u_{\mathrm{D}}^{i+1}=0$ for $i \geqslant 0$. In (4.5) and (4.6), choose $\boldsymbol{\eta}_{1}=\sigma_{1}^{i+1}, \boldsymbol{\eta}_{\mathrm{s}}=\boldsymbol{\sigma}_{\mathrm{s}}^{i+1}, \mathbf{Y}=\mathbf{V}^{i+1}, q_{1}=-u_{1}^{i+1}, q_{\mathrm{s}}=-u_{\mathrm{s}}^{i+1}, \mu=-\lambda^{i+1}$, and add the equations together to get

$$
\begin{aligned}
\frac{1}{\widehat{K}_{1}}\left\|\sigma_{1}^{i+1}\right\|_{L^{2}\left(\Omega_{1}^{i}\right)}^{2} & +\frac{1}{\widehat{K}_{\mathrm{s}}}\left\|\sigma_{\mathrm{s}}^{i+1}\right\|_{L^{2}\left(\Omega_{\mathrm{s}}^{i}\right)}^{2}+\left\|\widehat{\beta}^{-1 / 2}\left(\nu^{i}\right) \mathbf{V}^{i+1} \cdot \nu^{i}\right\|_{L^{2}\left(\Gamma^{i}\right)}^{2} \\
& +\widehat{\alpha}\left[\left(\nabla_{\Gamma^{i}}\left(\Delta t \mathbf{V}^{i+1}\right), \nabla_{\Gamma^{i}} \mathbf{V}^{i+1}\right)_{\Gamma^{i}}+\left(\nabla_{\Gamma^{i}} \mathbf{X}^{i}, \nabla_{\Gamma^{i}} \mathbf{V}^{i+1}\right)_{\Gamma^{i}}\right] \\
& +\frac{1}{\Delta t}\left(u_{1}^{i+1},\left(u_{1}^{i+1}-\bar{u}_{1}^{i}\right)\right)_{\Omega_{1}^{i}}+\frac{1}{\Delta t}\left(u_{\mathrm{s}}^{i+1},\left(u_{\mathrm{s}}^{i+1}-{\overline{u_{\mathrm{s}}}}^{i}\right)\right)_{\Omega_{\mathrm{s}}^{i}} \\
& =\left(u_{1}^{i+1}, f_{1}^{i+1}\right)_{\Omega_{1}^{i}}+\left(u_{\mathrm{s}}^{i+1}, f_{\mathrm{s}}^{i+1}\right)_{\Omega_{\mathrm{s}}^{i}} .
\end{aligned}
$$

Next, focus on the discrete time derivative terms. Using $2 a(a-b)=a^{2}-b^{2}+(a-b)^{2}$, we obtain

$$
\left(u_{1}^{i+1},\left(u_{1}^{i+1}-\bar{u}_{1}^{i}\right)\right)_{\Omega_{1}^{i}}=\frac{1}{2}\left(\int_{\Omega_{1}^{i}}\left(u_{1}^{i+1}\right)^{2}-\int_{\Omega_{1}^{i}}\left(\bar{u}_{1}^{i}\right)^{2}+\int_{\Omega_{1}^{i}}\left(u_{1}^{i+1}-{\overline{u_{1}}}^{i}\right)^{2}\right) .
$$

Now use (4.7) and a change of variables to show

$$
\begin{aligned}
\int_{\Omega_{1}^{i}}\left({\overline{u_{1}}}^{i}\right)^{2} & =\int_{\Omega_{1}^{i-1}}\left({\overline{u_{1}}}^{i} \circ \Phi_{i}(\mathbf{x})\right)^{2} \operatorname{det}\left(\left[\nabla_{\mathbf{x}} \Phi_{i}(\mathbf{x})\right]\right) d \mathbf{x} \\
& =\int_{\Omega_{1}^{i-1}}\left(u_{1}^{i}-\Delta t \widehat{K}_{1}^{-1} \sigma_{1}^{i} \cdot \mathbf{V}^{i}\right)^{2} \operatorname{det}\left(\left[\nabla \Phi_{i}\right]\right) .
\end{aligned}
$$


Combining (4.3) and Proposition 4.12 with (4.19), and expanding, gives

$$
\begin{gathered}
\int_{\Omega_{1}^{i}}\left(\bar{u}_{1}^{i}\right)^{2} \leqslant \int_{\Omega_{1}^{i-1}}\left(\left(u_{1}^{i}\right)^{2}+2 \Delta t \widehat{K}_{1}^{-1}\left|u_{1}^{i} \| \sigma_{1}^{i}\right|\left|\mathbf{V}^{i}\right|+\Delta t^{2} \widehat{K}_{1}^{-2}\left|\sigma_{1}^{i}\right|^{2}\left|\mathbf{V}^{i}\right|^{2}\right) . \\
\cdot\left(1+\Delta t\left|\nabla \cdot \mathbf{V}^{i}\right|+\left(\Delta t^{2} / 2\right)\left|\Xi_{1}\left(\nabla \mathbf{V}^{i}\right)\right|+\left(\Delta t^{3} / 6\right)\left|\Xi_{2}\left(\nabla \mathbf{V}^{i}\right)\right|\right) d \mathbf{x} \\
\leqslant\left\|u_{1}^{i}\right\|_{L^{2}\left(\Omega_{1}^{i-1}\right)}^{2}+M_{1, i} \Delta t\left(\frac{2}{\widehat{K}_{1}}\left\|u_{1}^{i}\right\|_{L^{2}\left(\Omega_{1}^{i-1}\right)}\left\|\sigma_{1}^{i}\right\|_{L^{2}\left(\Omega_{1}^{i-1}\right)}+\left\|u_{1}^{i}\right\|_{L^{2}\left(\Omega_{1}^{i-1}\right)}^{2}\right) \\
+\left(M_{1, i}^{2} \Delta t^{2}+M_{1, i}^{3} \Delta t^{3}\right) \\
\cdot\left(\frac{1}{\widehat{K}_{1}^{2}}\left\|\sigma_{1}^{i}\right\|_{L^{2}\left(\Omega_{1}^{i-1}\right)}^{2}+\frac{2 C}{\widehat{K}_{1}}\left\|u_{1}^{i}\right\|_{L^{2}\left(\Omega_{1}^{i-1}\right)}\left\|\sigma_{1}^{i}\right\|_{L^{2}\left(\Omega_{1}^{i-1}\right)}+C\left\|u_{1}^{i}\right\|_{L^{2}\left(\Omega_{1}^{i-1}\right)}^{2}\right) \\
+C M_{1, i}^{4} \Delta t^{4}\left(\frac{1}{\widehat{K}_{1}^{2}}\left\|\sigma_{1}^{i}\right\|_{L^{2}\left(\Omega_{1}^{i-1}\right)}^{2}+\frac{2}{\widehat{K}_{1}}\left\|u_{1}^{i}\right\|_{L^{2}\left(\Omega_{1}^{i-1}\right)}\left\|\sigma_{1}^{i}\right\|_{L^{2}\left(\Omega_{1}^{i-1}\right)}\right) \\
+C M_{1, i}^{5} \Delta t^{5} \frac{1}{\widehat{K}_{1}^{2}}\left\|\sigma_{1}^{i}\right\|_{L^{2}\left(\Omega_{1}^{i-1}\right)}^{2} \\
+\left\|u_{1}^{i}\right\|_{L^{2}\left(\Omega_{1}^{i-1}\right)}^{2 \Delta t} \frac{M_{1, i}}{\widehat{K}_{1}}\left\|u_{1}^{i}\right\|_{L^{2}\left(\Omega_{1}^{i-1}\right)}\left\|\sigma_{1}^{i}\right\|_{L^{2}\left(\Omega_{1}^{i-1}\right)}\left(1+C M_{1, i} \Delta t+C M_{1, i}^{2} \Delta t^{2}+C M_{1, i}^{3} \Delta t^{3}\right) \\
\left.+\Delta t M_{1, i}\left\|u_{1}^{i}\right\|_{L^{2}\left(\Omega_{1}^{2}\right.}^{2}\right) \\
+\Delta t^{2} \frac{M_{1, i}^{2}}{\widehat{K}_{1}^{2}}\left\|\sigma_{1}^{i}\right\|_{L^{2}\left(\Omega_{1}^{i-1}\right)}^{2}\left(1+C M_{1, i} \Delta t+C M_{1, i}^{2} \Delta t^{2}\right)
\end{gathered}
$$

where $M_{1, i}=\left\|\mathbf{V}^{i}\right\|_{W^{1, \infty}\left(\Omega_{1}^{i-1}\right)}$ and $C>0$ is an independent constant. Next, choose $\Delta t$ such that

$$
M_{1, i} \Delta t, C M_{1, i} \Delta t, C M_{1, i}^{2} \Delta t^{2}, C M_{1, i}^{3} \Delta t^{3} \leqslant 1 / 3, \quad \Delta t M_{1, i}^{2} / \widehat{K}_{1} \leqslant 1 / 4
$$

and note the following weighted Young's inequality:

$$
\frac{\Delta t}{\widehat{K}_{1}} M_{1, i} 2\left\|u_{1}^{i}\right\|_{L^{2}\left(\Omega_{1}^{i-1}\right)}\left\|\sigma_{1}^{i}\right\|_{L^{2}\left(\Omega_{1}^{i-1}\right)} \leqslant \frac{\Delta t}{\widehat{K}_{1}}\left(4 M_{1, i}^{2}\left\|u_{1}^{i}\right\|_{L^{2}\left(\Omega_{1}^{i-1}\right)}^{2}+\frac{1}{4}\left\|\sigma_{1}^{i}\right\|_{L^{2}\left(\Omega_{1}^{i-1}\right)}^{2}\right) .
$$

Hence, (4.20) implies

$$
\int_{\Omega_{1}^{i}}\left({\overline{u_{1}}}^{i}\right)^{2} \leqslant\left\|u_{1}^{i}\right\|_{L^{2}\left(\Omega_{1}^{i-1}\right)}^{2}+\Delta t \underbrace{\left(\frac{8 M_{1, i}^{2}}{\widehat{K}_{1}}+2 M_{1, i}\right)}_{=: \widehat{M}_{1, i}}\left\|u_{1}^{i}\right\|_{L^{2}\left(\Omega_{1}^{i-1}\right)}^{2}+\frac{\Delta t}{\widehat{K}_{1}}\left\|\sigma_{1}^{i}\right\|_{L^{2}\left(\Omega_{1}^{i-1}\right)}^{2},
$$

A similar result holds for $u_{\mathrm{s}}^{i}$, with constant $\widetilde{M_{\mathrm{s}, i}}=\left(8 M_{\mathrm{s}, i}^{2} / \widehat{K_{\mathrm{s}}}\right)+2 M_{\mathrm{s}, i}$ where $M_{\mathrm{s}, i}=$ $\left\|\mathbf{V}^{i}\right\|_{W^{1, \infty\left(\Omega_{\mathrm{s}}^{i-1}\right)}}$. 
Next, we note a result from [3, Lemma 1] which says that

$$
\int_{\Gamma^{i}} \nabla_{\Gamma} \mathbf{X}^{i+1} \cdot \nabla_{\Gamma}\left(\mathbf{X}^{i+1}-\mathbf{X}^{i}\right) \geqslant\left|\mathbf{X}^{i+1}\left(\Gamma^{i}\right)\right|-\left|\Gamma^{i}\right|=\left|\Gamma^{i+1}\right|-\left|\Gamma^{i}\right|
$$

where $\Gamma^{i+1}:=\mathbf{X}^{i+1}\left(\Gamma^{i}\right)$. Hence,

$$
\begin{aligned}
\left(\nabla_{\Gamma^{i}}\left(\Delta t \mathbf{V}^{i+1}\right), \nabla_{\Gamma^{i}} \mathbf{V}^{i+1}\right)_{\Gamma^{i}}+\left(\nabla_{\Gamma^{i}} \mathbf{X}^{i}, \nabla_{\Gamma^{i}} \mathbf{V}^{i+1}\right)_{\Gamma^{i}} & =\Delta t^{-1}\left(\nabla_{\Gamma^{i}} \mathbf{X}^{i+1}, \nabla_{\Gamma^{i}}\left(\mathbf{X}^{i+1}-\mathbf{X}^{i}\right)\right)_{\Gamma^{i}} \\
& \geqslant \frac{\left|\Gamma^{i+1}\right|-\left|\Gamma^{i}\right|}{\Delta t} .
\end{aligned}
$$

Now combine (4.22) with (4.18) and plug into (4.17) (and do the same for $u_{\mathrm{s}}^{i}$ ). Using (4.23) then yields

$$
\begin{aligned}
& \frac{1}{\widehat{K}_{1}}\left\|\sigma_{1}^{i+1}\right\|_{L^{2}\left(\Omega_{1}^{i}\right)}^{2}-\frac{1}{2} \frac{1}{\widehat{K}_{1}}\left\|\sigma_{1}^{i}\right\|_{L^{2}\left(\Omega_{1}^{i-1}\right)}^{2}+\frac{1}{\widehat{K}_{\mathrm{s}}}\left\|\sigma_{\mathrm{s}}^{i+1}\right\|_{L^{2}\left(\Omega_{\mathrm{s}}^{i}\right)}^{2}-\frac{1}{2} \frac{1}{\widehat{K}_{\mathrm{s}}}\left\|\sigma_{\mathrm{s}}^{i}\right\|_{L^{2}\left(\Omega_{\mathrm{s}}^{i-1}\right)}^{2} \\
& +\left\|\widehat{\beta}^{-1 / 2}\left(\nu^{i}\right) \mathbf{V}^{i+1} \cdot \nu^{i}\right\|_{L^{2}\left(\Gamma^{i}\right)}^{2}+\widehat{\alpha} \frac{\left|\Gamma^{i+1}\right|-\left|\Gamma^{i}\right|}{\Delta t}+\frac{1}{\Delta t} \frac{1}{2}\left(\left\|u_{1}^{i+1}\right\|_{L^{2}\left(\Omega_{1}^{i}\right)}^{2}-\left\|u_{1}^{i}\right\|_{L^{2}\left(\Omega_{1}^{i-1}\right)}^{2}\right) \\
& +\frac{1}{\Delta t} \frac{1}{2}\left(\left\|u_{\mathrm{s}}^{i+1}\right\|_{L^{2}\left(\Omega_{\mathrm{s}}^{i}\right)}^{2}-\left\|u_{\mathrm{s}}^{i}\right\|_{L^{2}\left(\Omega_{\mathrm{s}}^{i-1}\right)}^{2}\right) \\
& \leqslant\left(u_{1}^{i+1}, f_{1}^{i+1}\right)_{\Omega_{1}^{i}}+\frac{1}{2} \widetilde{M_{1, i}}\left\|u_{1}^{i}\right\|_{L^{2}\left(\Omega_{1}^{i-1}\right)}^{2}+\left(u_{\mathrm{s}}^{i+1}, f_{\mathrm{s}}^{i+1}\right)_{\Omega_{\mathrm{s}}^{i}}+\frac{1}{2} \widetilde{M_{\mathrm{s}, i}}\left\|u_{\mathrm{s}}^{i}\right\|_{L^{2}\left(\Omega_{\mathrm{s}}^{i-1}\right)}^{2}
\end{aligned}
$$

Applying a weighted Young's inequality to the right-hand-side, multiplying by $\Delta t$, summing over $i$, and cancelling similar terms, we get

$$
\begin{aligned}
& \frac{\Delta t}{2} \sum_{i=0}^{N-1}\left[\frac{1}{\widehat{K}_{1}}\left\|\sigma_{1}^{i+1}\right\|_{L^{2}\left(\Omega_{1}^{i}\right)}^{2}+\frac{1}{\widehat{K}_{\mathrm{s}}}\left\|\sigma_{\mathrm{s}}^{i+1}\right\|_{L^{2}\left(\Omega_{\mathrm{s}}^{i}\right)}^{2}\right]+\Delta t \sum_{i=0}^{N-1}\left\|\widehat{\beta}^{-1 / 2}\left(\nu^{i}\right) \mathbf{V}^{i+1} \cdot \nu^{i}\right\|_{L^{2}\left(\Gamma^{i}\right)}^{2} \\
& \quad+\widehat{\alpha}\left|\Gamma^{N}\right|+\frac{1}{2}\left\|u_{1}^{N}\right\|_{L^{2}\left(\Omega_{1}^{N-1}\right)}^{2}+\frac{1}{2}\left\|u_{\mathrm{s}}^{N}\right\|_{L^{2}\left(\Omega_{\mathrm{s}}^{N-1}\right)}^{2} \\
& \leqslant \\
& \quad \widehat{\alpha}\left|\Gamma^{0}\right|+\frac{1}{2}\left(\frac{\Delta t}{\widehat{K}_{1}}\left\|\sigma_{1}^{0}\right\|_{L^{2}\left(\Omega_{1}^{-1}\right)}^{2}+\frac{\Delta t}{\widehat{K}_{\mathrm{s}}}\left\|\sigma_{\mathrm{s}}^{0}\right\|_{L^{2}\left(\Omega_{\mathrm{s}}^{-1}\right)}^{2}+\left\|u_{1}^{0}\right\|_{L^{2}\left(\Omega_{1}^{-1}\right)}^{2}+\left\|u_{\mathrm{s}}^{0}\right\|_{L^{2}\left(\Omega_{\mathrm{s}}^{-1}\right)}^{2}\right) \\
& \quad+\Delta t \sum_{i=0}^{N-1}\left[\left\|f_{1}^{i+1}\right\|_{L^{2}\left(\Omega_{1}^{i}\right)}^{2}+\left\|f_{\mathrm{s}}^{i+1}\right\|_{L^{2}\left(\Omega_{\mathrm{s}}^{i}\right)}^{2}\right] \\
& \quad+\frac{\Delta t}{2} \sum_{i=0}^{N-1}\left[\frac{1}{2}\left\|u_{1}^{i+1}\right\|_{L^{2}\left(\Omega_{1}^{i}\right)}^{2}+\widetilde{M_{1, i}}\left\|u_{1}^{i}\right\|_{L^{2}\left(\Omega_{1}^{i-1}\right)}^{2}+\frac{1}{2}\left\|u_{\mathrm{s}}^{i+1}\right\|_{L^{2}\left(\Omega_{\mathrm{s}}^{i}\right)}^{2}+\widetilde{M_{\mathrm{s}, i}}\left\|_{u_{\mathrm{s}}^{i}}\right\|_{L^{2}\left(\Omega_{\mathrm{s}}^{i-1}\right)}^{2}\right]
\end{aligned}
$$

where $N$ is the last time index to compute. Making further simplifications, and assuming $\Delta t \leqslant 1$, 
we arrive at

$$
\begin{gathered}
\frac{\Delta t}{2} \sum_{i=0}^{N-1}\left[\frac{1}{\widehat{K}_{1}}\left\|\sigma_{1}^{i+1}\right\|_{L^{2}\left(\Omega_{1}^{i}\right)}^{2}+\frac{1}{\widehat{K}_{\mathrm{s}}}\left\|\sigma_{\mathrm{s}}^{i+1}\right\|_{L^{2}\left(\Omega_{\mathrm{s}}^{i}\right)}^{2}\right]+\Delta t \sum_{i=0}^{N-1}\left\|\widehat{\beta}^{-1 / 2}\left(\nu^{i}\right) \mathbf{V}^{i+1} \cdot \nu^{i}\right\|_{L^{2}\left(\Gamma^{i}\right)}^{2} \\
\quad+\widehat{\alpha}\left|\Gamma^{N}\right|+\frac{1}{4}\left\|u_{1}^{N}\right\|_{L^{2}\left(\Omega_{1}^{N-1}\right)}^{2}+\frac{1}{4}\left\|u_{\mathrm{s}}^{N}\right\|_{L^{2}\left(\Omega_{\mathrm{s}}^{N-1}\right)}^{2} \\
\leqslant \widehat{\alpha}\left|\Gamma^{0}\right|+\frac{1}{2}\left(\frac{\Delta t}{\widehat{K}_{1}}\left\|\sigma_{1}^{0}\right\|_{L^{2}\left(\Omega_{1}^{-1}\right)}^{2}+\frac{\Delta t}{\widehat{K}_{\mathrm{s}}}\left\|\sigma_{\mathrm{s}}^{0}\right\|_{L^{2}\left(\Omega_{\mathrm{s}}^{-1}\right)}^{2}+\left\|u_{1}^{0}\right\|_{L^{2}\left(\Omega_{1}^{-1}\right)}^{2}+\left\|u_{\mathrm{s}}^{0}\right\|_{L^{2}\left(\Omega_{\mathrm{s}}^{-1}\right)}^{2}\right) \\
+\Delta t \sum_{i=0}^{N-1}\left[\left\|f_{1}^{i+1}\right\|_{L^{2}\left(\Omega_{1}^{i}\right)}^{2}+\left\|f_{\mathrm{s}}^{i+1}\right\|_{L^{2}\left(\Omega_{\mathrm{s}}^{i}\right)}^{2}\right] \\
+\frac{\Delta t}{2} \sum_{i=0}^{N-1} v_{i}\left[\left\|u_{1}^{i}\right\|_{L^{2}\left(\Omega_{1}^{i-1}\right)}^{2}+\left\|u_{\mathrm{s}}^{i}\right\|_{L^{2}\left(\Omega_{\mathrm{s}}^{i-1}\right)}^{2}\right]
\end{gathered}
$$

where $V_{i}>0$ is a constant that depends on $\left\|\mathbf{V}^{i}\right\|_{W^{1, \infty}(\Omega)}$. Note that $u_{1}^{0}\left(u_{\mathrm{s}}^{0}\right)$ is the initial temperature on the initial domain $\Omega_{1}^{-1}\left(\Omega_{\mathrm{s}}^{-1}\right)$; similar for the flux $\sigma_{1}^{0}\left(\sigma_{\mathrm{s}}^{0}\right)$.

Applying Lemma 4.13 to (4.25) yields

$$
\frac{1}{4}\left[\left\|u_{1}^{k}\right\|_{L^{2}\left(\Omega_{1}^{k-1}\right)}^{2}+\left\|u_{\mathrm{s}}^{k}\right\|_{L^{2}\left(\Omega_{\mathrm{s}}^{k-1}\right)}^{2}\right] \leqslant A_{0} \exp \left(2 \Delta t \sum_{p=0}^{k-1} \mho_{p}\right), \quad \text { for all } k \geqslant 0
$$

where $A_{0}$ is given in Theorem 4.14, which we have now proved.

THEOREM 4.14 Suppose (4.5), (4.6), (4.7) is solved on $\Omega^{i}$ at time index $i$, with $u_{\mathrm{D}}^{i+1}=0$, and assume $\mathbf{V}^{i+1}$ is in $W^{1, \infty}\left(\Omega^{i}\right)$ and that $\Phi_{i+1}$ is a bijective map in $W^{1, \infty}\left(\Omega^{i}\right)$ with bounded inverse. Suppose this holds for $i=0, \ldots, N-1$. If $\Delta t \leqslant 1$ also satisfies

$$
\Delta t \leqslant \frac{B_{0}}{\max _{0 \leqslant i \leqslant N}\left\|\mathbf{V}^{i}\right\|_{W^{1, \infty}(\Omega)}} \quad \text { and } \quad \Delta t \leqslant B_{0} \frac{\max \left(\widehat{K}_{1}, \widehat{K}_{\mathrm{s}}\right)}{\max _{0 \leqslant i \leqslant N}\left\|\mathbf{V}^{i}\right\|_{W^{1, \infty}(\Omega)}^{2}}
$$

for some independent constant $B_{0}>0$, then

$$
\begin{array}{r}
\frac{\Delta t}{2} \sum_{i=0}^{N-1}\left[\frac{1}{\widehat{K}_{1}}\left\|\sigma_{1}^{i+1}\right\|_{L^{2}\left(\Omega_{1}^{i}\right)}^{2}+\frac{1}{\widehat{K}_{\mathrm{s}}}\left\|\boldsymbol{\sigma}_{\mathrm{s}}^{i+1}\right\|_{L^{2}\left(\Omega_{\mathrm{s}}^{i}\right)}^{2}\right]+\Delta t \sum_{i=0}^{N-1}\left\|\widehat{\beta}^{-1 / 2}\left(\boldsymbol{\nu}^{i}\right) \mathbf{V}^{i+1} \cdot \nu^{i}\right\|_{L^{2}\left(\Gamma^{i}\right)}^{2} \\
+\widehat{\alpha}\left|\Gamma^{N}\right|+\frac{1}{4}\left\|u_{1}^{N}\right\|_{L^{2}\left(\Omega_{1}^{N-1}\right)}^{2}+\frac{1}{4}\left\|u_{\mathrm{s}}^{N}\right\|_{L^{2}\left(\Omega_{\mathrm{s}}^{N-1}\right)}^{2} \\
\leqslant A_{0}+A_{0} 2 \Delta t \sum_{i=0}^{N-1} \vartheta_{i} \exp \left(2 \Delta t \sum_{p=0}^{i-1} \vartheta_{p}\right)
\end{array}
$$


where

$$
\begin{aligned}
& A_{0}=\widehat{\alpha} \mid \Gamma^{0} \mid+\frac{1}{2}\left(\frac{\Delta t}{\widehat{K}_{1}}\left\|\sigma_{1}^{0}\right\|_{L^{2}\left(\Omega_{1}^{-1}\right)}^{2}+\frac{\Delta t}{\widehat{K}_{\mathrm{s}}}\left\|\sigma_{\mathrm{s}}^{0}\right\|_{L^{2}\left(\Omega_{\mathrm{s}}^{-1}\right)}^{2}+\left\|u_{1}^{0}\right\|_{L^{2}\left(\Omega_{1}^{-1}\right)}^{2}+\left\|u_{\mathrm{s}}^{0}\right\|_{L^{2}\left(\Omega_{\mathrm{s}}^{-1}\right)}^{2}\right) \\
&+\Delta t \sum_{i=0}^{N-1}\left[\left\|f_{1}^{i+1}\right\|_{L^{2}\left(\Omega_{1}^{i}\right)}^{2}+\left\|f_{\mathrm{s}}^{i+1}\right\|_{L^{2}\left(\Omega_{\mathrm{s}}^{i}\right)}^{2}\right],
\end{aligned}
$$

where $V_{i}>0$ is a constant that depends on $\left\|\mathbf{V}^{i}\right\|_{W^{1, \infty}(\Omega)}$. Note: the final time for the semi-discrete evolution is $T=\Delta t N$.

REMARK 4.15 Using an $L^{2}$ projection for the temperatures from one time step to the next would give a better estimate (i.e. more in-line with the fully continuous result (3.20)). The approach taken here is more complicated because we introduced the material derivative with an explicit treatment of the convective term (recall (4.4) and (4.7)); see Remark 5.8 for the reason. Theorem 4.14 can be easily modified to allow $u_{\mathrm{D}}^{i+1} \neq 0$.

4.6.2 Conservation law. Analogous to Section 3.4.2, choose $q_{1}=1, q_{\mathrm{s}}=1$ in (4.5), and $\mu=1$ in (4.6) to get

$$
\begin{aligned}
-\int_{\partial_{\mathrm{D}} \Omega} \sigma_{1}^{i+1} \cdot \nu_{\Omega} & +\int_{\Gamma^{i}} \sigma_{1}^{i+1} \cdot \nu^{i}=\frac{1}{\Delta t}\left(\int_{\Omega_{1}^{i}} u_{1}^{i+1}-\int_{\Omega_{1}^{i}} \bar{u}_{1}^{i}\right)-\int_{\Omega_{1}^{i}} f_{1}^{i+1}, \\
- & \int_{\Gamma^{i}} \sigma_{\mathrm{s}}^{i+1} \cdot \nu^{i}=\frac{1}{\Delta t}\left(\int_{\Omega_{\mathrm{s}}^{i}} u_{\mathrm{s}}^{i+1}-\int_{\Omega_{\mathrm{s}}^{i}}{\overline{u_{\mathrm{s}}}}^{i}\right)-\int_{\Omega_{\mathrm{s}}^{i}} f_{\mathrm{s}}^{i+1}, \\
& \widehat{S} \int_{\Gamma^{i}} \mathbf{V}^{i+1} \cdot \boldsymbol{\nu}^{i}=\int_{\Gamma^{i}} \sigma_{1}^{i+1} \cdot \boldsymbol{\nu}^{i}-\int_{\Gamma^{i}} \boldsymbol{\sigma}_{\mathrm{s}}^{i+1} \cdot \boldsymbol{\nu}^{i} .
\end{aligned}
$$

Just as in (4.19), we have

$$
\int_{\Omega_{1}^{i}}{\overline{u_{1}}}^{i}=\int_{\Omega_{1}^{i-1}}{\overline{u_{1}}}^{i} \circ \Phi_{i}(\mathbf{x}) \operatorname{det}\left(\left[\nabla_{\mathbf{x}} \Phi_{i}(\mathbf{x})\right]\right) d \mathbf{x}=\int_{\Omega_{1}^{i-1}}\left(u_{1}^{i}-\Delta t \widehat{K}_{1}^{-1} \sigma_{1}^{i} \cdot \mathbf{V}^{i}\right) \operatorname{det}\left(\left[\nabla \Phi_{i}\right]\right) .
$$

Combining (4.3) and Proposition 4.12 with (4.29), and expanding, gives

$$
\begin{aligned}
\int_{\Omega_{1}^{i}}{\overline{u_{1}}}^{i} & =\int_{\Omega_{1}^{i-1}}\left(u_{1}^{i}-\Delta t \widehat{K}_{1}^{-1} \sigma_{1}^{i} \cdot \mathbf{V}^{i}\right) \\
& \left.=\int_{\Omega_{1}^{i-1}} u_{1}^{i}+\Delta t \int_{\Omega_{1}^{i-1}}\left(1+\Delta t \nabla \cdot \mathbf{V}_{1}^{i} \nabla \cdot\left(\Delta t^{2} / 2\right) \mathbf{V}_{1}\left(\nabla \mathbf{V}^{i}\right)+\left(\Delta t^{3} / 6\right) \widehat{K}_{1}-1 \sigma_{1}^{i} \cdot \mathbf{V}^{i}\right)\right)+\Delta t^{2} I_{1}
\end{aligned}
$$

where $I_{1}$ contains the higher order terms (c.f. (4.20)):

$$
\begin{aligned}
I_{1}= & \int_{\Omega_{1}^{i-1}}\left\{\left(u_{1}^{i} / 2\right) \Xi_{1}\left(\nabla \mathbf{V}^{i}\right)-\widehat{K}_{1}^{-1} \sigma_{1}^{i} \cdot \mathbf{V}^{i}\left(\nabla \cdot \mathbf{V}^{i}\right)\right. \\
& \left.+\Delta t\left[\left(u_{1}^{i} / 6\right) \Xi_{2}\left(\nabla \mathbf{V}^{i}\right)-(1 / 2) \widehat{K}_{1}^{-1} \sigma_{1}^{i} \cdot \mathbf{V}^{i} \Xi_{1}\left(\nabla \mathbf{V}^{i}\right)\right]-\Delta t^{2}(1 / 6) \widehat{K}_{1}^{-1} \sigma_{1}^{i} \cdot \mathbf{V}^{i} \Xi_{2}\left(\nabla \mathbf{V}^{i}\right)\right\}
\end{aligned}
$$


Next, by making judicious use of the weak formulation (4.5), we can simplify the $O(\Delta t)$ term in (4.30). Choose $\eta_{1}=\left.\mathbf{V}^{i}\right|_{\Omega_{1}} \in \mathbb{V}_{1}^{i-1}(0)$ in (4.5) on $\Omega_{1}^{i-1}$ for any $i \geqslant 1$, and note (4.2):

$$
\int_{\Omega_{1}^{i-1}} \widehat{K}_{1}^{-1} \boldsymbol{\sigma}_{1}^{i} \cdot \mathbf{V}^{i}-\int_{\Omega_{1}^{i-1}} u_{1}^{i} \nabla \cdot \mathbf{V}^{i}=\int_{\Gamma^{i-1}} \lambda^{i} \mathbf{V}^{i} \cdot \nu^{i-1}
$$

Thus, we arrive at

$$
\begin{aligned}
\int_{\Omega_{1}^{i}}{\overline{u_{1}}}^{i} & =\int_{\Omega_{1}^{i-1}} u_{1}^{i}-\Delta t \int_{\Gamma^{i-1}} \lambda^{i} \mathbf{V}^{i} \cdot \nu^{i-1}+\Delta t^{2} I_{1}, \quad \text { for all } i \geqslant 1, \\
\left|I_{1}\right| & \leqslant C_{0} M_{1, i}^{2}\left[\left\|u_{1}^{i}\right\|_{L^{1}\left(\Omega_{1}^{i-1}\right)}\left(1+\Delta t M_{1, i}\right)+\widehat{K}_{1}^{-1}\left\|\sigma_{1}^{i}\right\|_{L^{1}\left(\Omega_{1}^{i-1}\right)}\left(1+\Delta t M_{1, i}+\Delta t^{2} M_{1, i}^{2}\right)\right],
\end{aligned}
$$

where $M_{1, i}=\left\|\mathbf{V}^{i}\right\|_{W^{1, \infty}\left(\Omega_{1}^{i-1}\right)}$ and $C_{0}>0$ is an independent constant. Note that setting the initial velocity $\mathbf{V}^{0}=\mathbf{0}$ gives $\int_{\Omega_{1}^{0}}{\overline{u_{1}}}^{0} \equiv \int_{\Omega_{1}^{-1}} u_{1}^{0}$. Similar results hold for $u_{\mathrm{s}}^{i}$ :

$$
\begin{aligned}
\int_{\Omega_{\mathrm{s}}^{i}}{\overline{u_{\mathrm{s}}}}^{i} & =\int_{\Omega_{\mathrm{s}}^{i-1}} u_{\mathrm{s}}^{i}+\Delta t \int_{\Gamma^{i-1}} \lambda^{i} \mathbf{V}^{i} \cdot \nu^{i-1}+\Delta t^{2} I_{\mathrm{s}}, \quad \text { for all } i \geqslant 1, \\
\left|I_{\mathrm{s}}\right| & \leqslant C_{0} M_{\mathrm{s}, i}^{2}\left[\left\|u_{\mathrm{s}}^{i}\right\|_{L^{1}\left(\Omega_{\mathrm{s}}^{i-1}\right)}\left(1+\Delta t M_{\mathrm{s}, i}\right)+\widehat{K}_{\mathrm{s}}^{-1}\left\|\sigma_{\mathrm{s}}^{i}\right\|_{L^{1}\left(\Omega_{\mathrm{s}}^{i-1}\right)}\left(1+\Delta t M_{\mathrm{s}, i}+\Delta t^{2} M_{\mathrm{s}, i}^{2}\right)\right],
\end{aligned}
$$

where $M_{\mathrm{s}, i}=\left\|\mathbf{V}^{i}\right\|_{W^{1, \infty}\left(\Omega_{\mathrm{s}}^{i-1}\right)}$.

Therefore, adding the equations in (4.28), and using (4.32) and (4.33), gives a time-discrete thermal power balance for each $i=0, \ldots, N-1$ :

$$
\begin{array}{r}
\int_{\Omega_{1}^{i}} f_{1}^{i+1}+\int_{\Omega_{\mathrm{s}}^{i}} f_{\mathrm{s}}^{i+1}-\int_{\partial_{\mathrm{D}} \Omega} \sigma_{1}^{i+1} \cdot \nu_{\Omega}=\frac{1}{\Delta t}\left(\int_{\Omega_{1}^{i}} u_{1}^{i+1}-\int_{\Omega_{1}^{i-1}} u_{1}^{i}\right) \\
+\frac{1}{\Delta t}\left(\int_{\Omega_{\mathrm{s}}^{i}} u_{\mathrm{s}}^{i+1}-\int_{\Omega_{\mathrm{s}}^{i-1}} u_{\mathrm{s}}^{i}\right)-\widehat{S} \int_{\Gamma^{i}} \mathbf{V}^{i+1} \cdot \nu^{i}-\Delta t\left(I_{1}+I_{\mathrm{s}}\right) .
\end{array}
$$

Finally, summing (4.34) over the time steps, and bounding $I_{1}$ and $I_{\mathrm{s}}$, yields the following theorem.

THEOREM 4.16 Assume the hypothesis of Theorem 4.14 and suppose $\mathbf{V}^{0} \equiv \mathbf{0}$ on $\Omega$. Then,

$$
\begin{gathered}
\mid \Delta t \sum_{i=0}^{N-1}\left(\int_{\Omega_{1}^{i}} f_{1}^{i+1}+\int_{\Omega_{\mathrm{s}}^{i}} f_{\mathrm{s}}^{i+1}-\int_{\partial_{\mathrm{D}} \Omega} \sigma_{1}^{i+1} \cdot \nu_{\Omega}\right)+\int_{\Omega_{1}^{-1}} u_{1}^{0}+\int_{\Omega_{\mathrm{s}}^{-1}} u_{\mathrm{s}}^{0} \\
\quad-\int_{\Omega_{1}^{N-1}} u_{1}^{N}-\int_{\Omega_{\mathrm{s}}^{N-1}} u_{\mathrm{s}}^{N}+\Delta t \widehat{S} \sum_{i=0}^{N-1} \int_{\Gamma^{i}} \mathbf{V}^{i+1} \cdot \nu^{i} \mid \leqslant \Delta t B_{1},
\end{gathered}
$$

where

$$
B_{1}=\Delta t \sum_{i=0}^{N-1} V_{i}\left[\frac{1}{\widehat{K}_{1}}\left\|\sigma_{1}^{i}\right\|_{L^{1}\left(\Omega_{1}^{i-1}\right)}+\frac{1}{\widehat{K}_{\mathrm{s}}}\left\|\sigma_{\mathrm{s}}^{i}\right\|_{L^{1}\left(\Omega_{\mathrm{s}}^{i-1}\right)}+\left\|u_{1}^{i}\right\|_{L^{1}\left(\Omega_{1}^{i-1}\right)}+\left\|u_{\mathrm{s}}^{i}\right\|_{L^{1}\left(\Omega_{\mathrm{s}}^{i-1}\right)}\right],
$$


and $V_{i}>0$ is a constant that depends on $\left\|\mathbf{V}^{i}\right\|_{W^{1, \infty}(\Omega)}$. Note that $B_{1}$ is uniformly bounded (with respect to $\Delta t$ ) by Theorem 4.14 .

REMARK 4.17 Theorem 4.16 is a discrete time integral version of (3.21), except with an $O(\Delta t)$ error. The conservation property in [5, Remark 3.5] is exact but only applies to a time-continuous version of their numerical scheme. We note that Theorem 4.16 can be modified to allow $u_{\mathrm{D}}^{i+1} \neq 0$ and $\mathbf{V}^{0} \neq \mathbf{0}$.

\section{Fully discrete formulation}

\subsection{Discretization}

5.1.1 Non-degenerate interface. Let $\nu_{h}$ denote the unit normal vector on $\Gamma_{h}$ and $\partial \Omega_{h}$. The following assumption is the space discrete version of Assumption 4.5 in Section 4.4.1. It is necessary to ensure the equivalence of the norms in the space discrete version of Proposition 4.6 when $\|\cdot\|_{H^{-1 / 2}}$ is replaced by a discrete norm $\|\cdot\|_{H_{h}^{-1 / 2}}$.

Assumption 5.1 Assume that $\Gamma_{h}$ is a polyhedral manifold (i.e. a surface triangulation). For any vertex $v$, let $\operatorname{Star}(v)$ be the set of triangle faces in $\Gamma_{h}$ that contain $v$ as a vertex. For any non-zero constant vector $\mathbf{a} \in \mathbb{R}^{3}$, assume there exists a vertex $v$ in $\Gamma_{h}$ such that $|\operatorname{Star}(v)| \geqslant c_{0}>0$ and

$$
\mathbf{a} \cdot \boldsymbol{\nu}_{h}(\mathbf{x})>0, \quad \forall \mathbf{x} \in \operatorname{Star}(v), \quad \text { or } \mathbf{a} \cdot \boldsymbol{\nu}_{h}(\mathbf{x})<0, \quad \forall \mathbf{x} \in \operatorname{Star}(v)
$$

5.1.2 Formulation. We begin by approximating the domains $\Omega_{1}^{i}, \Omega_{\mathrm{s}}^{i}$ by three dimensional triangulations $\Omega_{1, h}^{i}, \Omega_{\mathrm{s}, h}^{i}$ such that $\Gamma_{h}^{i}=\overline{\Omega_{1, h}^{i}} \cap \overline{\Omega_{\mathrm{s}, h}^{i}}$ is an embedded polyhedral surface contained in the faces of the mesh. A standard Galerkin approximation of equations (4.5), (4.6) takes the form: find $\sigma_{1, h}^{i+1}$ in $\mathbb{V}_{1, h}^{i}(0) \subset \mathbb{V}_{1}^{i}(0), \sigma_{\mathrm{s}, h}^{i+1}$ in $\mathbb{V}_{\mathrm{s}, h}^{i} \subset \mathbb{V}_{\mathrm{s}}^{i}, \mathbf{V}_{h}^{i+1}$ in $\mathbb{Y}_{h}^{i} \subset \mathbb{Y}^{i}, u_{1, h}^{i+1}$ in $\mathbb{Q}_{1, h}^{i} \subset \mathbb{Q}_{1}^{i}, u_{\mathrm{s}, h}^{i+1}$ in $\mathbb{Q}_{\mathrm{s}, h}^{i} \subset \mathbb{Q}_{\mathrm{s}}^{i}, \lambda_{h}^{i+1}$ in $\mathbb{M}_{h}^{i} \subset \mathbb{M}^{i}$ such that for all $\boldsymbol{\eta}_{\mathrm{l}} \in \mathbb{V}_{1, h}^{i}(0), \boldsymbol{\eta}_{\mathrm{s}} \in \mathbb{V}_{\mathrm{s}, h}^{i}, q_{1} \in \mathbb{Q}_{1, h}^{i}, q_{\mathrm{s}} \in \mathbb{Q}_{\mathrm{s}, h}^{i}$, $\mathbf{Y} \in \mathbb{Y}_{h}^{i}, \mu \in \mathbb{M}_{h}^{i}$,

$$
\begin{aligned}
& \frac{1}{\widehat{K}_{1}}\left(\sigma_{1, h}^{i+1}, \boldsymbol{\eta}_{1}\right)_{\Omega_{1, h}^{i}}-\left(u_{1, h}^{i+1}, \nabla \cdot \boldsymbol{\eta}_{1}\right)_{\Omega_{1, h}^{i}}-\left\langle\boldsymbol{\eta}_{1} \cdot \boldsymbol{\nu}_{h}^{i}, \lambda_{h}^{i+1}\right\rangle_{\Gamma_{h}^{i}}=-\left\langle\boldsymbol{\eta}_{1} \cdot \boldsymbol{\nu}_{h}^{i}, u_{\mathrm{D}}^{i+1}\right\rangle_{\partial \Omega_{h}}, \\
& -\left(\nabla \cdot \sigma_{1, h}^{i+1}, q_{1}\right)_{\Omega_{1, h}^{i}}-\frac{1}{\Delta t}\left(u_{1, h}^{i+1}, q_{1}\right)_{\Omega_{1, h}^{i}}+\frac{1}{\Delta t}\left({\overline{u_{1}, h}}^{i}, q_{1}\right)_{\Omega_{1, h}^{i}}=-\left(f_{1}^{i+1}, q_{1}\right)_{\Omega_{1, h}^{i}}, \\
& \frac{1}{\widehat{K}_{\mathrm{s}}}\left(\boldsymbol{\sigma}_{\mathrm{s}, h}^{i+1}, \boldsymbol{\eta}_{\mathrm{s}}\right)_{\Omega_{\mathrm{s}, h}^{i}}-\left(u_{\mathrm{s}, h}^{i+1}, \nabla \cdot \boldsymbol{\eta}_{\mathrm{s}}\right)_{\Omega_{\mathrm{s}, h}^{i}}+\left\langle\boldsymbol{\eta}_{\mathrm{s}} \cdot \nu_{h}^{i}, \lambda_{h}^{i+1}\right\rangle_{\Gamma_{h}^{i}}=0, \\
& -\left(\nabla \cdot \sigma_{\mathrm{s}, h}^{i+1}, q_{\mathrm{s}}\right)_{\Omega_{\mathrm{s}, h}^{i}}-\frac{1}{\Delta t}\left(u_{\mathrm{s}, h}^{i+1}, q_{\mathrm{s}}\right)_{\Omega_{\mathrm{s}, h}^{i}}+\frac{1}{\Delta t}\left({\overline{u_{\mathrm{s}, h}}}^{i}, q_{\mathrm{s}}\right)_{\Omega_{\mathrm{s}, h}^{i}}=-\left(f_{\mathrm{s}}^{i+1}, q_{\mathrm{s}}\right)_{\Omega_{\mathrm{s}, h}^{i}}, \\
& \left(\widehat{\beta}^{-1}\left(\nu_{h}^{i}\right) \mathbf{V}_{h}^{i+1} \cdot \nu_{h}^{i}, \mathbf{Y} \cdot \nu_{h}^{i}\right)_{\Gamma_{h}^{i}}+\Delta t \widehat{\alpha}\left(\nabla_{\Gamma} \mathbf{V}_{h}^{i+1}, \nabla_{\Gamma} \mathbf{Y}\right)_{\Gamma_{h}^{i}} \\
& +\widehat{S}\left(\mathbf{Y} \cdot \boldsymbol{\nu}_{h}^{i}, \lambda_{h}^{i+1}\right)_{\Gamma_{h}^{i}}=-\widehat{\alpha}\left(\nabla_{\Gamma} \mathbf{X}^{i}, \nabla_{\Gamma} \mathbf{Y}\right)_{\Gamma_{h}^{i}}, \\
& \widehat{S}\left(\mathbf{V}_{h}^{i+1} \cdot \nu_{h}^{i}, \mu\right)_{\Gamma_{h}^{i}}-\left\langle\sigma_{1, h}^{i+1} \cdot \nu_{h}^{i}, \mu\right\rangle_{\Gamma_{h}^{i}}+\left\langle\sigma_{\mathrm{s}, h}^{i+1} \cdot \nu_{h}^{i}, \mu\right\rangle_{\Gamma_{h}^{i}}=0,
\end{aligned}
$$

where the discrete spaces are defined over the current (known) domain $\Omega_{h}^{i}, \Gamma_{h}^{i}$. We then use the space discrete version of (4.1) to compute the new interface $\Gamma_{h}^{i+1}$, followed by the space discrete version of (4.2), (4.3) to compute the map $\Phi_{i+1, h}: \Omega_{h}^{i} \rightarrow \Omega_{h}^{i+1}$. 
REMARK 5.2 (Finite Element Space For Domain Velocity) The extension (4.2) of $\mathbf{V}_{h}^{i+1}$ to all of $\Omega_{h}^{i}$ is computed by solving a discrete Laplace equation using a finite element space $\mathbb{L}_{h}^{i}$ on $\Omega_{h}^{i}$ whose restriction to $\Gamma_{h}^{i}$ contains $\mathbb{Y}_{h}^{i}$. Because of (4.1), (4.3), the shape of the tetrahedral elements $T$ in $\Omega_{h}^{i}$ must be representable by functions in $\mathbb{L}_{h}^{i}$, i.e. the parametrization of $T$ must be expressed as a linear combination of basis functions in the local finite element space of $\mathbb{L}_{h}^{i}$. For example, this is achieved when $\mathbb{L}_{h}^{i}$ is piecewise linear and $\Omega_{h}^{i}$ consists of affine tetrahedra.

The space discrete version of the temperature update formula (4.7) is then given by

$$
{\overline{u_{j, h}}}^{i}:=\left[u_{j, h}^{i}-\Delta t \frac{1}{\widehat{K}_{j}} \Pi_{\mathbb{Q}_{j, h}^{i}}\left(\sigma_{j, h}^{i} \cdot d_{\mathbb{V}_{h}^{i}} \mathbf{V}_{h}^{i}\right)\right] \circ \Phi_{i, h}^{-1}, \text { for } j=1, \mathrm{~s},
$$

where $\Pi_{\mathbb{Q}_{j, h}^{i}}: L^{2}\left(\Omega_{j, h}^{i}\right) \rightarrow \mathbb{Q}_{j, h}^{i}$ is the standard $L^{2}$ projection onto $\mathbb{Q}_{j, h}^{i}$ and $\ell_{\mathfrak{\mathbb { V }}_{h}^{i}}: H_{0}^{1}\left(\Omega_{h}^{i}\right) \rightarrow \stackrel{\mathbb{V}}{h}_{h}^{i}$ is a suitable interpolant; see Section 5.2 for a description of these operators and Section 5.4 for the reasons we need them. Iterating this procedure gives the fully discrete approximation of (3.11), (3.12).

Just as in Section 4.3, we drop the time index notation when considering (5.1), (5.2) at a single time step. This leads to a fully discrete version of (4.14).

VARiational Formulation 5.3 Find $\left(\sigma_{1, h}, \sigma_{\mathrm{s}, h}, \mathbf{V}_{h}\right)$ in $\mathbb{Z}_{h}$ and $\left(u_{1, h}, u_{\mathrm{s}, h}, \lambda_{h}\right)$ in $\mathbb{T}_{h}$ such that

$$
\begin{aligned}
& a_{h}\left(\left(\boldsymbol{\eta}_{1}, \boldsymbol{\eta}_{\mathrm{s}}, \mathbf{Y}\right),\left(\sigma_{1, h}, \boldsymbol{\sigma}_{\mathrm{s}, h}, \mathbf{V}_{h}\right)\right)+b_{h}\left(\left(\boldsymbol{\eta}_{\mathrm{l}}, \boldsymbol{\eta}_{\mathrm{s}}, \mathbf{Y}\right),\left(u_{\mathrm{l}, h}, u_{\mathrm{s}, h}, \lambda_{h}\right)\right)=\chi_{h}\left(\boldsymbol{\eta}_{\mathrm{l}}, \boldsymbol{\eta}_{\mathrm{s}}, \mathbf{Y}\right), \\
& +b_{h}\left(\left(\boldsymbol{\sigma}_{\mathrm{l}, h}, \boldsymbol{\sigma}_{\mathrm{s}, h}, \mathbf{V}_{h}\right),\left(q_{\mathrm{l}}, q_{\mathrm{s}}, \mu\right)\right)-c_{h}\left(\left(q_{\mathrm{l}}, q_{\mathrm{s}}, \mu\right),\left(u_{1, h}, u_{\mathrm{s}, h}, \lambda_{h}\right)\right)=\psi_{h}\left(q_{1}, q_{\mathrm{s}}, \mu\right),
\end{aligned}
$$

for all $\left(\boldsymbol{\eta}_{\mathrm{l}}, \boldsymbol{\eta}_{\mathrm{s}}, \mathbf{Y}\right)$ in $\mathbb{Z}_{h}$, and $\left(q_{1}, q_{\mathrm{s}}, \mu\right)$ in $\mathbb{T}_{h}$.

The discrete version of the forms in Section 4.3.1 are defined in the obvious way. The discrete product spaces are defined similar to (4.12), (4.13): $\mathbb{Z}_{h}=\mathbb{V}_{1, h}(0) \times \mathbb{V}_{\mathrm{s}, h} \times \mathbb{Y}_{h}, \mathbb{T}_{h}=\mathbb{Q}_{1, h} \times \mathbb{Q}_{\mathrm{s}, h} \times \mathbb{M}_{h}$.

5.1.3 Discrete norms. The discrete multiplier norm is slightly different. We first introduce a discrete version of the $H^{1 / 2}\left(\Gamma_{h}\right)$ norm. For any $\mu \in H^{1 / 2}\left(\Gamma_{h}\right)$, define the discrete version of (3.7):

$$
\|\mu\|_{H_{1, h}^{1 / 2}\left(\Gamma_{h}\right)}:=\sup _{\eta_{1} \in \mathbb{V}_{1, h}(0)} \frac{\left\langle\boldsymbol{\eta}_{1} \cdot \boldsymbol{\nu}_{h}, \mu\right\rangle_{\Gamma_{h}}}{\left\|\boldsymbol{\eta}_{1}\right\|_{H\left(\operatorname{div}, \Omega_{1, h}\right)}}, \quad\|\mu\|_{H_{\mathrm{s}, h}^{1 / 2}\left(\Gamma_{h}\right)}:=\sup _{\boldsymbol{\eta}_{\mathrm{s}} \in \mathbb{V}_{\mathrm{s}, h}} \frac{\left\langle\boldsymbol{\eta}_{\mathrm{s}} \cdot \boldsymbol{\nu}_{h}, \mu\right\rangle_{\Gamma_{h}}}{\left\|\boldsymbol{\eta}_{\mathrm{s}}\right\|_{H\left(\operatorname{div}, \Omega_{\mathrm{s}, h}\right)}} .
$$

Clearly, for $j=1, \mathrm{~s},\|\mu\|_{H_{j, h}^{1 / 2}\left(\Gamma_{h}\right)} \leqslant\|\mu\|_{H_{j}^{1 / 2}\left(\Gamma_{h}\right)}$ and $\left\langle\boldsymbol{\eta} \cdot \boldsymbol{\nu}_{h}, \mu\right\rangle_{\Gamma_{h}} \leqslant\|\boldsymbol{\eta}\|_{H\left(\operatorname{div}, \Omega_{j, h}\right)}\|\mu\|_{H_{j, h}^{1 / 2}\left(\Gamma_{h}\right)}$ (discrete Schwarz inequality). We shall also use a discrete version of the $H^{-1}\left(\Gamma_{h}\right)$ norm to control the mean value of $\mu \in \mathbb{M}_{h}$. For all $\mathbf{v}$ in $H^{-1}\left(\Gamma_{h}\right)$, define

$$
\|\mathbf{v}\|_{H_{h}^{-1}\left(\Gamma_{h}\right)}:=\sup _{\mathbf{Y} \in \mathbb{Y}_{h}} \frac{\langle\mathbf{v}, \mathbf{Y}\rangle_{\Gamma_{h}}}{\|\mathbf{Y}\|_{H^{1}\left(\Gamma_{h}\right)}}
$$

which also satisfies $\|\mathbf{v}\|_{H_{h}^{-1}\left(\Gamma_{h}\right)} \leqslant\|\mathbf{v}\|_{H^{-1}\left(\Gamma_{h}\right)}$ and $\langle\mathbf{v}, \mathbf{Y}\rangle_{\Gamma_{h}} \leqslant\|\mathbf{v}\|_{H_{h}^{-1}\left(\Gamma_{h}\right)}\|\mathbf{Y}\|_{H^{1}\left(\Gamma_{h}\right)}$ (discrete Schwarz inequality). Then the discrete version of $\left\|\left(q_{1}, q_{\mathrm{s}}, \mu\right)\right\|_{\mathbb{T}^{\diamond}}^{2}$ is $\left\|\left(q_{1}, q_{\mathrm{s}}, \mu\right)\right\|_{\mathbb{T}_{h}^{\circ}}^{2}=\left\|q_{1}\right\|_{L^{2}\left(\Omega_{1, h}\right)}^{2}+$ $\left\|q_{\mathrm{s}}\right\|_{L^{2}\left(\Omega_{\mathrm{s}, h}\right)}^{2}+\|\mu\|_{H_{h}^{1 / 2}\left(\Gamma_{h}\right)}^{2}$, where

$$
\|\mu\|_{H_{h}^{1 / 2}\left(\Gamma_{h}\right)}:=\frac{1}{2}\left(\|\mu\|_{H_{1, h}^{1 / 2}\left(\Gamma_{h}\right)}+\|\mu\|_{H_{\mathrm{s}, h}^{1 / 2}\left(\Gamma_{h}\right)}\right) .
$$


and the discrete version of (4.16) is

$$
\begin{aligned}
\left\|\left(q_{1}, q_{\mathrm{s}}, \mu\right)\right\|_{\mathbb{T}_{h}}^{2}= & \left\|\tilde{q}_{1}\right\|_{L^{2}\left(\Omega_{1, h}\right)}^{2}+\left\|\tilde{q}_{\mathrm{s}}\right\|_{L^{2}\left(\Omega_{\mathrm{s}, h}\right)}^{2} \\
& +\left\|\mu-\hat{q}_{1}\right\|_{H_{1, h}^{1 / 2}\left(\Gamma_{h}\right)}^{2}+\left\|\mu-\hat{q}_{\mathrm{s}}\right\|_{H_{\mathrm{s}, h}^{1 / 2}\left(\Gamma_{h}\right)}^{2}+\widehat{S}\left\|\mu \nu_{h}\right\|_{H_{h}^{-1}\left(\Gamma_{h}\right)}^{2} .
\end{aligned}
$$

A discrete version of Proposition 4.7 also holds, i.e. $\left\|\left(q_{1}, q_{\mathrm{s}}, \mu\right)\right\|_{\mathbb{T}_{h}^{\diamond}} \approx\left\|\left(q_{1}, q_{\mathrm{s}}, \mu\right)\right\|_{\mathbb{T}_{h}}$.

The discrete version of the primal norm (4.15) is also slightly different. It requires a discrete version of the $H^{-1 / 2}\left(\Gamma_{h}\right)$ norm to control the mean value of $\mathbf{Y} \cdot \boldsymbol{\nu}_{h}$ for $\mathbf{Y} \in \mathbb{Y}_{h}$. For any $\mathbf{Y} \cdot \boldsymbol{\nu}_{h} \in$ $H^{-1 / 2}\left(\Gamma_{h}\right)$, define

$$
\left\|\mathbf{Y} \cdot \nu_{h}\right\|_{H_{h}^{-1 / 2}\left(\Gamma_{h}\right)}:=\sup _{\mu_{h} \in \mathbb{M}_{h}} \frac{\left\langle\mathbf{Y} \cdot \nu_{h}, \mu_{h}\right\rangle_{\Gamma_{h}}}{\left\|\mu_{h}\right\|_{H_{h}^{1 / 2}\left(\Gamma_{h}\right)}},
$$

Clearly, $\left\langle\mathbf{Y} \cdot \nu_{h}, \mu_{h}\right\rangle_{\Gamma_{h}} \leqslant\left\|\mathbf{Y} \cdot \nu_{h}\right\|_{H_{h}^{-1 / 2}\left(\Gamma_{h}\right)}\left\|\mu_{h}\right\|_{H_{h}^{1 / 2}\left(\Gamma_{h}\right)}$ (discrete Schwarz inequality). Then the discrete version of $\left\|\left(\boldsymbol{\eta}_{\mathbf{l}}, \boldsymbol{\eta}_{\mathrm{s}}, \mathbf{Y}\right)\right\|_{\mathbb{Z}}^{2}$ is obtained by replacing $\|\mathbf{Y} \cdot \boldsymbol{\nu}\|_{H^{-1 / 2}(\Gamma)}$ with $\left\|\mathbf{Y} \cdot \boldsymbol{\nu}_{h}\right\|_{H_{h}^{-1 / 2}\left(\Gamma_{h}\right)}$. A discrete version of Proposition 4.6 also holds.

\subsection{Space assumptions}

To prove well-posedness of the discrete system, we must prove the discrete version of Lemmas 4.8, 4.9, and 4.10. In addition, we want to obtain discrete versions of Theorems 4.14 and 4.16. To facilitate this, we make the following general assumptions on the choice of finite dimensional subspaces (see Section 5.5 for the specific spaces used).

Let $\mathbb{V}_{h}$ be a conforming finite dimensional subspace, i.e. $\mathbb{V}_{h} \subset \mathbb{V} \equiv H(\operatorname{div}, \Omega)$, and define

$$
\mathbb{V}_{h}:=\left\{\boldsymbol{\eta} \in \mathbb{V}_{h}: \boldsymbol{\eta} \cdot \boldsymbol{\nu}_{h}=0 \text {, on } \partial \Omega_{h}\right\} \subset\left\{\boldsymbol{\eta} \in \mathbb{V}:\left\langle\boldsymbol{\eta} \cdot \boldsymbol{\nu}_{h}, q\right\rangle_{\partial \Omega_{h}}=0, \forall q \in H^{1}\left(\Omega_{h}\right)\right\}
$$

Furthermore, assume that for any $\boldsymbol{\eta}$ in $\mathbb{\mathbb { V }}_{h}$, we have $\left.\boldsymbol{\eta}\right|_{\Omega_{1, h}} \in \mathbb{V}_{1, h}(0)$ and $\left.\boldsymbol{\eta}\right|_{\Omega_{\mathrm{s}, h}} \in \mathbb{V}_{\mathrm{s}, h}$.

Next, take $\stackrel{\mathbb{V}}{1, h}_{1, h}=\left\{\boldsymbol{\eta}_{1} \in \mathbb{V}_{1, h}: \boldsymbol{\eta}_{1} \cdot \boldsymbol{\nu}_{h}=0\right.$, on $\left.\partial \Omega_{1, h}\right\}$ and $\hat{\mathbb{Q}}_{1, h}=\left\{q \in \mathbb{Q}_{1, h}: \int_{\Omega_{1, h}} q d x=0\right\}$, and assume that $\nabla \cdot \mathbb{V}_{1, h}=\mathbb{Q}_{1, h}, \nabla \cdot \stackrel{\bullet}{1}_{1, h}=\hat{\mathbb{Q}}_{1, h}$, and $\mathbb{V}_{1, h}$ contains continuous piecewise linear functions on $\Gamma_{h}$. Analogous definitions are made for $\mathbb{V}_{\mathrm{s}, h}$ and $\mathbb{Q}_{\mathrm{s}, h}$. Moreover, assume $\left(\mathbb{V}_{1, h}, \mathbb{Q}_{1, h}\right)$ and $\left(\mathbb{V}_{\mathrm{s}, h}, \mathbb{Q}_{\mathrm{s}, h}\right)$ satisfy

$$
\sup _{\boldsymbol{\eta}_{1} \in \mathbb{V}_{1, h}} \frac{-\left(\nabla \cdot \boldsymbol{\eta}_{1}, q_{1}\right)_{\Omega_{1, h}}}{\left\|\boldsymbol{\eta}_{1}\right\|_{\left.H\left(\operatorname{div}, \Omega_{1, h}\right)\right)}} \geqslant c\left\|q_{1}\right\|_{L^{2}\left(\Omega_{1, h}\right)}, \quad \sup _{\boldsymbol{\eta}_{\mathrm{s}} \in \mathbb{V}_{\mathrm{s}, h}} \frac{-\left(\nabla \cdot \boldsymbol{\eta}_{\mathrm{s}}, q_{\mathrm{s}}\right)_{\Omega_{\mathrm{s}, h}}}{\left\|\boldsymbol{\eta}_{\mathrm{s}}\right\|_{\left.H\left(\operatorname{div}, \Omega_{\mathrm{s}, h}\right)\right)}} \geqslant c\left\|q_{\mathrm{s}}\right\|_{L^{2}\left(\Omega_{\mathrm{s}, h}\right)},
$$

for all $q_{1} \in \mathbb{Q}_{1, h}, q_{\mathrm{s}} \in \mathbb{Q}_{\mathrm{s}, h}$, with $c$ independent of $h$ and that an analogous condition is satisfied for $\left(\mathbb{V}_{1, h}, \hat{\mathbb{Q}}_{1, h}\right)$ and $\left(\mathbb{V}_{\mathrm{s}, h}, \hat{\mathbb{Q}}_{\mathrm{s}, h}\right)$. This implies that we can solve the discrete mixed form of Laplace's equation. As for $\mathbb{Y}_{h}$ and $\mathbb{M}_{h}$, assume they are spaces of continuous functions.

Regarding (5.3), we have a "Fortin interpolant" $[9,11]{d_{\mathbb{V}_{h}}}: H_{0}^{1}\left(\Omega_{h}\right) \rightarrow \stackrel{\circ}{V}_{h}$ that satisfies for any $\mathbf{V} \in H_{0}^{1}\left(\Omega_{h}\right)$ :

$$
\left\|{\mathcal{Q}_{\mathbb{V}_{h}}} \mathbf{V}\right\|_{L^{2}\left(\Omega_{h}\right)} \leqslant C\|\mathbf{V}\|_{H^{1}\left(\Omega_{h}\right)} \text {, and }\left(q, \nabla \cdot \mathbf{V}-\nabla \cdot{d_{\mathbb{V}_{h}}} \mathbf{V}\right)_{\Omega_{j, h}}=0, \forall q \in \mathbb{Q}_{j, h} \text {, for } j=1, \mathrm{~s} .
$$

And the $L^{2}$ projections $\Pi_{\mathbb{Q}_{j, h}}: L^{2}\left(\Omega_{j, h}\right) \rightarrow \mathbb{Q}_{j, h}$ (for $j=1$, s) satisfy for any $v \in L^{2}\left(\Omega_{j, h}\right)$ :

$$
\left\|\Pi_{\mathbb{Q}_{j, h}} v\right\|_{L^{2}\left(\Omega_{j, h}\right)} \leqslant\|v\|_{L^{2}\left(\Omega_{j, h}\right)} \text {, and }\left(q, v-\Pi_{\mathbb{Q}_{j, h}} v\right)_{\Omega_{j, h}}=0, \forall q \in \mathbb{Q}_{j, h} \text {, for } j=1, \mathrm{~s} \text {. }
$$




\subsection{Well-posedness}

We follow a similar outline as Section 4.5.

\subsubsection{Main conditions.}

LEMMA 5.4 (Continuity of Forms)

$$
\begin{aligned}
& \left|a_{h}\left(\left(\boldsymbol{\eta}_{1}, \boldsymbol{\eta}_{\mathrm{s}}, \mathbf{Y}\right),\left(\boldsymbol{\sigma}_{1}, \boldsymbol{\sigma}_{\mathrm{s}}, \mathbf{V}\right)\right)\right| \leqslant C_{a_{h}}\left\|\left(\boldsymbol{\eta}_{1}, \boldsymbol{\eta}_{\mathrm{s}}, \mathbf{Y}\right)\right\|_{\mathbb{Z}_{h}}\left\|\left(\sigma_{1}, \boldsymbol{\sigma}_{\mathrm{s}}, \mathbf{V}\right)\right\|_{\mathbb{Z}_{h}}, \forall\left(\boldsymbol{\eta}_{\mathrm{l}}, \boldsymbol{\eta}_{\mathrm{s}}, \mathbf{Y}\right),\left(\sigma_{1}, \boldsymbol{\sigma}_{\mathrm{s}}, \mathbf{V}\right) \in \mathbb{Z}_{h}, \\
& \left|b_{h}\left(\left(\boldsymbol{\eta}_{1}, \boldsymbol{\eta}_{\mathrm{s}}, \mathbf{Y}\right),\left(q_{1}, q_{\mathrm{s}}, \mu\right)\right)\right| \leqslant C_{b_{h}}\left\|\left(\boldsymbol{\eta}_{1}, \boldsymbol{\eta}_{\mathrm{s}}, \mathbf{Y}\right)\right\|_{\mathbb{Z}_{h}}\left\|\left(q_{1}, q_{\mathrm{s}}, \mu\right)\right\|_{\mathbb{T}_{h}}, \\
& \forall\left(\boldsymbol{\eta}_{1}, \boldsymbol{\eta}_{\mathrm{s}}, \mathbf{Y}\right) \in \mathbb{Z}_{h},\left(q_{1}, q_{\mathrm{s}}, \mu\right) \in \mathbb{T}_{h}, \\
& \left|c_{h}\left(\left(q_{1}, q_{\mathrm{s}}, \mu\right),\left(u_{1}, u_{\mathrm{s}}, \lambda\right)\right)\right| \leqslant \Delta t^{-1}\left(\left\|q_{1}\right\|_{L^{2}\left(\Omega_{1, h}\right)}\left\|u_{1}\right\|_{L^{2}\left(\Omega_{1, h}\right)}+\left\|q_{\mathrm{s}}\right\|_{L^{2}\left(\Omega_{\mathrm{s}, h}\right)}\left\|u_{\mathrm{s}}\right\|_{L^{2}\left(\Omega_{\mathrm{s}, h}\right)}\right), \\
& \forall\left(q_{1}, q_{\mathrm{s}}, \mu\right),\left(u_{1}, u_{\mathrm{s}}, \lambda\right) \in \mathbb{T}_{h}, \\
& \left|\chi_{h}\left(\boldsymbol{\eta}_{\mathrm{l}}, \boldsymbol{\eta}_{\mathrm{s}}, \mathbf{Y}\right)\right| \leqslant C_{\chi_{h}}\left\|\left(\boldsymbol{\eta}_{\mathrm{l}}, \boldsymbol{\eta}_{\mathrm{s}}, \mathbf{Y}\right)\right\|_{\mathbb{Z}_{h}}, \forall\left(\boldsymbol{\eta}_{\mathrm{l}}, \boldsymbol{\eta}_{\mathrm{s}}, \mathbf{Y}\right) \in \mathbb{Z}_{h}, \\
& \left|\psi_{h}\left(q_{1}, q_{\mathrm{s}}, \mu\right)\right| \leqslant C_{\psi_{h}}\left\|\left(q_{1}, q_{\mathrm{s}}, \mu\right)\right\|_{\mathbb{T}_{h}}, \forall\left(q_{1}, q_{\mathrm{s}}, \mu\right) \in \mathbb{T}_{h},
\end{aligned}
$$

where $C_{a_{h}}, C_{b_{h}}, C_{\chi_{h}}, C_{\psi_{h}}>0$ are constants that depend on physical parameters and domain geometry. In addition, $C_{\chi_{h}}$ depends on $u_{\mathrm{D}}, \Delta t^{-1 / 2}$, and $C_{\psi_{h}}$ depends on $f_{1}, f_{\mathrm{s}}, \overline{u_{1}}, \overline{u_{\mathrm{s}}}$ and $\Delta t^{-1}$.

Proof. The proof is analogous to the proof of Lemma 4.8. Minor modifications are: one must use the discrete Schwarz inequalities associated with the discrete $H_{1, h}^{1 / 2}, H_{\mathrm{s}, h}^{1 / 2}$, and $H_{h}^{-1 / 2}$ norms, and use the discrete versions of Propositions 4.6 and 4.7.

LEMMA 5.5 (Coercivity) Let $\left(\boldsymbol{\eta}_{\mathrm{l}}, \boldsymbol{\eta}_{\mathrm{s}}, \mathbf{Y}\right) \in \mathbb{Z}_{h}$ with $b_{h}\left(\left(\boldsymbol{\eta}_{1}, \boldsymbol{\eta}_{\mathrm{s}}, \mathbf{Y}\right),\left(q_{1}, q_{\mathrm{s}}, \mu\right)\right)=0$ for all $\left(q_{1}, q_{\mathrm{s}}, \mu\right) \in \mathbb{T}_{h}$. Then,

$$
\left|a_{h}\left(\left(\boldsymbol{\eta}_{1}, \boldsymbol{\eta}_{\mathrm{s}}, \mathbf{Y}\right),\left(\boldsymbol{\eta}_{\mathbf{l}}, \boldsymbol{\eta}_{\mathrm{s}}, \mathbf{Y}\right)\right)\right| \geqslant C\left\|\left(\boldsymbol{\eta}_{\mathbf{l}}, \boldsymbol{\eta}_{\mathrm{s}}, \mathbf{Y}\right)\right\|_{\mathbb{Z}_{h}}^{2}
$$

where $C>0$ is a constant depending on $\widehat{S}, \widehat{K}, \widehat{K_{\mathrm{s}}}$, and the domain. This is true even if $\widehat{\beta} \rightarrow \infty$.

Proof. Follows the same argument as in Lemma 4.9, except the discrete $H_{h}^{-1 / 2}$ norm (5.9) is used.

LEMMA 5.6 (Inf-Sup) For all $\left(q_{1}, q_{\mathrm{s}}, \mu\right) \in \mathbb{T}_{h}$, the following "inf-sup" condition holds

$$
\sup _{\left(\boldsymbol{\eta}_{\mathrm{l}}, \boldsymbol{\eta}_{\mathrm{s}}, \mathbf{Y}\right) \in \mathbb{Z}_{h}} \frac{b_{h}\left(\left(\boldsymbol{\eta}_{\mathrm{l}}, \boldsymbol{\eta}_{\mathrm{s}}, \mathbf{Y}\right),\left(q_{1}, q_{\mathrm{s}}, \mu\right)\right)}{\left\|\left(\boldsymbol{\eta}_{\mathrm{l}}, \boldsymbol{\eta}_{\mathrm{s}}, \mathbf{Y}\right)\right\|_{\mathbb{Z}_{h}^{\diamond}}} \geqslant C\left\|\left(q_{1}, q_{\mathrm{s}}, \mu\right)\right\|_{\mathbb{T}_{h}},
$$

where $C>0$ depends on the domain and $\widehat{S}$. If $\left\|\left(\boldsymbol{\eta}_{1}, \boldsymbol{\eta}_{\mathrm{s}}, \mathbf{Y}\right)\right\|_{\mathbb{Z}_{h}^{\diamond}}$ is replaced by $\left\|\left(\boldsymbol{\eta}_{\mathbf{l}}, \boldsymbol{\eta}_{\mathrm{s}}, \mathbf{Y}\right)\right\|_{\mathbb{Z}_{h}}$ in the denominator, then the inf-sup still holds, except $C$ also depends on $\widehat{K}_{\mathrm{l}}, \widehat{K}_{\mathrm{s}}, \widehat{\alpha}$, and $\widehat{\beta}_{-}$. Furthermore, $C$ does not depend on the time step $\Delta t$, as long as $\Delta t \leqslant 1$.

Proof. Starting as we did in the proof of Lemma 4.10, we have

$$
\begin{aligned}
b_{h}\left(\left(\boldsymbol{\eta}_{\mathrm{l}}, \boldsymbol{\eta}_{\mathrm{s}}, \mathbf{Y}\right),\left(q_{1}, q_{\mathrm{s}}, \mu\right)\right)=-\left(\nabla \cdot \boldsymbol{\eta}_{\mathrm{l}}, \tilde{q}_{1}\right)_{\Omega_{1, h}}-\left(\nabla \cdot \boldsymbol{\eta}_{\mathrm{s}}, \tilde{q}_{\mathrm{s}}\right)_{\Omega_{\mathrm{s}, h}}-\left\langle\boldsymbol{\eta}_{\mathrm{l}} \cdot \boldsymbol{\nu}_{h}, \mu-\hat{q}_{1}\right\rangle_{\Gamma_{h}} \\
+\left\langle\boldsymbol{\eta}_{\mathrm{s}} \cdot \boldsymbol{\nu}_{h}, \mu-\hat{q}_{\mathrm{s}}\right\rangle_{\Gamma_{h}}+\widehat{S}\left(\mathbf{Y} \cdot \boldsymbol{\nu}_{h}, \mu\right)_{\Gamma_{h}} .
\end{aligned}
$$


Next, let us focus on $-\left(\nabla \cdot \boldsymbol{\eta}_{\mathrm{s}}, \tilde{q}_{\mathrm{s}}\right)_{\Omega_{\mathrm{s}, h}}+\left\langle\boldsymbol{\eta}_{\mathrm{s}} \cdot \boldsymbol{\nu}_{h}, \mu-\hat{q}_{\mathrm{s}}\right\rangle_{\Gamma_{h}}$ only. By (5.10), there exists a unique $(\mathbf{w}, \omega)$ in $\left(\stackrel{\ominus}{\mathrm{V}}_{\mathrm{s}, h}, \hat{\mathbb{Q}}_{\mathrm{s}, h}\right)$ such that

$$
\begin{array}{rlrl}
(\mathbf{w}, \mathbf{v})_{\Omega_{\mathrm{s}, h}}-(\omega, \nabla \cdot \mathbf{v})_{\Omega_{\mathrm{s}, h}} & =0, & & \forall \mathbf{v} \in \grave{\mathbb{V}}_{\mathrm{s}, h}, \\
-(\nabla \cdot \mathbf{w}, r) \Omega_{\mathrm{s}, h} & =\left(\tilde{q}_{\mathrm{s}}, r\right)_{\Omega_{\mathrm{s}, h},}, & \forall r \in \hat{\mathbb{Q}}_{\mathrm{s}, h},
\end{array}
$$

and $\|\mathbf{w}\|_{H\left(\operatorname{div}, \Omega_{\mathrm{s}, h}\right)} \leqslant C_{0}\left\|\tilde{q}_{\mathrm{s}}\right\|_{L^{2}\left(\Omega_{\mathrm{s}, h}\right)}$. By (5.5), there exists $\boldsymbol{\xi} \in \mathbb{V}_{\mathrm{s}, h}$ such that

$$
\left\langle\boldsymbol{\xi} \cdot \nu_{h}, \mu-\hat{q}_{\mathrm{s}}\right\rangle_{\Gamma_{h}}=\left\|\mu-\hat{q}_{\mathrm{s}}\right\|_{H_{\mathrm{s}, h}^{1 / 2}\left(\Gamma_{h}\right)}^{2}, \quad\|\boldsymbol{\xi}\|_{H\left(\mathrm{div}, \Omega_{\mathrm{s}, h}\right)}=\left\|\mu-\hat{q}_{\mathrm{s}}\right\|_{H_{\mathrm{s}, h}^{1 / 2}\left(\Gamma_{h}\right)} .
$$

Similar to (5.13), there exists a $\mathbf{z}$ in $\stackrel{\bigotimes}{\mathrm{V}}, h_{\mathrm{s}}$ such that

$$
-\nabla \cdot \mathbf{z}=\nabla \cdot \boldsymbol{\xi}-\frac{1}{\left|\Omega_{\mathrm{s}, h}\right|}\left(\int_{\Gamma_{h}} \boldsymbol{\xi} \cdot \boldsymbol{\nu}_{h}\right), \text { on } \Omega_{\mathrm{s}, h}, \quad\|\mathbf{z}\|_{H\left(\mathrm{div}, \Omega_{\mathrm{s}, h}\right)} \leqslant C_{1}\|\boldsymbol{\xi}\|_{H\left(\mathrm{div}, \Omega_{\mathrm{s}, h}\right)} .
$$

Now let $\mathbf{d}=\mathbf{z}+\boldsymbol{\xi}$. Then,

$$
\nabla \cdot \mathbf{d}=\frac{1}{\left|\Omega_{\mathrm{s}, h}\right|}\left(\int_{\Gamma_{h}} \boldsymbol{\xi} \cdot \nu_{h}\right), \text { on } \Omega_{\mathrm{s}, h}, \quad \mathbf{d} \cdot \nu_{h}=\boldsymbol{\xi} \cdot \nu_{h}, \text { on } \Gamma_{h},
$$

where $\|\mathbf{d}\|_{H\left(\mathrm{div}, \Omega_{\mathrm{s}, h}\right)} \leqslant\left(1+C_{1}\right)\|\boldsymbol{\xi}\|_{H\left(\mathrm{div}, \Omega_{\mathrm{s}, h}\right)}=\left(1+C_{1}\right)\left\|\mu-\hat{q}_{\mathrm{s}}\right\|_{H_{\mathrm{s}, h}^{1 / 2}\left(\Gamma_{h}\right)}$.

Next, define $\mathbf{y}:=\mathbf{w}+\mathbf{d} \in \mathbb{V}_{\mathrm{s}, h}$ and note $\|\mathbf{y}\|_{H\left(\operatorname{div}, \Omega_{\mathrm{s}, h}\right)} \leqslant C_{0}\left\|\tilde{q}_{\mathrm{s}}\right\|_{L^{2}\left(\Omega_{\mathrm{s}, h}\right)}+\left(1+C_{1}\right) \| \mu-$ $\hat{q}_{\mathrm{s}} \|_{H_{\mathrm{s}, h}^{1 / 2}\left(\Gamma_{h}\right)}$. Thus, setting $\boldsymbol{\eta}_{\mathrm{s}}:=\mathbf{y} /\|\mathbf{y}\|_{H\left(\mathrm{div}, \Omega_{\mathrm{s}, h}\right)}$ gives

$$
\begin{aligned}
-\left(\nabla \cdot \boldsymbol{\eta}_{\mathrm{s}}, \tilde{q}_{\mathrm{s}}\right)_{\Omega_{\mathrm{s}, h}}+\left\langle\boldsymbol{\eta}_{\mathrm{s}} \cdot \boldsymbol{\nu}_{h}, \mu-\hat{q}_{\mathrm{s}}\right\rangle_{\Gamma_{h}} & =\frac{1}{\|\mathbf{y}\|_{H\left(\operatorname{div}, \Omega_{\mathrm{s}, h}\right)}}\left(\left\|\tilde{q}_{\mathrm{s}}\right\|_{L^{2}\left(\Omega_{\mathrm{s}, h}\right)}^{2}+\left\langle\mathbf{d} \cdot \boldsymbol{\nu}_{h}, \mu-\hat{q}_{\mathrm{s}}\right\rangle_{\Gamma_{h}}\right) \\
& \geqslant C_{2}\left(\left\|\tilde{q}_{\mathrm{s}}\right\|_{L^{2}\left(\Omega_{\mathrm{s}, h}\right)}+\left\|\mu-\hat{q}_{\mathrm{s}}\right\|_{H_{\mathrm{s}, h}^{1 / 2}\left(\Gamma_{h}\right)}\right)
\end{aligned}
$$

with $\left\|\boldsymbol{\eta}_{\mathrm{s}}\right\|_{H\left(\mathrm{div}, \Omega_{\mathrm{s}, h}\right)}=1$. Similarly, there exists $\boldsymbol{\eta}_{1} \in \mathbb{V}_{1, h}(0)$ such that

$$
-\left(\nabla \cdot \boldsymbol{\eta}_{1}, \tilde{q}_{1}\right)_{\Omega_{1, h}}-\left\langle\boldsymbol{\eta}_{1} \cdot \nu_{h}, \mu-\hat{q}_{1}\right\rangle_{\Gamma_{h}} \geqslant C_{3}\left(\left\|\tilde{q}_{1}\right\|_{L^{2}\left(\Omega_{1, h}\right)}+\left\|\mu-\hat{q}_{1}\right\|_{H_{1, h}^{1 / 2}\left(\Gamma_{h}\right)}\right),
$$

with $\left\|\boldsymbol{\eta}_{1}\right\|_{H\left(\mathrm{div}, \Omega_{1, h}\right)}=1$.

By the definition of the discrete $H^{-1}\left(\Gamma_{h}\right)$ norm (5.6), there exists a $\mathbf{Y}$ in $\mathbb{Y}_{h}$ such that

$$
\left(\mathbf{Y} \cdot \nu_{h}, \mu\right)_{\Gamma_{h}}=\left\langle\mathbf{Y}, \mu \nu_{h}\right\rangle_{\Gamma_{h}}=\left\|\mu \nu_{h}\right\|_{H_{h}^{-1}\left(\Gamma_{h}\right)}, \quad\|\mathbf{Y}\|_{H^{1}\left(\Gamma_{h}\right)}=1 .
$$

Combining the above results gives the assertion.

5.3.2 Summary. A discussion analogous to the one in Section 4.5.2 applies to the fully discrete problem also. Hence, the discrete problem is well-posed, but one must modify the norm $\|\cdot\|_{\mathbb{Z}_{h}}^{2}$ to include an extra factor of $\Delta t$ multiplying $\left\|\nabla \cdot \eta_{1}\right\|_{L^{2}\left(\Omega_{1, h}\right)}^{2},\left\|\nabla \cdot \eta_{\mathrm{s}}\right\|_{L^{2}\left(\Omega_{\mathrm{s}, h}\right)}^{2}$, and $\left\|\mathbf{Y} \cdot \boldsymbol{\nu}_{h}\right\|_{H_{h}^{-1 / 2}\left(\Gamma_{h}\right)}^{2}$. 


\subsection{Discrete estimates}

Applying the same arguments in Section 4.6.1 to the fully discrete problem (5.1), (5.2), (5.3), and using the stability properties in (5.11), (5.12), we get the fully discrete version of Theorem 4.14.

For the conservation law, the argument in Section 4.6.2 changes slightly. Recalling (5.3), the discrete counterpart of (4.30) is

$$
\int_{\Omega_{1, h}^{i}} \overline{u_{1, h}}=\int_{\Omega_{1, h}^{i-1}} u_{1, h}^{i}+\Delta t \underbrace{\int_{\Omega_{1, h}^{i-1}}\left(u_{1, h}^{i} \nabla \cdot \mathbf{V}_{h}^{i}-\widehat{K}_{1}^{-1} \Pi_{\mathbb{Q}_{1, h}^{i}}\left(\sigma_{1, h}^{i} \cdot d_{\mathbb{V}_{h}^{i}}^{i} \mathbf{V}_{h}^{i}\right)\right)}_{=: R_{1}}+O\left(\Delta t^{2}\right) .
$$

Using the properties of ${\ell_{\mathbb{V}}^{i}}_{h}$ and $\Pi_{\mathbb{Q}_{1, h}^{i}}(5.11),(5.12)$, we see that

$$
R_{1}=\int_{\Omega_{1, h}^{i-1}} u_{1, h}^{i} \nabla \cdot{\iota_{\mathbb{V}_{h}^{i}}}_{\mathbf{V}_{h}^{i}}^{i}-\int_{\Omega_{1, h}^{i-1}} \widehat{K}_{1}^{-1} \sigma_{1, h}^{i} \cdot{\ell_{\mathbb{V}_{h}^{i}}}_{\mathbf{V}_{h}^{i}}^{i}=-\int_{\Gamma_{h}^{i-1}} \lambda_{h}^{i}\left(d_{\mathbb{V}_{h}^{i}} \mathbf{V}_{h}^{i}\right) \cdot \nu_{h}^{i-1},
$$

where the last equality follows by choosing $\boldsymbol{\eta}_{1}=\left.{d_{\mathbb{V}_{h}^{i}}^{i}}_{\mathbf{V}_{h}^{i}}^{i}\right|_{\Omega_{1, h}} \in \mathbb{V}_{1, h}^{i-1}(0)$ in (5.1) on $\Omega_{1, h}^{i-1}$ for any $i \geqslant 1$. Thus, we arrive at

$$
\int_{\Omega_{1, h}^{i}}{\overline{u_{1, h}}}^{i}=\int_{\Omega_{1, h}^{i-1}} u_{1, h}^{i}-\Delta t \int_{\Gamma_{h}^{i-1}} \lambda_{h}^{i}\left(\ell_{\mathbb{V}_{h}^{i}} \mathbf{V}_{h}^{i}\right) \cdot \nu_{h}^{i-1}+O\left(\Delta t^{2}\right), \quad \text { for all } i \geqslant 1 .
$$

A similar relation holds for $u_{\mathrm{s}, h}^{i}$, except with $+\Delta t$. The rest of the derivation in Section 4.6.2 remains the same (note that $\ell_{\mathbb{V}_{h} i} \mathbf{V}_{h}^{i}$ is continuous across $\Gamma_{h}^{i-1}$ ), which delivers the fully discrete version of Theorem 4.16.

We summarize these results in the following theorem.

Theorem 5.7 (A Priori Bound and Conservation Law) Suppose (5.1), (5.2), (5.3) is solved on $\Omega_{h}^{i}$ at time index $i$, with $u_{\mathrm{D}}^{i+1}=0$, and that $\Phi_{i+1, h}$ is a bijective map with bounded inverse. Suppose this holds for $i=0, \ldots, N-1$. If $\Delta t \leqslant 1$ also satisfies

$$
\Delta t \leqslant \frac{B_{0}}{\max _{0 \leqslant i \leqslant N}\left\|\mathbf{V}_{h}^{i}\right\|_{W^{1, \infty}(\Omega)}} \quad \text { and } \quad \Delta t \leqslant B_{0} \frac{\max \left(\widehat{K}_{1}, \widehat{K_{\mathrm{s}}}\right)}{\max _{0 \leqslant i \leqslant N}\left\|\mathbf{V}_{h}^{i}\right\|_{W^{1, \infty}(\Omega)}^{2}},
$$

for some independent constant $B_{0}>0$, then the fully discrete version of the a priori bound (4.27) is true, i.e. replace all pertinent variables in (4.27) by their discrete counterparts. Moreover, if $\mathbf{V}_{h}^{0} \equiv \mathbf{0}$ on $\Omega_{h}$, then the fully discrete version of the conservation law (4.35) is also true.

We emphasize that the time step $\Delta t$ does not depend on the mesh size $h$ to guarantee stability or the conservation law; it only depends on $\left\|\mathbf{V}_{h}^{i+1}\right\|_{W^{1, \infty}(\Omega)}$ (see Remark 4.2).

REMARK 5.8 (Reason For The Lagrangian Update) Using a Lagrangian approach to update the temperatures (5.3) avoids having to compute the intersection of the mesh from one time step to the next (i.e. the $L^{2}$ projections (5.12) are only computed on the previous domains $\Omega_{1, h}^{i-1}, \Omega_{\mathrm{s}, h}^{i-1}$ ). The alternative would have been to compute the $L^{2}$ projection (for $j=1, \mathrm{~s}$ ) of $u_{j, h}^{i}$ from $\Omega_{j, h}^{i-1}$ to $\Omega_{j, h}^{i}$, which would require computing the intersection of the meshes representing $\Omega_{j, h}^{i-1}$ and $\Omega_{j, h}^{i}$. 


\subsection{Specific realization}

The particulars of our implementation are as follows. Let $\tau_{h}$ denote a quasi-uniform, shape regular triangulation of $\overline{\Omega_{h}}=\overline{\Omega_{1, h}} \cup \overline{\Omega_{\mathrm{s}, h}}$ consisting of affine tetrahedra $T$ of maximum size $h \equiv h_{T}$ [10]. We choose the finite element spaces in the bulk to be $\mathbb{V}_{1, h}=\mathrm{BDM}_{1} \subset H\left(\mathrm{div}, \Omega_{1, h}\right)$, $\mathbb{V}_{\mathrm{s}, h}=\mathrm{BDM}_{1} \subset H\left(\operatorname{div}, \Omega_{\mathrm{s}, h}\right)$, i.e. the lowest order Brezzi-Douglas-Marini space of piecewise linear vector functions $[7,26]$, and $\mathbb{Q}_{1, h}, \mathbb{Q}_{\mathrm{s}, h}$ to be the set of piecewise constants.

Next, assume that $\Gamma_{h}$ is represented by a conforming set of faces $\mathcal{F}_{h}$ in the triangulation $\mathcal{J}_{h}$, i.e. $\mathcal{F}_{h}$ is the surface triangulation obtained by restricting $\mathcal{J}_{h}$ to $\Gamma_{h}$. Then choose $\mathbb{M}_{h}$ to be the space of continuous piecewise linear functions over $\mathcal{F}_{h}$ and each of the three components of the space $\mathbb{Y}_{h}$ to be continuous piecewise linear functions over $\mathcal{F}_{h}$. Recalling Remark 5.2, we choose $\mathbb{L}_{h}$ to be the space of continuous piecewise linear functions over $\Omega_{h}$.

REMARK 5.9 (Choice Of Finite Element Spaces) It is well-known that these spaces satisfy the assumptions in Section 5.2. Indeed, it is possible to enforce zero boundary values point-wise with $\mathrm{BDM}_{1}$. If different spaces were chosen that did not allow this, then one needs a reasonable compatibility condition between $\stackrel{\ominus}{V}_{1, h}, \stackrel{\ominus}{V}_{s, h}$ and $\mathbb{M}_{h}$ in order to prove Lemma 5.6.

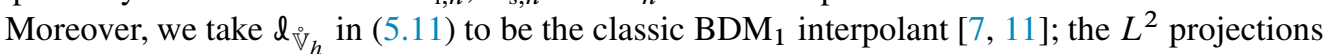
$\Pi_{\mathbb{Q}_{1, h}}, \Pi_{\mathbb{Q}_{\mathrm{s}, h}}$ are standard [10]. Note that this allows (5.3) to be computed locally (i.e. element-byelement).

\section{Numerical results}

We present two dimensional simulations to illustrate our method (2-D for simplicity). All simulations were implemented in the package FELICITY [62]. The linear systems are solved by MATLAB's “backslash" command. Alternatively, one can use an iterative procedure such as Uzawa's algorithm; see [22, Section 7] for an example in a related problem.

For all simulations, the Dirichlet boundary is the entire outer boundary, i.e. $\partial_{\mathrm{D}} \Omega \equiv \partial \Omega$ with $u_{\mathrm{D}}=-0.5$. The initial temperature is $u_{\mathrm{s}}^{0}:=0$ in $\Omega_{\mathrm{s}}$ and $u_{1}^{0}$ is a smooth function between 0 and -0.5 in $\Omega_{1}$. For updating the temperatures, we initialized $\mathbf{V}^{0}=\mathbf{0}$. We verified the conservation law by computing the left-hand-side of (4.35). The error was less than $10^{-3}$, which is consistent with the $O(\Delta t)$ error estimate in Theorem 4.16. During the course of a simulation, the mesh topology was regenerated between three and five times which did not impact the computational time.

Error estimates for the spatial discretization will be discussed in a future publication.

\subsection{Isotropic surface energy}

The model in Section 2 assumes the surface tension coefficient $\widehat{\alpha}$ is constant (isotropic). In Figure 2, we show a simulation of our method with a non-trivial initial shape. Also see Figure 1 for another example with a different initial shape. 

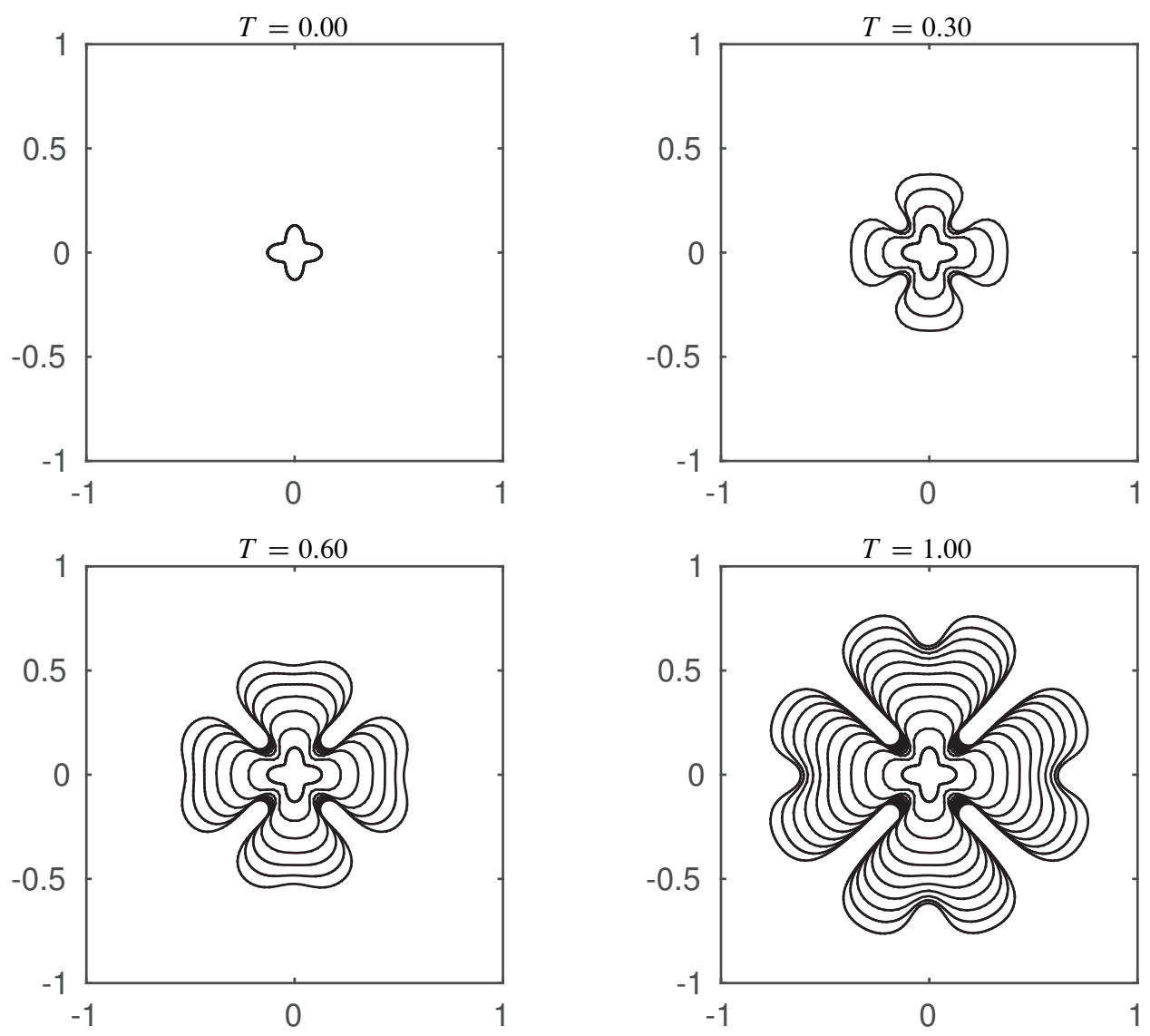

FIG.2: Simulation with isotropic surface tension. Several time-lapses are shown to illustrate the evolution with initial interface having a "clover" shape.

\subsection{Anisotropic surface energy}

The model can be generalized to have an anisotropic surface tension coefficient, i.e. $\widehat{\alpha} \equiv \widehat{\alpha}(\boldsymbol{\nu})$. In particular, we consider anisotropies of the form:

$$
\widehat{\alpha}=\widehat{\alpha}(\nu):=\widehat{\alpha}_{0} \sum_{j=1}^{K}\left(\nu^{T} G_{j} \nu\right)^{1 / 2},
$$

where $\widehat{\alpha}_{0}=0.0005$ is a material constant, $K$ is the number of anisotropies, and $G_{j}$ is a symmetric positive definite matrix in $\mathbb{R}^{d \times d}$. We consider a class of matrices that have the structure $G_{j}=R_{j}^{T} D_{j} R_{j}$, where $R_{j}$ is a rotation matrix that determines the "directions" of the anisotropy, and $D_{j}$ is a diagonal matrix consisting of ones and small numbers, which controls the strength of the anisotropy. For our simulations, we set $\widehat{\beta}=\widehat{\beta}_{0} \widehat{\alpha}(\nu)$, although this is not required. Note that isotropic surface tension is modeled by this as well with $K=1$ and $G_{1}=I_{2 \times 2}$ so that $\widehat{\alpha}(\boldsymbol{\nu})=\widehat{\alpha}_{0}$. 

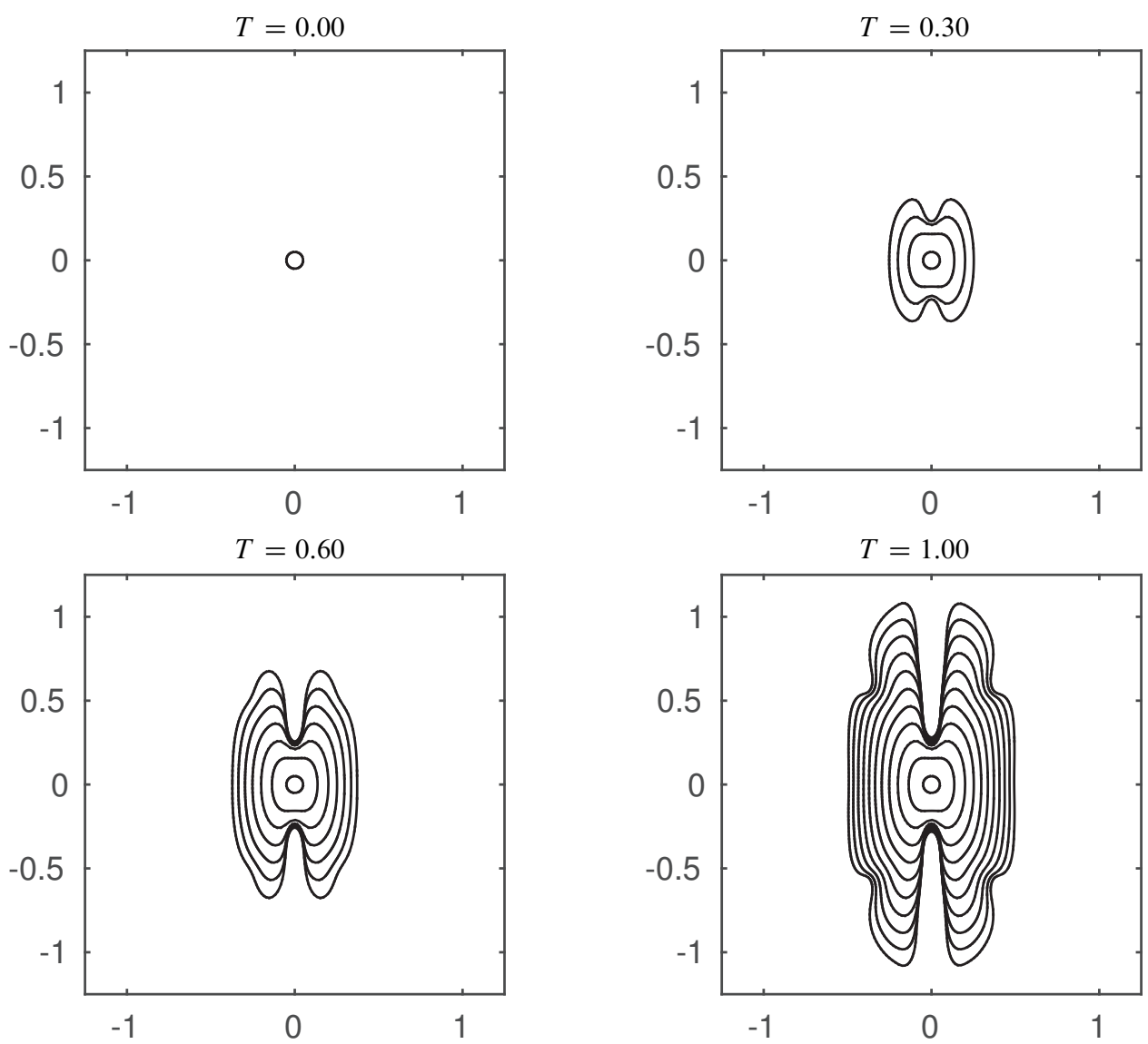

FIG. 3: Simulation with anisotropic surface tension. Several time-lapses are shown to illustrate the evolution with initial interface shape being a circle. A one-fold anisotropy is used which breaks the initial radial symmetry.

With the above, we can derive the modified form of (4.6) by standard shape differentiation $[16,32,56]$. Indeed,

$$
\frac{d}{d t} \int_{\Gamma(t)} \widehat{\alpha}(\boldsymbol{\nu})=\int_{\Gamma(t)} \widehat{\alpha}(\boldsymbol{\nu}) \nabla_{\Gamma} \mathbf{X}: \nabla_{\Gamma} \mathbf{V}-\int_{\Gamma(t)} \boldsymbol{\nu}\left[\widehat{\alpha}^{\prime}(\boldsymbol{\nu})\right]^{T}: \nabla_{\Gamma} \mathbf{V}
$$

where $\mathbf{V}$ is the velocity of $\Gamma$, and for $\mathbf{p} \in \mathbb{R}^{d}, \widehat{\alpha}^{\prime}(\mathbf{p})$ is the gradient of $\widehat{\alpha}$ with respect to $\mathbf{p}$. We now obtain a semi-discrete formulation for the anisotropic case by combining (4.5), (4.6), and (6.2):

$$
\begin{aligned}
\left(\widehat{\beta}^{-1}\left(\boldsymbol{\nu}^{i}\right) \mathbf{V}^{i+1} \cdot \boldsymbol{\nu}^{i}, \mathbf{Y}\right. & \left.\cdot \boldsymbol{\nu}^{i}\right)_{\Gamma^{i}}+\Delta t\left(\widehat{\alpha}\left(\boldsymbol{\nu}^{i}\right) \nabla_{\Gamma^{i}} \mathbf{V}^{i+1}, \nabla_{\Gamma^{i}} \mathbf{Y}\right)_{\Gamma^{i}}+\widehat{S}\left(\mathbf{Y} \cdot \boldsymbol{\nu}^{i}, \lambda^{i+1}\right)_{\Gamma^{i}} \\
& =-\left(\widehat{\alpha}\left(\boldsymbol{\nu}^{i}\right) \nabla_{\Gamma^{i}} \mathbf{X}^{i}, \nabla_{\Gamma^{i}} \mathbf{Y}\right)_{\Gamma^{i}}+\left(\boldsymbol{\nu}^{i}\left[\widehat{\alpha}^{\prime}\left(\boldsymbol{\nu}^{i}\right)\right]^{T}, \nabla_{\Gamma^{i}} \mathbf{Y}\right)_{\Gamma^{i}} \text { for all } \mathbf{Y} \in \mathbb{Y}^{i}
\end{aligned}
$$


The fully discrete formulation follows straightforwardly. This type of anisotropy is studied in [5] where they handle the anisotropic surface energy by defining the local finite element basis functions to capture the anisotropic energy. Their approach allows for obtaining an energy law, which can also be combined with our method. But (6.3) is easier to implement. In fact, it allows us to consider more general coefficients $\widehat{\alpha}(\boldsymbol{\nu})$ other than (6.1). The main drawback of (6.3) is it makes the numerical scheme slightly explicit, which puts a constraint on the time step. From our experience, we need $\Delta t \leqslant C h$ for some uniform constant $C$. Using the anisotropic approach in [5] would circumvent this.

In Figure 3, we present a simulation using (6.1) with $K=1$ (i.e. a one-fold anisotropy). Figure 4 shows a simulation with $K=3$ (i.e. a three-fold anisotropy).
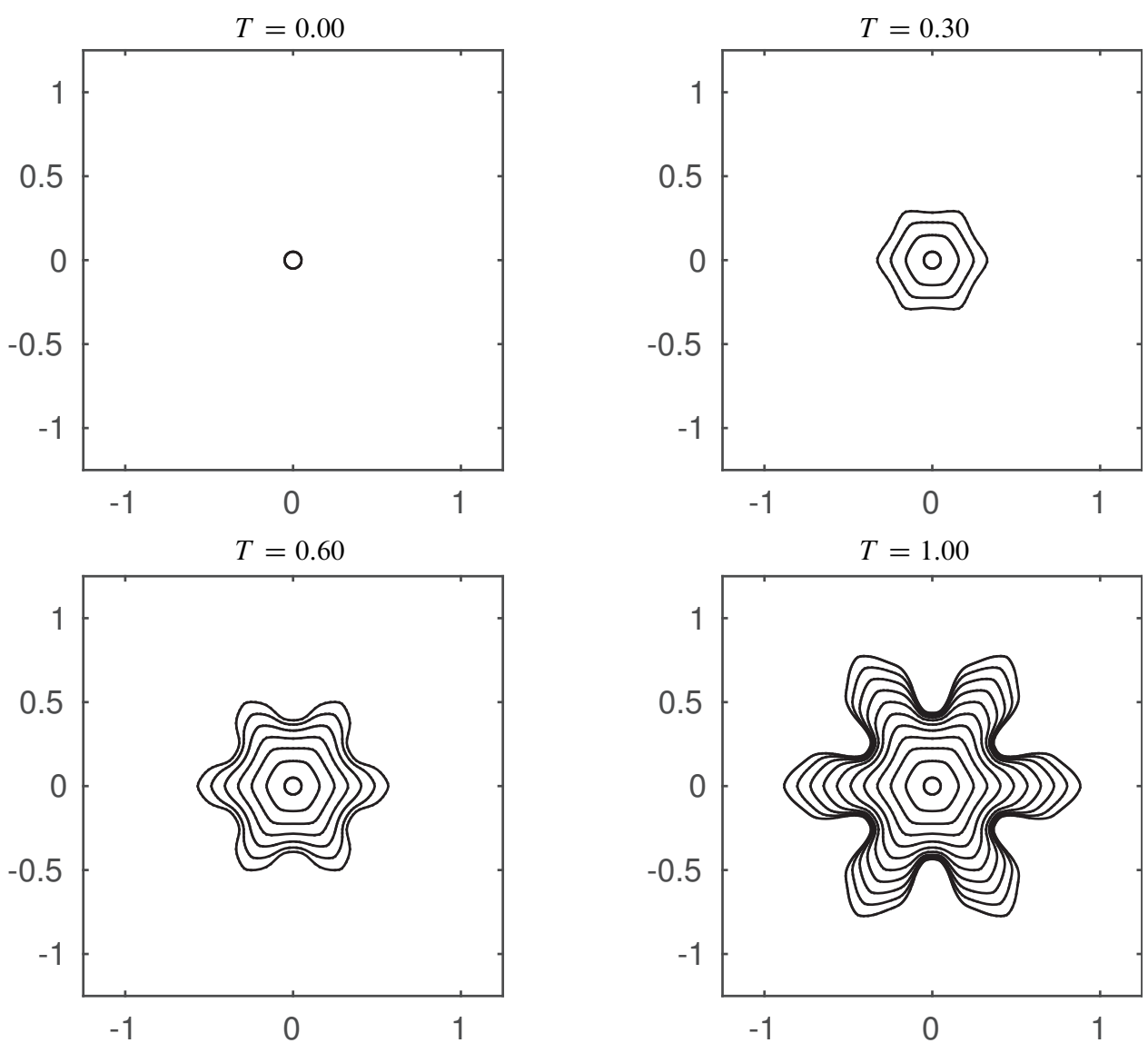

FIG.4: Simulation with anisotropic surface tension. Several time-lapses are shown to illustrate the evolution with initial interface shape being a circle. A three-fold anisotropy is used which breaks the initial radial symmetry. 


\section{Acknowledgments}

Davis acknowledges financial support by the National Science Foundation through the following grant: DMS-0739382. Walker acknowledges financial support by the National Science Foundation through the following grants: DMS-1115636, DMS-1418994. The research of the third author was partially supported by NSF DMS-1265579.

\section{REFERENCES}

1. Adams, R. A. \& Fournier, J. J. F., Sobolev Spaces, 2nd ed., vol. 140 of Pure and Applied Mathematics Series. Elsevier, 2003. Zbl1098.46001 MR2424078

2. Almgren, R., Variational algorithms and pattern formation in dendritic solidification. Journal of Computational Physics 1062 (1993), 337 - 354. Zb11098. 46001 MR2424078

3. B ̈̈NSCH, E., Finite element discretization of the navier-stokes equations with a free capillary surface. Numerische Mathematik 88 (2001), 203-235. Zbl0985. 35060 MR1826211

4. BÄNSCh, E., PAUl, J. \& SChMidt, A., An ale finite element method for a coupled stefan problem and Navier-Stokes equations with free capillary surface. International Journal for Numerical Methods in Fluids 71 (2013), 1282-1296. Zb10985. 35060 MR1826211

5. BARrett, J. W., GARCKe, H. \& NÜrnberg, R., On stable parametric finite element methods for the stefan problem and the mullins-sekerka problem with applications to dendritic growth. Journal of Computational Physics 229 (Sept. 2010), 6270-6299. Zb10985 . 35060 MR1826211

6. Boettinger, W. J., Warren, J. A., Beckermann, C. \& Karma, A., Phase-field simulation of solidification. Annual Review of Materials Research 32 (Aug 2002), 163-194.

7. Boffi, D., Brezzi, F. \& Fortin, M., Mixed Finite Element Methods and Applications, vol. 44 of Springer Series in Computational Mathematics. Springer-Verlag, New York, NY, 2013. Zb11277. 65092 MR3097958

8. Boffi, D. \& Gastaldi, L., Analysis of finite element approximation of evolution problems in mixed form. SIAM Journal on Numerical Analysis 42 (2005), 1502-1526. Zbl1080 65089 MR2114288

9. Braess, D., Finite Elements: Theory, Fast Solvers, and Applications in Solid Mechanics, 2nd ed. Cambridge University Press, 2001.

10. Brenner, S. C. \& SCOTT, L. R., The Mathematical Theory of Finite Element Methods, 3rd ed., vol. 15 of Texts in Applied Mathematics. Springer, New York, NY, 2008. Zb11135.65042 MR2373954

11. Brezzi, F. \& Fortin, M., Mixed and Hybrid Finite Element Methods. Springer-Verlag, New York, NY, 1991. Zb10788.73002 MR1115205

12. Chen, S., Merriman, B., Osher, S. \& Smereka, P., A simple level set method for solving stefan problems. Journal of Computational Physics 135 (1997), 8-29. Zbl0889.65133 MR1461705

13. Chen, X. \& Reitich, F., Local existence and uniqueness of solutions of the stefan problem with surface tension and kinetic undercooling. Journal of Mathematical Analysis and Applications 164 (1992), 350362. Zb10761.35113 MR1151039

14. Cheng, C. H. A., Coutand, D. \& Shkoller, S., Navier-stokes equations interacting with a nonlinear elastic biofluid shell. SIAM Journal of Mathematical Analysis 39 (2007), 742-800. Zb11138.74022 MR2349865

15. Davis, S. H., Theory of Solidification. Cambridge Monographs on Mechanics. Cambridge University Press, 2001. Zbl0991.76002 MR1868285

16. Delfour, M. C. \& ZolÉsio, J.-P., Shapes and Geometries: Analysis, Differential Calculus, and Optimization, 2nd ed., vol. 4 of Advances in Design and Control. SIAM, 2011. Zbl1002.49029 MR1855817

17. Do Carmo, M. P., Differential Geometry of Curves and Surfaces. Prentice Hall, Upper Saddle River, New Jersey, 1976. Zb10326.53001 MR0394451 
18. Duchon, J. \& Robert, R., Évolution d'une interface par capillarité et diffusion de volume. i. existence locale en temps. Annales de l'institut Henri Poincaré (C) Analyse non linéaire 1 (1984), 361-378. Zb10572.35051 MR0779874

19. DZIUK, G., An algorithm for evolutionary surfaces. Numerische Mathematik 58 (1990), 603-611. Zb10714.65092 MR1083523

20. Escher, J., Simonett, G., Alikakos, N. \& Bates, P., Classical solutions for hele-shaw models with surface tension. Adv. Differential Equations 2 (1997), 619-642.

21. Evans, L. C., Partial Differential Equations. American Mathematical Society, Providence, Rhode Island, 1998. Zbl0902.35002 MR1625845

22. FAlK, R. S. \& WALKer, S. W., A mixed finite element method for EWOD that directly computes the position of the moving interface. SIAM Journal on Numerical Analysis 51 (Mar 2013), 1016-1040. Zbl1268.76063 MR3035483

23. FrIED, M., A level set based finite element algorithm for the simulation of dendritic growth. Computing and Visualization in Science 7 (2004), 97-110. Zbl1120.80310 MR2088785

24. Friedman, A. \& Reitich, F., The stefan problem with small surface tension. Trans. Amer. Math. Soc. 328 (1991), 465-515. Zbl0744.35068 MR1040260

25. GARCKE, H. \& SCHAUbECK, S., Existence of weak solutions for the stefan problem with anisotropic Gibbs-Thomson law. Adv. Math. Sci. Appl. 21 (2011), 255-283. Zbl1235. 35286 MR2883883

26. Gatica, G. N., A Simple Introduction to the Mixed Finite Element Method: Theory and Applications. SpringerBriefs in Mathematics. Springer, Jan 2014. Zbl1293.65152 MR3157367

27. Gerbeau, J.-F. \& LeliÈvre, T. Generalized navier boundary condition and geometric conservation law for surface tension. Computer Methods in Applied Mechanics and Engineering 198 (2009), 644-656. Zbl1229.76037 MR2498521

28. Gerbeau, J.-F., Lelièvre, T. \& Bris, C. L., Simulations of MHD flows with moving interfaces. Journal of Computational Physics 184 (2003), 163-191. Zbl1118.76359 MR1961974

29. Gurtin, M. E., Multiphase thermomechanics with interfacial structure 1. heat conduction and the capillary balance law. Archive for Rational Mechanics and Analysis 104 (1988), 195-221. Zbl0723. 73016 MR1017288

30. Gurtin, M. E., Thermomechanics of Evolving Phase Boundaries in the Plane. Oxford Mathematical Monographs. Oxford Science Publication, 1993. Zb10787.73004 MR1402243

31. Hadzic, M. \& ShKoller, S., Global stability and decay for the classical stefan problem. Commun. Pure Appl. Math. 68 (2015), 689-757. Zbl 06430102 MR3393322

32. Haslinger, J. \& MäKinen, R. A. E., Introduction to Shape Optimization: Theory, Approximation, and Computation, vol. 7 of Advances in Design and Control. SIAM, 2003. Zb11020.74001 MR1969772

33. Holzapfel, G. A., Nonlinear Solid Mechanics: A Continuum Approach For Engineering. John Wiley \& Sons, Inc., 2000. Zbl0980.74001 MR1827472

34. Juric, D. \& Tryggvason, G., A front-tracking method for dendritic solidification. Journal of Computational Physics 123 (1996), 127-148. Zb10843.65093 MR1370379

35. Kobayashi, R., A numerical approach to three-dimensional dendritic solidification. Experimental Mathematics 3 (1994), 59-81. Zb10811.65126 MR1302819

36. Kraus, C., The degenerate and non-degenerate stefan problem with inhomogeneous and anisotropic Gibbs-Thomson law. European Journal of Applied Mathematics 22 (2011), 393-422. Zb11258.35211 MR2834012

37. KreysZIG, E., Differential Geometry. Dover, 1991. MR1118149

38. LANGeR, J. S., Instabilities and pattern formation in crystal growth. Rev. Mod. Phys. 52 (Jan 1980), 1-28.

39. LuCKhaus, S., Solutions for the two-phase stefan problem with the gibbs-thomson law for the melting temperature. In Fundamental Contributions to the Continuum Theory of Evolving Phase Interfaces in Solids, J. M. Ball, D. Kinderlehrer, P. Podio-Guidugli,, \& M. Slemrod, Eds. Springer Berlin Heidelberg, 1999, 317-327. MR1770902 
40. Luckhaus, S. \& Sturzenhecker, T., Implicit time discretization for the mean curvature flow equation. Calculus of Variations and Partial Differential Equations 3 (1995), 253-271. Zb10821. 35003 MR1386964

41. Mullins, W. W. \& Sekerka, R. F., Morphological stability of a particle growing by diffusion or heat flow. Journal of Applied Physics 34 (1963), 323-329.

42. Mullins, W. W. \& Sekerka, R. F., Stability of a planar interface during solidification of a dilute binary alloy. Journal of Applied Physics 35 (1964), 444-451.

43. Nochetto, R. H., Salgado, A. J. \& Walker, S. W., A diffuse interface model for electrowettng with moving contact lines. Mathematical Models and Methods in Applied Sciences 24 (2014), 67-111.

44. Osher, S. \& FedKiw, R., Level Set Methods and Dynamic Implicit Surfaces. Springer-Verlag, New York, NY, 2003. Zbl1026.76001 MR1939127

45. PrüsS, J., SAAl, J. \& Simonett, G., Existence of analytic solutions for the classical stefan problem. Mathematische Annalen 338 (2007), 703-755. Zb11130.35136 MR2317935

46. PRÜss, J. \& Simonett, G., Stability of equilibria for the stefan problem with surface tension. SIAM Journal on Mathematical Analysis 40 (2008), 675-698. Zbl1157.35502 MR2438781

47. Prüss, J., Simonett, G. \& Wilke, M., On thermodynamically consistent stefan problems with variable surface energy. submitted, arXiv (2011).

48. Prüss, J., Simonett, G. \& ZACHER, R., Qualitative behavior of solutions for thermodynamically consistent stefan problems with surface tension. Archive for Rational Mechanics and Analysis 207 (2013), 611-667. Zbl1269.80004 MR3005325

49. RÖGER, M., Existence of weak solutions for the mullins-sekerka flow. SIAM Journal on Mathematical Analysis 37 (2005), 291-301. Zb11088. 49031 MR2176933

50. Roosen, A. R. \& TAYlor, J. E., Modeling crystal growth in a diffusion field using fully faceted interfaces. Journal of Computational Physics 114 (1994), 113-128. Zbl0805.65128 MR1286190

51. SCHMidT, A., Computation of three dimensional dendrites with finite elements. Journal of Computational Physics 125 (1996), 293-312. Zbl0844.65096

52. Schmidt, A., Approximation of crystalline dendrite growth in two space dimensions. Acta Math. Univ. Comenianae 67 (1998), 57-68. Zb10930.65107 MR1660815

53. Schmidt, A. \& Siebert, K. G., Design of Adaptive Finite Element Software: The Finite Element Toolbox ALBERTA, 1st ed. Springer, Heidelberg, Germany, 2005. Zbl1068.65138 MR2127659

54. Sethian, S. A., Level Set Methods and Fast Marching Methods, 2nd Edition. Cambridge University Press, New York, NY, 1999. Zbl0973.76003 MR1700751

55. Singer-Loginova, I. \& Singer, H. M., The phase field technique for modeling multiphase materials. Reports on Progress in Physics 71 (2008), 106501.

56. Sokolowski, J. \& ZolÉsio, J.-P., Introduction to Shape Optimization. Springer Series in Computational Mathematics. Springer-Verlag, 1992. Zb10761.73003 MR1215733

57. TARTAR, L., An Introduction to Sobolev Spaces and Interpolation Spaces, vol. 3 of Lecture Notes of the Unione Matematica Italiana. Springer, 2007. Zb11126. 46001 MR2328004

58. Temam, R. M. \& Miranville, A. M., Mathematical Modeling in Continuum Mechanics, 2nd ed. Cambridge University Press, 2005. MR2169020

59. Visintin, A., Models of Phase Transitions, vol. 28 of Progress in Nonlinear Differential Equations. Birkhäuser, Boston, 1996. Zbl0882. 35004 MR1423808

60. Volkov, O. \& Protas, B., An inverse model for a free-boundary problem with a contact line: Steady case. Journal of Computational Physics 228 (July 2009), 4893-4910. Zbl1166. 76056 MR2533162

61. Voronkov, V. V., Conditions for formation of mosaic structure on a crystallization front. Sov. Phys. Solid State 6 (1965), 2378-2381.

62. WALKER, S. W., FELICITY: Finite ELement Implementation and Computational Interface Tool for You. http://www . mathworks . com/matlabcentral/fileexchange/31141-felicity. 
63. WALKER, S. W., Tetrahedralization of isosurfaces with guaranteed-quality by edge rearrangement (TIGER). SIAM Journal on Scientific Computing 35 (2013), A294-A326. Zb11264. 65026 MR3033050

64. WALker, S. W., A mixed formulation of a sharp interface model of stokes flow with moving contact lines. ESAIM: Mathematical Modelling and Numerical Analysis 48 (July 2014), 969-1009. Zbl1299. 76064 MR3264343

65. Walker, S. W., Bonito, A. \& Nochetto, R. H., Mixed finite element method for electrowetting on dielectric with contact line pinning. Interfaces and Free Boundaries 12 (March 2010), 85-119. Zbl1189. 78056 MR2595379 